\title{
COUNTING SUBGRAPHS IN QUASI-RANDOM 4-UNIFORM HYPERGRAPHS
}

\author{
VOJTĚCH RÖDL AND JOZEF SKOKAN
}

\begin{abstract}
A bipartite graph $G=\left(V_{1} \cup V_{2}, E\right)$ is $(\delta, d)$-regular if

$$
\left|d-d\left(V_{1}^{\prime}, V_{2}^{\prime}\right)\right|<\delta
$$

whenever $V_{i}^{\prime} \subset V_{i},\left|V_{i}^{\prime}\right| \geq \delta\left|V_{i}\right|, i=1,2$. Here, $d\left(V_{1}^{\prime}, V_{2}^{\prime}\right)=e\left(V_{1}^{\prime}, V_{2}^{\prime}\right) /\left|V_{1}^{\prime}\right|\left|V_{2}^{\prime}\right|$ stands for the density of the pair $\left(V_{1}^{\prime}, V_{2}^{\prime}\right)$.

An easy counting argument shows that if $G=\left(V_{1} \cup V_{2} \cup V_{3}, E\right)$ is a 3-partite graph whose restrictions on $V_{1} \cup V_{2}, V_{1} \cup V_{3}, V_{2} \cup V_{3}$ are $(\delta, d)$ regular, then $G$ contains $\left(d^{3} \pm f(\delta)\right)\left|V_{1}\right|\left|V_{2}\right|\left|V_{3}\right|$ copies of $K_{3}$. This fact and its various extensions are the key ingredients in most applications of Szemerédi's Regularity Lemma.

To derive a similar results for $r$-uniform hypergraphs, $r>2$, is a harder problem. In 1994, Frankl and Rödl developed a regularity lemma and counting argument for 3-uniform hypergraphs. In this paper, we exploit their approach to develop a counting argument for 4-uniform hypergraphs.
\end{abstract}

\section{INTRODUCTION}

While proving his well-known Density Theorem [Sze75], E. Szemerédi discovered an auxiliary lemma which he soon transformed into a powerful tool in extremal graph theory. This result, named the Regularity lemma [Sze78], states that all sufficiently large graphs can be approximated by "random-like" graphs. This feature is especially useful in situations when the problem in question is easier to prove for random graphs.

In particular, one such situation is the counting copies of a given small graph in another graph. Although this problem is very hard in general, there is a simple argument (called the Counting Lemma) which counts these copies in the approximation produced by the Regularity Lemma. Since the Counting Lemmas as well as the Regularity Lemma have had numerous applications (see [KS96, KSSS02] for survey), a natural question arises whether they can be generalized to hypergraphs.

Date: October 12, 2004.

The first author was partially supported by NSF grants DMS-0071261, DMS-0300529 and INT-0072064.

The second author was partially supported by NSF grants INT-0072064 and INT0305793 and NSA grant H98230-04-1-0035. 
Chung [Chu91] and Frankl and Rödl [FR92] considered regularity lemmas for hypergraphs. Yet these regularity lemmas failed to produce "randomlike" approximations in which one could count copies of given small hypergraphs. This is perhaps the main reason why the regularity lemmas from [FR92, Chu91] did not have many applications. As an attempt to improve the situation, Frankl and Rödl considered a strengthening of the approach from [FR92] that for 3-uniform hypergraph enables to find a copy of the complete 3 -uniform hypergraph on four vertices $K_{4}^{(3)}$ in a " $\delta$-regular" 3 -uniform hypergraph. Their result was announced already in [Ro91], but it has been published only recently [FR02]. Frankl and Rödl's result was later generalized to the counting of arbitrary small 3-uniform hypergraphs by Nagle and Rödl [NR03]. These counting lemmas have already had several applications (see e.g. [KNR03, NR02, RR98]).

The purpose of this paper is to develop a further extension of the counting lemma from [FR02] and generalize it to 4-uniform hypergraphs.

Let us mention that our result, together with the regularity lemma for $k$-uniform hypergraphs recently developed in [RS04], can be used in several applications. In particular, it gives an answer to the problem of Székely (cf. [Ma02], page 227) and confirms Conjecture 1.5 in [FR02] for $k=5$. Since the argument for counting in 4-uniform hypergraphs presented in this paper is technical and long, these applications will appear in a separate note [RS04a].

1.1. Notation and basic definitions. We start with some definitions. For a set $V$ and an integer $k \geq 2$, let $[V]^{k}$ denote the system of all $k$-element subsets of $V$. An ordered pair $\mathcal{G}=(V(\mathcal{G}), E(\mathcal{G}))=(V, E)$, where $E=E(\mathcal{G})$ is a subset of $[V]^{k}$, is called a $k$-uniform hypergraph. If $k=2$, we have a graph.

Let $V=V_{1} \cup \cdots \cup V_{s}$ be a partition, we say that a set $e \subset V$ is crossing if $\left|e \cap V_{j}\right| \leq 1$ for all $j=1,2, \ldots, s$. Furthermore, a hypergraph $\mathcal{G}=$ $\left(V_{1} \cup \cdots \cup V_{s}, E\right)$ is said to be $s$-partite if its all edges are crossing. We shall also denote by $K_{s}^{(k)}\left(V_{1}, \ldots, V_{s}\right)$ the complete $k$-uniform $s$-partite hypergraph with partition $V_{1} \cup \cdots \cup V_{s}$.

This paper deals with $s$-partite $k$-uniform hypergraphs, which we call $(s, k)$-cylinders.

Definition 1.1. Let $s \geq k \geq 1$ be two integers. We define an $(s, k)$-cylinder $\mathcal{G}$ as follows.

For $k=1, \mathcal{G}$ is a partition $V(\mathcal{G})=V_{1} \cup \ldots \cup V_{s}$. For $k>1, \mathcal{G}$ is any $s$-partite $k$-uniform hypergraph.

If there is no danger of confusion, we shall identify $(s, k)$-cylinders with their edge sets.

Definition 1.2. Let $k=1$ and let $\mathcal{G}, \mathcal{G}^{\prime}$ be two $(s, 1)$-cylinders, $V(\mathcal{G})=$ $V_{1} \cup \ldots \cup V_{s}$ and $V\left(\mathcal{G}^{\prime}\right)=V_{1}^{\prime} \cup \ldots \cup V_{s}^{\prime}$. We say that $\mathcal{G}^{\prime}$ is a subcylinder of $\mathcal{G}$ if $V_{i}^{\prime} \subset V_{i}$ for all $i=1,2, \ldots, s$. 
For $k>1$ and two $(s, k)$-cylinders $\mathcal{G}, \mathcal{G}^{\prime}$, we say that $\mathcal{G}^{\prime}$ is a subcylinder of $\mathcal{G}$ if $E\left(\mathcal{G}^{\prime}\right) \subset E(\mathcal{G})$. Moreover, $\mathcal{G}^{\prime}$ is an induced subcylinder of $\mathcal{G}$, and we write $\mathcal{G}^{\prime}=\mathcal{G}\left[V\left(\mathcal{G}^{\prime}\right)\right]$, if $E\left(\mathcal{G}^{\prime}\right)=E(\mathcal{G}) \cap\left[V\left(\mathcal{G}^{\prime}\right)\right]^{k}$.

If $s=k+1$, we will often write an $(s, k)$-cylinder $\mathcal{G}$ as $\mathcal{G}=\bigcup_{i=1}^{s} \partial_{i} \mathcal{G}$, where $\partial_{i} \mathcal{G}$ is the subcylinder of $\mathcal{G}$ induced on $\bigcup_{j \neq i} V_{j}$.

A subcylinder $\mathcal{G}^{\prime}=\left(V^{\prime}, E^{\prime}\right)$ of $\mathcal{G}$ is a clique in $\mathcal{G}$ if $E^{\prime}=\left[V^{\prime}\right]^{k}$.

Definition 1.3. For an $(s, 1)$-cylinder $\mathcal{G}=V_{1} \cup \cdots \cup V_{s}$ and $1 \leq j \leq s$, we define $\mathcal{K}_{j}(\mathcal{G})=K_{s}^{(j)}\left(V_{1}, \ldots, V_{s}\right)$. For an $(s, k)$-cylinder $\mathcal{G}$, where $k>1$, we shall denote by $\mathcal{K}_{j}(\mathcal{G}), k \leq j \leq s$, the $j$-uniform hypergraph whose edges are precisely those $j$-element subsets of $V(\mathcal{G})$ that span cliques of order $j$ in $\mathcal{G}$.

Clearly, for $k>1$, the quantity $\left|\mathcal{K}_{j}(\mathcal{G})\right|$ counts the total number of cliques of order $j$ in $\mathcal{G}$. We will often face a situation when we need to describe that one cylinder 'lies on' another cylinder. To this end, we define the term underlying cylinder.

Definition 1.4. Let $\mathcal{G}$ be an $(s, k-1)$-cylinder and $\mathcal{H}$ be an $(s, k)$-cylinder with the same s-partition. We say that $\mathcal{G}$ underlies $\mathcal{H}$ if $\mathcal{H} \subset \mathcal{K}_{k}(\mathcal{G})$.

Through this paper, we will work with a series of underlying cylinders. To accommodate this situation, we introduce the notion of complex.

Definition 1.5. Let $s$ and $k, s \geq k \geq 2$, be two integers. An $(s, k)$-complex $\mathcal{H}$ is a system of cylinders $\left\{\mathcal{H}^{(i)}\right\}_{i=1}^{k}$ such that

(a) $\mathcal{H}^{(1)}$ is an $(s, 1)$-cylinder $V_{1} \cup \cdots \cup V_{s}$,

(b) for every $i \in[k-1], \mathcal{H}^{(i)}$ underlies $\mathcal{H}^{(i+1)}$, i.e. $\mathcal{H}^{(i+1)} \subset \mathcal{K}_{i+1}\left(\mathcal{H}^{(i)}\right)$.

1.2. Regularity for graphs. Before we state the Regularity Lemma, we must introduce the concept of regular pairs.

Definition 1.6 ([Sze78]). Let $G=(V, E)$ be a graph and $\delta$ be a positive real number, $0<\delta \leq 1$. We say that a pair $(A, B)$ of two disjoint subsets of $V$ is $\delta$-regular if

$$
\left|d\left(A^{\prime}, B^{\prime}\right)-d(A, B)\right|<\delta
$$

for any two subsets $A^{\prime} \subset A, B^{\prime} \subset B,\left|A^{\prime}\right| \geq \delta|A|,\left|B^{\prime}\right| \geq \delta|B|$. Here, $d(A, B)=|E(A, B)| /(|A||B|)$ stands for the density of the pair $(A, B)$.

This definition states that a regular pair has uniformly distributed edges. The Regularity Lemma of Szemerédi [Sze78] enables us to partition the vertex set $V(G)$ of a graph $G$ into $t+1$ sets $V_{0} \cup V_{1} \cup \ldots \cup V_{t}$ in such a way that most of the pairs $\left(V_{i}, V_{j}\right)$ satisfy Definition 1.6. The precise statement is following.

Theorem 1.7 (Regularity Lemma [Sze78]). For every $\delta>0$ and $t_{0} \in \mathbb{N}$ there exist two integers $N_{0}=N_{0}\left(\delta, t_{0}\right)$ and $T_{0}=T_{0}\left(\delta, t_{0}\right)$ with the following property: for every graph $G$ with $n \geq N_{0}$ vertices there is a partition of the 
vertex set into $t+1$ classes

$$
V=V_{0} \cup V_{1} \cup \ldots \cup V_{t}
$$

such that

(i) $t_{0} \leq t \leq T_{0}$,

(ii) $\left|V_{0}\right| \leq \delta n,\left|V_{1}\right|=\ldots=\left|V_{t}\right|$, and

(iii) all but at most $\delta\left(\begin{array}{c}t \\ 2\end{array}\right)$ pairs $\left(V_{i}, V_{j}\right), 1 \leq i<j \leq t$, are $\delta$-regular.

Moreover, this lemma is sufficiently strong to ensure the existence of various small subgraphs in $G$. The easiest case, when we count copies of $K_{3}$, is summarized in the next fact.

Fact 1.8. Let $G$ be a graph and $V_{i}, V_{j}, V_{k}$ be mutually disjoint subsets of $V(G)$. If all $\left(V_{i}, V_{j}\right),\left(V_{i}, V_{k}\right)$, and $\left(V_{j}, V_{k}\right)$ are $\delta$-regular pairs in $G$ with density $d$ and $2 \delta<d$, then

$$
(1-2 \delta)(d-\delta)^{3}\left|V_{i}\right|\left|V_{j}\right|\left|V_{k}\right| \leq\left|\mathcal{K}_{3}\left(G \cap K\left(V_{i}, V_{j}, V_{k}\right)\right)\right| \leq\left[2 \delta+(d+\delta)^{3}\right]\left|V_{i}\right|\left|V_{j}\right|\left|V_{k}\right| .
$$

This fact and its extensions (cf. Fact 4.5) are a key to many applications of the Regularity Lemma (cf. [CRST83, KSS97, KS96, KSSS02]).

1.3. Regularity for hypergraphs. Now we define the notion of regularity for cylinders:

Definition 1.9. Let $\mathcal{G}$ be a $(k, k-1)$-cylinder underlying a $(k, k)$-cylinder $\mathcal{H}$. We say that $\mathcal{H}$ is $(\delta, d)$-regular with respect to $\mathcal{G}$ if the following condition is satisfied: whenever $\mathcal{G}^{\prime} \subset \mathcal{G}$ is a $(k, k-1)$-cylinder such that

$$
\left|\mathcal{K}_{k}\left(\mathcal{G}^{\prime}\right)\right| \geq \delta\left|\mathcal{K}_{k}(\mathcal{G})\right|
$$

then

$$
\left|\mathcal{H} \cap \mathcal{K}_{k}\left(\mathcal{G}^{\prime}\right)\right|=(d \pm \delta)\left|\mathcal{K}_{k}\left(\mathcal{G}^{\prime}\right)\right|
$$

Here, $d \pm \delta$ stands for a number in the interval $(d-\delta, d+\delta)$. Note that for $k=2$, Definition 1.9 varies from Szemerédi's definition of a $\delta$-regular pair $\left(V_{1}, V_{2}\right)$ (cf. [Sze78]). This is because the $(2,1)$-cylinder $\mathcal{G}^{\prime}$ is a pair of sets $V_{i}^{\prime} \subset V_{i}, i=1,2$, and the condition $\left|\mathcal{K}_{k}\left(\mathcal{G}^{\prime}\right)\right| \geq \delta\left|\mathcal{K}_{k}(G)\right|$ translates to $\left|V_{1}^{\prime}\right|\left|V_{2}^{\prime}\right| \geq \delta\left|V_{1}\right|\left|V_{2}\right|$. However, it is easy to observe that:

- $(\delta, d)$-regularity implies $\delta^{1 / 2}$-regularity in the sense of Definition 1.6, and

- $\delta$-regularity in the above sense gives also $(\delta, d)$-regularity.

For $k>2$, the situation becomes more complicated and due to the quantification of constants in the hypergraph regularity lemma (Remark 4.6, [FR02]), Definition 1.9 is not strong enough to have the effect of Definition 1.6 in the case $k=2$. To overcome this problem, Frankl and Rödl introduced in [FR02] the concept of $(\delta, r)$-regularity. Here we present this concept in more general form. 
Definition 1.10. Let $r \in \mathbb{N}$ and $\mathcal{G}$ be a $(k, k-1)$-cylinder underlying a $(k, k)$-cylinder $\mathcal{H}$. We say that $\mathcal{H}$ is $(\delta, d, r)$-regular with respect to $\mathcal{G}$ if the following condition is satisfied: whenever $\mathcal{G}_{1}, \ldots, \mathcal{G}_{r} \subset \mathcal{G}$ are $(k, k-1)$ cylinders such that

$$
\left|\bigcup_{j=1}^{r} \mathcal{K}_{k}\left(\mathcal{G}_{j}\right)\right| \geq \delta\left|\mathcal{K}_{k}(\mathcal{G})\right|
$$

then

$$
\left|\mathcal{H} \cap \bigcup_{j=1}^{r} \mathcal{K}_{k}\left(\mathcal{G}_{j}\right)\right|=(d \pm \delta)\left|\bigcup_{j=1}^{r} \mathcal{K}_{k}\left(\mathcal{G}_{j}\right)\right| .
$$

We extend the above definition to the case of an $(s, k)$-cylinder $\mathcal{H}$.

Definition 1.11. Let $r \in \mathbb{N}$ and $\mathcal{G}$ be an $(s, k-1)$-cylinder underlying an $(s, k)$-cylinder $\mathcal{H}$. We say that $\mathcal{H}$ is $(\delta, d, r)$-regular with respect to $\mathcal{G}$ if $\mathcal{H}\left[\bigcup_{j \in I} V_{j}\right]$ is $(\delta, d, r)$-regular with respect to $\mathcal{G}\left[\bigcup_{j \in I} V_{j}\right]$ for all $I \in[s]^{k}$.

Now we are ready to introduce the concept of regularity for an $(s, k)$ complex $\mathcal{H}$.

Definition 1.12. Let $r \in \mathbb{N}$ and $\boldsymbol{d}=\left(d_{2}, \ldots, d_{k}\right)$ and $\boldsymbol{\delta}=\left(\delta_{2}, \ldots, \delta_{k}\right)$ be two vectors of positive real numbers such that $0<\delta_{i}<d_{i} \leq 1$ for all $i=2, \ldots k$. We say that an $(s, k)$-complex $\mathcal{H}$ is $(\boldsymbol{\delta}, \boldsymbol{d}, r)$-regular if

(a) $\mathcal{H}^{(2)}$ is $\left(\delta_{2}, d_{2}\right)$-regular with respect to $\mathcal{H}^{(1)}$, and

(b) $\mathcal{H}^{(i+1)}$ is $\left(\delta_{i+1}, d_{i+1}, r\right)$-regular with respect to $\mathcal{H}^{(i)}$ for every $i \in$ $[k-1] \backslash\{1\}$.

Frankl and Rödl proved the following theorem:

Theorem 1.13 ([FR02]). For any $\nu>0$ and any $d_{3} \in(0,1]$ there is a real number $\delta_{3}$ such that for any positive real number $d_{2} \in(0,1]$ there exist $\delta_{2}$ and $r, n_{0} \in \mathbb{N}$ such that if $\mathcal{H}=\left\{\mathcal{H}^{(1)}, \mathcal{H}^{(2)}, \mathcal{H}^{(3)}\right\}$ is a $(\boldsymbol{\delta}, \boldsymbol{d}, r)$-regular $(4,3)$ complex, where $\mathcal{H}^{(1)}=V_{1} \cup V_{2} \cup V_{3} \cup V_{4},\left|V_{1}\right|=\left|V_{2}\right|=\left|V_{3}\right|=\left|V_{4}\right|=n>n_{0}$, $\boldsymbol{d}=\left(d_{2}, d_{3}\right)$ and $\boldsymbol{\delta}=\left(\delta_{2}, \delta_{3}\right)$, then $\mathcal{H}^{(3)}$ contains $(1 \pm \nu) d_{3}^{\left(\begin{array}{c}4 \\ 3\end{array}\right)} d_{2}^{\left(\begin{array}{l}4 \\ 2\end{array}\right)} n^{4}$ copies of $K_{4}^{(3)}$.

This theorem plays the role of Fact 1.8. Indeed, it enables us to find copies of the complete 3 -uniform hypergraph on 4 vertices $K_{4}^{(3)}$ in 3-cylinders underlied by a regular sparse 2-cylinder. However, this theorem would be useless without an appropriate version of a regularity lemma for 3-uniform hypergraphs. Such a lemma was also introduced in [FR02]. Moreover, this result was extended by Nagle and Rödl in [NR03] who developed an argument for counting copies of the complete 3 -uniform hypergraph on $k$ vertices $K_{k}^{(3)}$.

Theorem 1.14 ([NR03]). For any integer $k \geq 4, \nu>0$ and any $d_{3} \in(0,1]$ there is a real number $\delta_{3}$ such that for any positive real number $d_{2} \in(0,1]$ 
there exist $\delta_{2}$ and $r, n_{0} \in \mathbb{N}$ such that if $\mathcal{H}=\left\{\mathcal{H}^{(1)}, \mathcal{H}^{(2)}, \mathcal{H}^{(3)}\right\}$ is a $(\boldsymbol{\delta}, \boldsymbol{d}, r)$ regular $(k, 3)$-complex, where $\mathcal{H}^{(1)}=V_{1} \cup V_{2} \cup \ldots \cup V_{k},\left|V_{1}\right|=\left|V_{2}\right|=\ldots=$ $\left|V_{k}\right|=n>n_{0}, \boldsymbol{d}=\left(d_{2}, d_{3}\right)$ and $\boldsymbol{\delta}=\left(\delta_{2}, \delta_{3}\right)$, then $\mathcal{H}^{(3)}$ contains $(1 \pm$ v) $d_{3}^{\left(\begin{array}{c}k \\ 3\end{array}\right)} d_{2}^{\left(\begin{array}{c}k \\ 2\end{array}\right)} n^{k}$ copies of $K_{k}^{(3)}$.

1.4. The main result. Our goal is to prove the following analogy of Theorem 1.13 for 4-cylinders.

Theorem 1.15 (Main Theorem). For any $\nu>0$ the following statement holds.

For every $d_{4} \in(0,1]$, there is a real number $\delta_{4}$ such that for any $d_{3} \in(0,1]$, there exists a real number $\delta_{3}$ such that for any $d_{2} \in(0,1]$, there are $\delta_{2}$ and $n_{0}, r \in \mathbb{N}$ with the property that whenever $\mathcal{H}=\left\{\mathcal{H}^{(1)}, \mathcal{H}^{(2)}, \mathcal{H}^{(3)}, \mathcal{H}^{(4)}\right\}$ is $a(\boldsymbol{\delta}, \boldsymbol{d}, r)$-regular $(5,4)$-complex with vertex set $\mathcal{H}^{(1)}=V_{1} \cup V_{2} \cup V_{3} \cup V_{4} \cup V_{5}$, where $\left|V_{1}\right|=\left|V_{2}\right|=\left|V_{3}\right|=\left|V_{4}\right|=\left|V_{5}\right|=n>n_{0}, \boldsymbol{d}=\left(d_{2}, d_{3}, d_{4}\right)$ and $\boldsymbol{\delta}=\left(\delta_{2}, \delta_{3}, \delta_{4}\right)$, then $\mathcal{H}^{(4)}$ contains

$$
(1 \pm \nu) d_{4}^{\left(\begin{array}{l}
5 \\
4
\end{array}\right)} d_{3}^{\left(\begin{array}{c}
5 \\
3
\end{array}\right)} d_{2}^{\left(\begin{array}{c}
5 \\
2
\end{array}\right)} n^{5}
$$

copies of the complete 4-uniform hypergraph on 5 vertices $K_{5}^{(4)}$.

This theorem has the following intuitive meaning: consider a random (5, 4)-complex $\mathcal{H}=\left\{\mathcal{H}^{(1)}, \mathcal{H}^{(2)}, \mathcal{H}^{(3)}, \mathcal{H}^{(4)}\right\}$ consisting of

i) vertex set $\mathcal{H}^{(1)}=V_{1} \cup V_{2} \cup V_{3} \cup V_{4} \cup V_{5},\left|V_{1}\right|=\left|V_{2}\right|=\left|V_{3}\right|=\left|V_{4}\right|=$ $\left|V_{5}\right|=n$

ii) a random 5-partite graph $\mathcal{H}^{(2)}$, the edges of which are generated with probability $d_{2}$,

iii) a random 5-partite 3 -uniform hypergraph $\mathcal{H}^{(3)}$, whose edges are chosen from triangles of $\mathcal{H}^{(2)}$ independently with probability $d_{3}$, and

iv) a random 5-partite 4-uniform hypergraph $\mathcal{H}^{(4)}$, whose edges are chosen from copies of $K_{4}^{(3)}$ in $\mathcal{H}^{(3)}$ independently with probability $d_{4}$.

It is easy to show that under the above setup, the number of copies of $K_{5}^{(4)}$ in $\mathcal{H}^{(4)}$ is

$$
(1+o(1)) d_{4}^{\left(\begin{array}{l}
5 \\
4
\end{array}\right)} d_{3}^{\left(\begin{array}{c}
5 \\
3
\end{array}\right)} d_{2}^{\left(\begin{array}{c}
5 \\
2
\end{array}\right)} n^{5}
$$

where $o(1) \rightarrow o$ as $n \rightarrow \infty$.

The aim of this paper is to show that the quasi-random properties ensured by the hypergraph regularity lemma [RS04] imply that the number of copies of $K_{5}^{(4)}$ in such a quasi-random $(5,4)$-complex is also given by (1.1).

At the first sight, it seems perhaps surprising that proving (1.1) in a quasirandom setup is significantly more complicated than in a random setup. This difficulty is, however, less surprising if one realizes that Theorem 1.15 quite easily implies a special but already difficult case of Szemerédi's Density Theorem (cf. [Sze75]), namely that for any $\varepsilon>0$ there exists $n_{0}$ such that every $\varepsilon n$ element subset of $\{1, \ldots, n\}, n>n_{0}$, contains an arithmetic progression of length 5 . 
We also remark that all auxiliary results of this paper are easy to prove and understand in the random setup given by i)-iv). Unfortunately, their verification in the quasi-random setup is far from being obvious. This leads us to believe that the following conjecture is true (but it is likely hard to prove).

Conjecture 1.16. For any $\nu>0$ and any $k \in \mathbb{N}$, the following is true:

$\forall d_{k} \in(0,1] \exists \delta_{k} \forall d_{k-1} \in(0,1] \exists \delta_{k-1} \ldots \forall d_{2} \in(0,1] \exists \delta_{2} \exists r \in \mathbb{N} \exists n_{0} \in \mathbb{N}$ such that if $\mathcal{H}=\left\{\mathcal{H}^{(i)}\right\}_{i=1}^{k}$ is a $(\boldsymbol{\delta}, \boldsymbol{d}, r)$-regular $(k+1, k)$-complex with vertex set $\mathcal{H}^{(1)}=V_{1} \cup V_{2} \cup \ldots \cup V_{k+1}$, where $\left|V_{1}\right|=\left|V_{2}\right|=\ldots=\left|V_{k+1}\right|=n>n_{0}$, $\boldsymbol{d}=\left(d_{2}, \ldots, d_{k}\right)$ and $\boldsymbol{\delta}=\left(\delta_{2}, \ldots, \delta_{k}\right)$, then $\mathcal{H}^{(k)}$ contains

$$
(1 \pm \nu) \prod_{s=2}^{k} d_{s}^{\left(\begin{array}{c}
k+1 \\
s
\end{array}\right)} \times n^{k+1}
$$

copies of $K_{k+1}^{(k)}$.

1.5. Extensions of Theorem 1.15. In Definition 1.11, we assumed that for every $I \in[s]^{k}$, the restriction $\mathcal{H}\left[\bigcup_{j \in I} V_{j}\right]$ is $(\delta, d, r)$-regular with respect to $\mathcal{G}\left[\bigcup_{j \in I} V_{j}\right]$. In other words, the density $d_{I}$ of the subgraph $\mathcal{H}\left[\bigcup_{j \in I} V_{j}\right]$ is roughly the same for every $I \in[s]^{k}$. Now we allow different values of $d_{I}$ $\left(I \in[s]^{k}\right)$ and state a straightforward extension of Theorem 1.15. We start with some definitions.

Definition 1.17. Let $\mathcal{G}$ be an $(s, k-1)$-cylinder underlying an $(s, k)$-cylinder $\mathcal{H}, r \in \mathbb{N}$, and let $\vec{d}=\left(d_{I}\right)_{I \in[s]^{k}}$ be a list of $\left(\begin{array}{l}s \\ k\end{array}\right)$ positive real numbers $d_{I}$, where $0<d_{I} \leq 1$. We say that $\mathcal{H}$ is $(\delta, \vec{d}, r)$-regular with respect to $\mathcal{G}$ if $\mathcal{H}\left[\bigcup_{j \in I} V_{j}\right]$ is $\left(\delta, d_{I}, r\right)$-regular with respect to $\mathcal{G}\left[\bigcup_{j \in I} V_{j}\right]$ for all $I \in[s]^{k}$.

Definition 1.18. Let $r \in \mathbb{N}$ and, for every integer $i, 2 \leq i \leq k$, let $\vec{d}_{i}=$ $\left(d_{I}\right)_{I \in[s]^{i}}$ be a list of $\left(\begin{array}{l}s \\ i\end{array}\right)$ positive real numbers $d_{I}$, where $0<d_{I} \leq 1$, and put $\overrightarrow{\boldsymbol{d}}=\left(\vec{d}_{2}, \ldots, \vec{d}_{k}\right)$. Let $\boldsymbol{\delta}=\left(\delta_{2}, \ldots, \delta_{k}\right)$ be a vector of positive real numbers such that $0<\delta_{i}<d_{I}$ for all $I \in[s]^{i}$ and $i=2, \ldots, k$. We say that the $(s, k)$-complex $\mathcal{H}$ is $(\boldsymbol{\delta}, \overrightarrow{\boldsymbol{d}}, r)$-regular if $\mathcal{H}^{(i+1)}$ is $\left(\delta_{i+1}, \vec{d}_{i+1}, r\right)$-regular with respect to $\mathcal{H}^{(i)}$ for every $1 \leq i<k$.

Now we are ready to state a slight extension of Theorem 1.15 .

Theorem 1.19. For any $\nu>0$ the following statement holds.

For every $\vec{d}_{4}=\left(d_{I}\right)_{I \in[5]^{4}} \in(0,1]^{5}$, there is a real number $\delta_{4}$ such that for any $\vec{d}_{3}=\left(d_{I}\right)_{I \in[5]^{3}} \in(0,1]^{10}$, there exists a real number $\delta_{3}$ such that for any $\overrightarrow{d_{2}}=\left(d_{I}\right)_{I \in[5]^{2}} \in(0,1]^{10}$, there are $\delta_{2}$ and $r, n_{0} \in \mathbb{N}$ with the property that whenever $\mathcal{H}=\left\{\mathcal{H}^{(1)}, \mathcal{H}^{(2)}, \mathcal{H}^{(3)}, \mathcal{H}^{(4)}\right\}$ is a $(\boldsymbol{\delta}, \overrightarrow{\boldsymbol{d}}, r)$-regular $(5,4)$-complex with vertex set $\mathcal{H}^{(1)}=V_{1} \cup V_{2} \cup V_{3} \cup V_{4} \cup V_{5}$, where $\left|V_{1}\right|=\left|V_{2}\right|=\left|V_{3}\right|=$ 


$$
\begin{aligned}
\left|V_{4}\right|=\left|V_{5}\right|=n>n_{0}, \overrightarrow{\boldsymbol{d}}= & \left(\overrightarrow{d_{2}}, \overrightarrow{d_{3}}, \vec{d}_{4}\right) \text { and } \boldsymbol{\delta}=\left(\delta_{2}, \delta_{3}, \delta_{4}\right), \text { then } \mathcal{H}^{(4)} \text { contains } \\
& (1 \pm \nu) \prod_{i=2}^{4} \prod_{I \in[5]^{i}} d_{I} \times n^{5}
\end{aligned}
$$

copies of $K_{5}^{(4)}$.

The proof of this theorem follows the lines of the proof of Theorem 1.15 and we omit it here.

For us, the most interesting case occurs when all underlying cylinders have their densities $d_{I}$ independent on the choice of $I$, i.e. $d_{I}=d_{i}$ for every $I \in[5]^{i}$ and $i=2,3$. In this case, the number of $K_{5}^{(4)}$ 's in $\mathcal{H}^{(4)}$ is

$$
(1 \pm \nu) \prod_{I \in[5]^{4}} d_{I} \times d_{3}^{\left(\begin{array}{c}
5 \\
3
\end{array}\right)} d_{2}^{\left(\begin{array}{c}
5 \\
2
\end{array}\right)} n^{5} .
$$

Suppose now that also the densities $d_{I},|I|=4$, are independent on the choice of $I$, i.e. $d_{I}=d_{4}$ for every $I \in[5]^{4}$.

Let $\mathcal{G}$ be an arbitrary 4 -uniform hypergraph on 5 vertices $v_{1}, \ldots, v_{5}$. We define a $(5,4)$-cylinder $\widetilde{\mathcal{H}}^{(4)}=\widetilde{\mathcal{H}}^{(4)}(\mathcal{G})$ in the following way. For every $I \in[5]^{4}$, we set

$$
\widetilde{\mathcal{H}}^{(4)}\left[\bigcup_{i \in I} V_{i}\right]= \begin{cases}\mathcal{H}\left[\bigcup_{i \in I} V_{i}\right] & \text { if }\left\{v_{i}: i \in I\right\} \in E(\mathcal{G}), \\ \mathcal{K}_{4}\left(\mathcal{H}^{(3)}\left[\bigcup_{i \in I} V_{i}\right]\right) \backslash \mathcal{H}\left[\bigcup_{i \in I} V_{i}\right] & \text { otherwise. }\end{cases}
$$

Observe that the density of $\widetilde{\mathcal{H}}^{(4)}\left[\bigcup_{i \in I} V_{i}\right]$ is $d_{4}$ if $\left\{v_{i}: i \in I\right\} \in E(\mathcal{G})$, and it is $1-d_{4}$ otherwise. Applying (1.2), we get

$$
\left|\mathcal{K}_{5}\left(\widetilde{\mathcal{H}}^{(4)}(\mathcal{G})\right)\right|=(1 \pm \nu) d_{4}^{e(\mathcal{G})}\left(1-d_{4}\right)^{\left(\begin{array}{l}
5 \\
4
\end{array}\right)-e(\mathcal{G})} d_{3}^{\left(\begin{array}{c}
5 \\
3
\end{array}\right)} d_{2}^{\left(\begin{array}{c}
5 \\
2
\end{array}\right)} n^{5} .
$$

Consequently, we deduce the following counting formula.

Corollary 1.20 (Subhypergraph counting formula). For any $\nu>0$ and any 4-uniform hypergraph $\mathcal{G}$ on 5 vertices with automorphism group $\operatorname{Aut}(\mathcal{G})$, the following statement holds.

For every $d_{4} \in(0,1]$, there is a real number $\delta_{4}$ such that for any $d_{3} \in(0,1]$, there exists a real number $\delta_{3}$ such that for any $d_{2} \in(0,1]$, there are $\delta_{2}$ and $r, n_{0} \in \mathbb{N}$ with the property that whenever $\mathcal{H}=\left\{\mathcal{H}^{(1)}, \mathcal{H}^{(2)}, \mathcal{H}^{(3)}, \mathcal{H}^{(4)}\right\}$ is a $(\boldsymbol{\delta}, \boldsymbol{d}, r)$-regular $(5,4)$-complex with vertex set $\mathcal{H}^{(1)}=V_{1} \cup V_{2} \cup V_{3} \cup V_{4} \cup V_{5}$, where $\left|V_{1}\right|=\left|V_{2}\right|=\left|V_{3}\right|=\left|V_{4}\right|=\left|V_{5}\right|=n>n_{0}, \boldsymbol{d}=\left(d_{2}, d_{3}, d_{4}\right)$ and $\boldsymbol{\delta}=\left(\delta_{2}, \delta_{3}, \delta_{4}\right)$, then $\mathcal{H}^{(4)}$ contains

$$
(1 \pm \nu) d_{4}^{e(\mathcal{G})}\left(1-d_{4}\right)^{\left(\begin{array}{l}
5 \\
4
\end{array}\right)-e(\mathcal{G})} d_{3}^{\left(\begin{array}{l}
5 \\
3
\end{array}\right)} d_{2}^{\left(\begin{array}{l}
5 \\
2
\end{array}\right)} n^{5} \frac{5 !}{|\operatorname{Aut}(\mathcal{G})|}
$$

induced copies of $\mathcal{G}$ whose vertex sets are crossing.

Similarly, if we set $d_{I}=d_{2}$ for all $I \in[5]^{2}, d_{I}=d_{3}$ for all $I \in[5]^{3}$, and we know that $d_{I} \geq d_{4}$ for all $I \in[5]^{4}$ in Theorem 1.19, then we obtain the following corollary. 
Corollary 1.21. For any $\nu>0$ the following statement holds.

For every $d_{4} \in(0,1]$ there exists $\delta_{4}$ such that for every $d_{3} \in(0,1]$ there exists $\delta_{3}$ such that for every $d_{2} \in(0,1]$ there exist $\delta_{2}$ and $r, n_{0} \in \mathbb{N}$ so that whenever $\mathcal{H}=\left\{\mathcal{H}^{(1)}, \mathcal{H}^{(2)}, \mathcal{H}^{(3)}, \mathcal{H}^{(4)}\right\}$ is a $(\boldsymbol{\delta}, \overrightarrow{\boldsymbol{d}}, r)$-regular $(5,4)$-complex, where

- $\mathcal{H}^{(1)}=V_{1} \cup V_{2} \cup V_{3} \cup V_{4} \cup V_{5}$ and $\left|V_{1}\right|=\left|V_{2}\right|=\left|V_{3}\right|=\left|V_{4}\right|=\left|V_{5}\right|=$ $n>n_{0}$

- $\vec{d}_{4}=\left(d_{I}\right)_{I \in[5]^{4}}$ and $d_{I} \geq d_{4}$ for every $I \in[5]^{4}$,

- $\overrightarrow{d_{3}}=\left(d_{I}\right)_{I \in[5]^{3}}$ and $d_{I}=d_{3}$ for every $I \in[5]^{3}$,

- $\overrightarrow{d_{2}}=\left(d_{I}\right)_{I \in[5]^{2}}$ and $d_{I}=d_{2}$ for every $I \in[5]^{2}$,

- $\overrightarrow{\boldsymbol{d}}=\left(\vec{d}_{2}, \vec{d}_{3}, \vec{d}_{4}\right)$ and $\boldsymbol{\delta}=\left(\delta_{2}, \delta_{3}, \delta_{4}\right)$,

then $\mathcal{H}^{(4)}$ contains at least

$$
(1-\nu) d_{4}^{\left(\begin{array}{l}
5 \\
4
\end{array}\right)} d_{3}^{\left(\begin{array}{c}
5 \\
3
\end{array}\right)} d_{2}^{\left(\begin{array}{c}
5 \\
2
\end{array}\right)} n^{5}
$$

copies of $K_{5}^{(4)}$.

\section{Auxiliary Results}

The goal of this section is to present our tools for the proof of the Main Theorem. We first state all necessary concepts and then propositions that we later use in the actual proof. One of the central concepts in the proof of Theorem 1.15 is the notion of the link of a vertex.

Definition 2.1. Let $\mathcal{G}$ be a $k$-uniform hypergraph and $x \in V(\mathcal{G})$. We will call the set

$$
\mathcal{G}(x)=\{e \backslash\{x\}: e \in \mathcal{G}, x \in e\}
$$

the link of the vertex $x$ in $\mathcal{G}$. Note that $\mathcal{G}(x)$ is a $(k-1)$-uniform hypergraph. Moreover, if $\mathcal{G}$ is an $(s, k)$-cylinder, then $\mathcal{G}(x)$ is an $(s-1, k-1)$-cylinder. For a subset $W \subset V(\mathcal{G})$, we define $\mathcal{G}(W)$ by

$$
\mathcal{G}(W)=\bigcap_{x \in W} \mathcal{G}(x)
$$

For simplicity, if $W=\left\{x_{1}, \ldots, x_{k}\right\}$, we write $\mathcal{G}\left(x_{1}, \ldots, x_{k}\right)$.

Through the remainder of the paper we fix a $(\boldsymbol{\delta}, \boldsymbol{d}, r)$-regular $(5,4)$-complex $\mathcal{H}=\left\{\mathcal{H}^{(1)}, \mathcal{H}^{(2)}, \mathcal{H}^{(3)}, \mathcal{H}^{(4)}\right\}$ and $\nu>0$, and we will assume that $\mathcal{H}^{(1)}=$ $V_{1} \cup \ldots \cup V_{5}$ and $\left|V_{1}\right|=\ldots=\left|V_{5}\right|=n>n_{0}$. The purpose of this condition is to simplify the proof and all statements remain valid for partite sets with different sizes.

Let us recall the quantification of the constants in Theorem 1.15:

$$
\forall d_{4} \exists \delta_{4} \forall d_{3} \exists \delta_{3} \forall d_{2} \exists \delta_{2} \exists r \exists n_{0} .
$$


Due to this quantification we may assume

$$
\begin{gathered}
\nu \gg \delta_{4}>0, \\
1>d_{4} \gg \delta_{4}>0,1>d_{3} \gg \delta_{3}>0,1>d_{2} \gg \delta_{2}>0 \\
r \gg \max \left\{1 / d_{4}, 1 / d_{3}, 1 / d_{2}\right\}, \\
n_{0} \text { is sufficiently large. }
\end{gathered}
$$

Since one usually applies Theorem 1.15 after applying the Regularity Lemma (cf. Theorem 7.15 in [RS04]), we may assume the following order of constants that is guaranteed by this Lemma.

$$
d_{4} \gg \delta_{4} \gg d_{3} \gg \delta_{3} \gg d_{2} \gg \delta_{2}>0 .
$$

We remark, however, that this order is not crucial for the proof and the authors decided to use it only for the sake of clearer presentation.

Our proof will be based on the following four propositions. The first proposition states that for almost all vertices $x$, the number of copies of $K_{4}$ induced on the neighborhood $\mathcal{H}^{(2)}(x)$ of $x$ in $\mathcal{H}^{(2)}$ is bounded.

Proposition 2.2. For all but at most $8 \delta_{2}^{1 / 2} n$ vertices $x \in V_{1}$

$$
\left|\mathcal{K}_{4}\left(\mathcal{H}^{(2)}\left[\mathcal{H}^{(2)}(x)\right]\right)\right| \leq 2 d_{2}^{\left(\begin{array}{c}
5 \\
2
\end{array}\right)} n^{4}
$$

Next proposition bounds the number of copies of $K_{4}$ induced on the joint neighborhood of a pair $x, x^{\prime}$ in $\mathcal{H}^{(2)}$ for almost all pairs of vertices $x, x^{\prime}$.

Proposition 2.3. For all but at most $16 \delta_{2}^{1 / 2} n^{2}$ pairs of vertices $x, x^{\prime} \in V_{1}$

$$
\left|\mathcal{K}_{4}\left(\mathcal{H}^{(2)}\left[\mathcal{H}^{(2)}\left(x, x^{\prime}\right)\right]\right)\right| \leq 2 d_{2}^{14} n^{4} .
$$

The third proposition shows that for almost all pairs $x, x^{\prime}$, the number of copies of $K_{4}^{(3)}$ induced by those 3-edges of $\mathcal{H}^{(3)}$ which are also triangles in the link $\mathcal{H}^{(3)}\left(x, x^{\prime}\right)$, is bounded.

Proposition 2.4. For all but at most $60 \delta_{3}^{1 / 16} n^{2}$ pairs of vertices $x, x^{\prime} \in V_{1}$

$$
\left|\mathcal{K}_{4}\left(\mathcal{H}^{(3)} \cap \mathcal{K}_{3}\left(\mathcal{H}^{(3)}\left(x, x^{\prime}\right)\right)\right)\right| \leq 2 d_{3}^{16} d_{2}^{14} n^{4}
$$

The last proposition shows that the number of copies of $K_{4}^{(3)}$ in the link $\mathcal{H}^{(4)}(x)$ is roughly the same for almost all vertices $x$.

Proposition 2.5. For all but but at most $100 \delta_{4}^{1 / 4} n$ vertices $x \in V_{1}$

$$
\left|\mathcal{K}_{4}\left(\mathcal{H}^{(4)}(x)\right)\right|=(1 \pm \nu / 2) d_{4}^{4} d_{3}^{\left(\begin{array}{l}
5 \\
3
\end{array}\right)} d_{2}^{\left(\begin{array}{c}
5 \\
2
\end{array}\right)} n^{4}
$$

Since the proofs of these propositions are rather complex, we defer them until later. Propositions 2.2 and 2.3 are proved in Section 4. In order to prove Propositions 2.5 and 2.4, we need the so called $\ell$-graphs Lemma (see Section 5) and a number of additional claims about $(s, 3)$-cylinders (see Sections 6, 7 and 9) and $(s, 4)$-cylinders (see Section 10). Therefore, the 
proof of Propositions 2.4 is in Section 8, and the proof of Proposition 2.5 is given in Section 11.

We will also need the following lemma which follows from Markov's inequality.

Lemma 2.6 (Picking Lemma, [PRS04]). Let $V$ be a set of size $m, k$ be a nonnegative integer, and $\mathcal{P}_{1}, \ldots \mathcal{P}_{k}$ be arbitrary graphs on $V$. Furthermore, suppose that $\left|\mathcal{P}_{1}\right| \leq \sigma_{1} m^{2},\left|\mathcal{P}_{2}\right| \leq \sigma_{2} m^{2}, \ldots,\left|\mathcal{P}_{k}\right| \leq \sigma_{k} m^{2}$. Then for every subset $W \subset V$ with at least cm elements and a positive integer $t$ such that

$$
\frac{2 \sigma_{1} t^{2}}{c^{2}}<\frac{1}{k}
$$

there exists a choice of $t$ vertices $x_{1}, x_{2}, \ldots, x_{t} \in W$ such that

(i) $\left\{x_{u}, x_{v}\right\} \notin \mathcal{P}_{1}$ for all $1 \leq u<v \leq t$,

(ii) for all $i \in[k] \backslash\{1\},\left\{x_{u}, x_{v}\right\} \notin \mathcal{P}_{i}$ for all but at most $\left(2 k \sigma_{i} / c^{2}\right) t^{2}$ pairs $1 \leq u<v \leq t$.

In our proofs, we will also need the following observation, which is an easy consequence of the Inclusion-Exclusion Principle.

Observation 2.7. Let $X$ be a set and $A_{1}, \ldots, A_{t} t$ of its arbitrary finite subsets. Then

$$
\left|\bigcup_{i=1}^{t} A_{i}\right| \geq \sum_{i=1}^{t}\left|A_{i}\right|-\sum_{1 \leq i<j \leq t}\left|A_{i} \cap A_{j}\right| .
$$

Furthermore, if $a \times \sum_{i=1}^{t}\left|A_{i}\right|-\sum_{1 \leq i<j \leq t}\left|A_{i} \cap A_{j}\right| \geq 0$ for some $a \in(0,1)$, then

$$
\left|\bigcup_{i=1}^{t} A_{i}\right| \geq(1-a) \sum_{i=1}^{t}\left|A_{i}\right| .
$$

Now we are ready to prove the Main Theorem.

\section{Proof of the Main Theorem}

3.1. Lower bound. Let $W$ be the set of all vertices $x \in V_{1}$ satisfying inequality (2.7). Thus, for every vertex $x \in W$ we have:

$$
\left|\mathcal{K}_{4}\left(\mathcal{H}^{(4)}(x)\right)\right|=(1 \pm \nu / 2) d_{4}^{4} d_{3}^{\left(\begin{array}{c}
5 \\
3
\end{array}\right)} d_{2}^{\left(\begin{array}{c}
5 \\
2
\end{array}\right)} n^{4} .
$$

By Proposition 2.5 we know that

$$
|W| \geq\left(1-100 \delta_{4}^{1 / 4}\right) n .
$$

Since the proof is rather complex and long, we outline its idea first. For every vertex $x \in W$ there are $(1 \pm \nu / 2) d_{4}^{4} d_{3}^{\left(\begin{array}{c}5 \\ 3\end{array}\right)} d_{2}^{\left(\begin{array}{c}5 \\ 2\end{array}\right)} n^{4}$ copies of $K_{4}^{(3)}$ in $\mathcal{H}^{(4)}(x)$. Notice that every such $K_{4}^{(3)}$ together with $x$ form a copy of $\left(K_{5}^{(4)} \backslash\right.$ edge $)$ in $\mathcal{H}^{(4)}$. Therefore, we would like to apply the $\left(\delta_{4}, d_{4}, r\right)$-regularity of $\mathcal{H}^{(4)}$ on these copies to obtain the uncounted for edge. 
The number of copies of $K_{4}^{(3)}$ in $\mathcal{H}^{(4)}(x)$ is, however, insufficient to apply the $\left(\delta_{4}, d_{4}, r\right)$-regularity of $\mathcal{H}^{(4)}$. Indeed, from Theorem 1.13 we have $\left|\mathcal{K}_{4}\left(\partial_{1} \mathcal{H}^{(3)}\right)\right| \geq(1 / 2) d_{2}^{6} d_{3}^{4} n^{4}$. To apply the $\left(\delta_{4}, d_{4}, r\right)$-regularity of $\mathcal{H}^{(4)}$, we need to satisfy

$$
\left|\mathcal{K}_{4}\left(\mathcal{H}^{(4)}(x)\right)\right| \geq \delta_{4}\left|\mathcal{K}_{4}\left(\partial_{1} \mathcal{H}^{(3)}\right)\right| .
$$

Since $\left|\mathcal{K}_{4}\left(\mathcal{H}^{(4)}(x)\right)\right| \leq(1+\nu / 2) d_{4}^{4} d_{3}^{\left(\begin{array}{c}5 \\ 3\end{array}\right)} d_{2}^{\left(\begin{array}{c}5 \\ 2\end{array}\right)} n^{4}$, we obtain

$$
(1+\nu / 2) d_{4}^{4} d_{3}^{\left(\begin{array}{c}
5 \\
3
\end{array}\right)} d_{2}^{\left(\begin{array}{c}
5 \\
2
\end{array}\right)} n^{4} \geq \delta_{4} \times(1 / 2) d_{2}^{6} d_{3}^{4} n^{4}
$$

or $(1+\nu / 2) d_{4}^{4} d_{3}^{6} d_{2}^{4} \geq(1 / 2) \delta_{4}$. This is impossible to satisfy due to the order of constants and quantification of this theorem.

Thus, we must use the full power of $r$-regularity. We select $r=2 \delta_{4}^{1 / 2} /\left(d_{2}^{4} d_{3}^{6}\right)$ vertices $x_{1}, \ldots, x_{r}$ from $W$ in such a way that the size of $\bigcup_{j=1}^{r} \mathcal{K}_{4}\left(\mathcal{H}^{(4)}\left(x_{j}\right)\right)$ is sufficiently large to apply the regularity of $\mathcal{H}^{(4)}$, i.e.

$$
\left|\bigcup_{j=1}^{r} \mathcal{K}_{4}\left(\mathcal{H}^{(4)}\left(x_{j}\right)\right)\right| \geq \delta_{4}\left|\mathcal{K}_{4}\left(\partial_{1} \mathcal{H}^{(3)}\right)\right| .
$$

In order to choose this $r$-tuple of vertices with a large union, we will use the Picking Lemma and the fact that

$\left|\bigcup_{j=1}^{r} \mathcal{K}_{4}\left(\mathcal{H}^{(4)}\left(x_{j}\right)\right)\right| \geq \sum_{j=1}^{r}\left|\mathcal{K}_{4}\left(\mathcal{H}^{(4)}\left(x_{j}\right)\right)\right|-\sum_{1 \leq i<j \leq r}\left|\mathcal{K}_{4}\left(\mathcal{H}^{(4)}\left(x_{i}\right)\right) \cap \mathcal{K}_{4}\left(\mathcal{H}^{(4)}\left(x_{j}\right)\right)\right|$.

The Picking Lemma and Proposition 2.5 will guarantee the choice of the $r$-tuple $x_{1}, \ldots, x_{r}$ for which $\sum_{j=1}^{r}\left|\mathcal{K}_{4}\left(\mathcal{H}^{(4)}\left(x_{j}\right)\right)\right|$ is "large", whereas the same lemma and Propositions 2.3 and 2.4 will make the second term $\sum_{1 \leq i<j \leq r}\left|\mathcal{K}_{4}\left(\mathcal{H}^{(4)}\left(x_{i}\right)\right) \cap \mathcal{K}_{4}\left(\mathcal{H}^{(4)}\left(x_{j}\right)\right)\right|$ "small".

Since (3.2) holds, we will be able to apply the $\left(\delta_{4}, d_{4}, r\right)$-regularity of $\mathcal{H}^{(4)}$ to obtain

$$
\left|\mathcal{H}^{(4)} \cap \bigcup_{j=1}^{r} \mathcal{K}_{4}\left(\mathcal{H}^{(4)}\left(x_{j}\right)\right)\right|=\left(d_{4} \pm \delta_{4}\right)\left|\bigcup_{j=1}^{r} \mathcal{K}_{4}\left(\mathcal{H}^{(4)}\left(x_{j}\right)\right)\right| .
$$

Observe that the left-hand side of the above equation counts the number of copies of $K_{5}^{(4)}$ that use one of $x_{1}, \ldots, x_{r}$ as a vertex. Also note that this number is $O\left(n^{4}\right)$ which is far less than what Theorem 1.15 promises. To get a full amount of copies of $K_{5}^{(4)}$ as claimed by the Theorem, we will iterate this process as long as we are able to use the Picking Lemma.

After describing the idea, we start with a detailed proof. We define two graphs $\mathcal{P}_{1}$ and $\mathcal{P}_{2}$, both with vertex set $V_{1}$ and edge sets defined by:

$$
\begin{aligned}
& E\left(\mathcal{P}_{1}\right)=\left\{x x^{\prime}:\left|\mathcal{K}_{4}\left(\mathcal{H}^{(2)}\left[\mathcal{H}^{(2)}\left(x, x^{\prime}\right)\right]\right)\right|>2 d_{2}^{14} n^{4}\right\} \\
& E\left(\mathcal{P}_{2}\right)=\left\{x x^{\prime}:\left|\mathcal{K}_{4}\left(\mathcal{H}^{(3)} \cap \mathcal{K}_{3}\left(\mathcal{H}^{(3)}\left(x, x^{\prime}\right)\right)\right)\right|>2 d_{3}^{16} d_{2}^{14} n^{4}\right\} .
\end{aligned}
$$


It follows from Propositions 2.3 and 2.4 that the sizes of $\mathcal{P}_{1}$ and $\mathcal{P}_{2}$ are bounded, more precisely, $\left|\mathcal{P}_{1}\right| \leq 16 \delta_{2}^{1 / 2} n^{2}$ and $\left|\mathcal{P}_{2}\right| \leq 60 \delta_{3}^{1 / 16} n^{2}$.

We apply the Picking Lemma on $W=V_{1}$ with parameters $\sigma_{1}=16 \delta_{2}^{1 / 2}$, $\sigma_{2}=60 \delta_{3}^{1 / 16}, t=r=2 \delta_{4}^{1 / 2} /\left(d_{2}^{4} d_{3}^{6}\right), c=\delta_{4}^{1 / 2}$, and obtain $r$ vertices $x_{1}, \ldots, x_{r} \in W$ such that all pairs $\left\{x_{i}, x_{j}\right\}$ satisfy

$$
\left|\mathcal{K}_{4}\left(\mathcal{H}^{(2)}\left[\mathcal{H}^{(2)}\left(x_{i}, x_{j}\right)\right]\right)\right| \leq 2 d_{2}^{14} n^{4},
$$

and all but $\left(2 \times 2 \times 60 \delta_{3}^{1 / 16} / \delta_{4}\right) r^{2} \leq \delta_{3}^{1 / 32} r^{2}$ pairs $\left\{x_{i}, x_{j}\right\}$ satisfy

$$
\left|\mathcal{K}_{4}\left(\mathcal{H}^{(3)} \cap \mathcal{K}_{3}\left(\mathcal{H}^{(3)}\left(x_{i}, x_{j}\right)\right)\right)\right| \leq 2 d_{3}^{16} d_{2}^{14} n^{4} .
$$

This is possible as long as

$$
|W| \geq c \times n=\delta_{4}^{1 / 2} n .
$$

Now we estimate the size of $\bigcup_{j=1}^{r} \mathcal{K}_{4}\left(\mathcal{H}^{(4)}\left(x_{j}\right)\right)$. We first apply Observation 2.7:

$\left|\bigcup_{j=1}^{r} \mathcal{K}_{4}\left(\mathcal{H}^{(4)}\left(x_{j}\right)\right)\right| \geq \sum_{j=1}^{r}\left|\mathcal{K}_{4}\left(\mathcal{H}^{(4)}\left(x_{j}\right)\right)\right|-\sum_{1 \leq i<j \leq r}\left|\mathcal{K}_{4}\left(\mathcal{H}^{(4)}\left(x_{i}\right)\right) \cap \mathcal{K}_{4}\left(\mathcal{H}^{(4)}\left(x_{j}\right)\right)\right|$.

The next step is to estimate both terms on the right-hand side. The first term is easier to handle, we use inequality (3.1):

$$
\sum_{j=1}^{r}\left|\mathcal{K}_{4}\left(\mathcal{H}^{(4)}\left(x_{j}\right)\right)\right| \geq r \times(1-\nu / 2) d_{4}^{4} d_{3}^{\left(\begin{array}{c}
5 \\
3
\end{array}\right)} d_{2}^{\left(\begin{array}{c}
5 \\
2
\end{array}\right)} n^{4} .
$$

To get an estimate for the second term, we must observe several facts:

- $\mathcal{K}_{4}\left(\mathcal{H}^{(4)}\left(x_{i}\right)\right) \cap \mathcal{K}_{4}\left(\mathcal{H}^{(4)}\left(x_{j}\right)\right)=\mathcal{K}_{4}\left(\mathcal{H}^{(4)}\left(x_{i}, x_{j}\right)\right)$ for every $1 \leq i<$ $j \leq r$.

- $K_{4}^{(3)}$ in $\mathcal{H}^{(4)}\left(x_{i}, x_{j}\right)$ is also a copy of $K_{4}^{(3)}$ in $\mathcal{H}^{(3)} \cap \mathcal{K}_{3}\left(\mathcal{H}^{(3)}\left(x_{i}, x_{j}\right)\right)$.

- $K_{4}^{(3)}$ in $\mathcal{H}^{(4)}\left(x_{i}, x_{j}\right)$ is also a copy of $K_{4}^{(3)}$ in $\mathcal{K}_{3}\left(\mathcal{H}^{(2)}\left[\mathcal{H}^{(2)}\left(x_{i}, x_{j}\right)\right]\right)$.

Since we know that all but at most $\delta_{3}^{1 / 32} r^{2}$ pairs $\left\{x_{i}, x_{j}\right\}$ satisfy (3.4), for these pairs we use the estimate

$$
\left|\mathcal{K}_{4}\left(\mathcal{H}^{(4)}\left(x_{i}, x_{j}\right)\right)\right| \leq\left|\mathcal{K}_{4}\left(\mathcal{H}^{(3)} \cap \mathcal{K}_{3}\left(\mathcal{H}^{(3)}\left(x_{i}, x_{j}\right)\right)\right)\right| \leq 2 d_{3}^{16} d_{2}^{14} n^{4} .
$$

The remaining $\delta_{3}^{1 / 32} r^{2}$ pairs $\left\{x_{i}, x_{j}\right\}$ satisfy inequality (3.3), thus we estimate $\left|\mathcal{K}_{4}\left(\mathcal{H}^{(4)}\left(x_{i}, x_{j}\right)\right)\right|$ as

$$
\left|\mathcal{K}_{4}\left(\mathcal{H}^{(4)}\left(x_{i}, x_{j}\right)\right)\right| \leq\left|\mathcal{K}_{4}\left(\mathcal{H}^{(2)}\left[\mathcal{H}^{(2)}\left(x_{i}, x_{j}\right)\right]\right)\right| \leq 2 d_{2}^{14} n^{4} .
$$

Now we combine (3.7) and (3.8) to obtain

$$
\sum_{1 \leq i<j \leq r}\left|\mathcal{K}_{4}\left(\mathcal{H}^{(4)}\left(x_{i}\right)\right) \cap \mathcal{K}_{4}\left(\mathcal{H}^{(4)}\left(x_{j}\right)\right)\right| \leq\left(\begin{array}{l}
r \\
2
\end{array}\right) \times 2 d_{3}^{16} d_{2}^{14} n^{4}+\delta_{3}^{1 / 32} r^{2} \times 2 d_{2}^{14} n^{4} .
$$


We use the assumption $\delta_{3} \ll d_{3}$ and conclude that $\delta_{3}^{1 / 32} r^{2} \times 2 d_{2}^{14} n^{4} \leq$ $d_{3}^{16} r^{2} d_{2}^{14} n^{4}$. Then,

$$
\sum_{1 \leq i<j \leq r}\left|\mathcal{K}_{4}\left(\mathcal{H}^{(4)}\left(x_{i}\right)\right) \cap \mathcal{K}_{4}\left(\mathcal{H}^{(4)}\left(x_{j}\right)\right)\right| \leq 2 r^{2} d_{3}^{16} d_{2}^{14} n^{4} .
$$

Using (3.6), (3.9), and the definition of $r$ (recall $r=2 \delta_{4}^{1 / 2} /\left(d_{2}^{4} d_{3}^{6}\right)$ ), we obtain that

$$
\begin{aligned}
\left|\bigcup_{j=1}^{r} \mathcal{K}_{4}\left(\mathcal{H}^{(4)}\left(x_{j}\right)\right)\right| & \geq r \times(1-\nu / 2) d_{4}^{4} d_{3}^{\left(\begin{array}{l}
5 \\
3
\end{array}\right)} d_{2}^{\left(\begin{array}{l}
5 \\
2
\end{array}\right)} n^{4}-2 r^{2} d_{3}^{16} d_{2}^{14} n^{4} \\
& \geq \delta_{4}^{1 / 2} d_{4}^{4} d_{3}^{4} d_{2}^{6} n^{4}-8 \delta_{4} d_{3}^{4} d_{2}^{6} n^{4} \stackrel{(2.3 \mathrm{a})}{\geq} 2 \delta_{4} d_{3}^{4} d_{2}^{6} n^{4} .
\end{aligned}
$$

On the other hand, we observe that $(4,3)$-cylinder $\partial_{1} \mathcal{H}^{(3)}$, i.e. the subgraph of $\mathcal{H}^{(3)}$ induced on $\bigcup_{j \neq 1} V_{j}$, is $\left(\delta_{3}, d_{3}, r\right)$-regular with respect to $\partial_{1} \mathcal{H}^{(2)}$, and that $\partial_{1} \mathcal{H}^{(2)}$ is $\left(\delta_{2}, d_{2}\right)$-regular. Furthermore, the quantification of this theorem allows us to choose $\delta_{3}$ and $\delta_{2}$ so that the assumptions of Theorem 1.13 are satisfied. Consequently, we infer that $\left|\mathcal{K}_{4}\left(\partial_{1} \mathcal{H}^{(3)}\right)\right| \leq 2 d_{3}^{4} d_{2}^{6} n^{4}$. Therefore,

$$
\left|\bigcup_{j=1}^{r} \mathcal{K}_{4}\left(\mathcal{H}^{(4)}\left(x_{j}\right)\right)\right| \geq \delta_{4}\left|\mathcal{K}_{4}\left(\partial_{1} \mathcal{H}^{(3)}\right)\right|,
$$

so, by the $\left(\delta_{4}, d_{4}, r\right)$-regularity of $\mathcal{H}^{(4)}$ with respect to $\mathcal{H}^{(3)}$, we obtain

$$
\left|\mathcal{H}^{(4)} \cap \bigcup_{j=1}^{r} \mathcal{K}_{4}\left(\mathcal{H}^{(4)}\left(x_{j}\right)\right)\right|=\left(d_{4} \pm \delta_{4}\right)\left|\bigcup_{j=1}^{r} \mathcal{K}_{4}\left(\mathcal{H}^{(4)}\left(x_{j}\right)\right)\right| \text {. }
$$

From the above inequality and (3.1), one can easily conclude:

$$
\begin{aligned}
\mid \mathcal{H}^{(4)} & \cap \bigcup_{j=1}^{r} \mathcal{K}_{4}\left(\mathcal{H}^{(4)}\left(x_{j}\right)\right)\left|\leq\left(d_{4}+\delta_{4}\right)\right| \bigcup_{j=1}^{r} \mathcal{K}_{4}\left(\mathcal{H}^{(4)}\left(x_{j}\right)\right) \mid \\
& \leq\left(d_{4}+\delta_{4}\right) \sum_{j=1}^{r}\left|\mathcal{K}_{4}\left(\mathcal{H}^{(4)}\left(x_{j}\right)\right)\right| \leq\left(d_{4}+\delta_{4}\right) r(1+\nu / 2) d_{4}^{4} d_{3}^{\left(\begin{array}{c}
5 \\
3
\end{array}\right)} d_{2}^{\left(\begin{array}{c}
5 \\
2
\end{array}\right)} n^{4} .
\end{aligned}
$$

In order to get a lower bound on $\left|\mathcal{H}^{(4)} \cap \bigcup_{j=1}^{r} \mathcal{K}_{4}\left(\mathcal{H}^{(4)}\left(x_{j}\right)\right)\right|$, we first use (3.11):

$$
\left|\mathcal{H}^{(4)} \cap \bigcup_{j=1}^{r} \mathcal{K}_{4}\left(\mathcal{H}^{(4)}\left(x_{j}\right)\right)\right| \geq\left(d_{4}-\delta_{4}\right)\left|\bigcup_{j=1}^{r} \mathcal{K}_{4}\left(\mathcal{H}^{(4)}\left(x_{j}\right)\right)\right| .
$$

Second, we want to apply Observation 2.7 with $a=\delta_{4}^{1 / 4}$ and obtain:

$$
\left|\bigcup_{j=1}^{r} \mathcal{K}_{4}\left(\mathcal{H}^{(4)}\left(x_{j}\right)\right)\right| \geq\left(1-\delta_{4}^{1 / 4}\right) \sum_{j=1}^{r}\left|\mathcal{K}_{4}\left(\mathcal{H}^{(4)}\left(x_{j}\right)\right)\right| .
$$


In order to do this, we must show that

$$
\delta_{4}^{1 / 4} \times \sum_{j=1}^{r}\left|\mathcal{K}_{4}\left(\mathcal{H}^{(4)}\left(x_{j}\right)\right)\right|-\sum_{1 \leq i<j \leq r}\left|\mathcal{K}_{4}\left(\mathcal{H}^{(4)}\left(x_{i}\right)\right) \cap \mathcal{K}_{4}\left(\mathcal{H}^{(4)}\left(x_{j}\right)\right)\right| \geq 0 .
$$

This is easy to verify using (3.6), (3.9), and $d_{4} \gg \delta_{4}$. Indeed,

$$
\delta_{4}^{1 / 4} \times \sum_{j=1}^{r}\left|\mathcal{K}_{4}\left(\mathcal{H}^{(4)}\left(x_{j}\right)\right)\right| \stackrel{(3.6)}{\geq} \delta_{4}^{1 / 4} \times \delta_{4}^{1 / 2} d_{4}^{4} d_{3}^{4} d_{2}^{6} n^{4} \geq 8 \delta_{4} d_{3}^{4} d_{2}^{6} n^{4}
$$

and

$$
\sum_{1 \leq i<j \leq r}\left|\mathcal{K}_{4}\left(\mathcal{H}^{(4)}\left(x_{i}\right)\right) \cap \mathcal{K}_{4}\left(\mathcal{H}^{(4)}\left(x_{j}\right)\right)\right| \stackrel{(3.9)}{\leq} 2 r^{2} d_{3}^{16} d_{2}^{14} n^{4} \leq 8 \delta_{4} d_{3}^{4} d_{2}^{6} n^{4} .
$$

Then, we combine inequalities (3.12) and (3.13) and get

$$
\left|\mathcal{H}^{(4)} \cap \bigcup_{j=1}^{r} \mathcal{K}_{4}\left(\mathcal{H}^{(4)}\left(x_{j}\right)\right)\right| \geq d_{4}\left(1-\delta_{4}^{1 / 4}\right)^{2} \sum_{j=1}^{r}\left|\mathcal{K}_{4}\left(\mathcal{H}^{(4)}\left(x_{j}\right)\right)\right| .
$$

Combining the last inequality with (3.6) yields

$$
\left|\mathcal{H}^{(4)} \cap \bigcup_{j=1}^{r} \mathcal{K}_{4}\left(\mathcal{H}^{(4)}\left(x_{j}\right)\right)\right| \stackrel{(3.6)}{\geq}\left(1-\delta_{4}^{1 / 4}\right)^{2} \times r \times(1-\nu / 2) d_{4}^{5} d_{3}^{\left(\begin{array}{l}
5 \\
3
\end{array}\right)} d_{2}^{\left(\begin{array}{l}
5 \\
2
\end{array}\right)} n^{4} .
$$

So far we produced only const. $\times n^{4}$ cliques $K_{5}^{(4)}$, while we promised to deliver const. $\times n^{5}$. Therefore, we remove vertices $x_{1}, \ldots, x_{r}$ from $W$ and iterate the whole process again. Due to (3.5), we can repeat this process as long as $|W|>\delta_{4}^{1 / 2} n$. This way we produce a sequence of at least (1$\left.100 \delta_{4}^{1 / 4}-\delta_{4}^{1 / 2}\right) n / r$ but not more than $n / r$-tuples $X^{(1)}=\left\{x_{1}, \ldots, x_{r}\right\}=$ $\left\{x_{1}^{(1)}, \ldots, x_{r}^{(1)}\right\}, X^{(2)}=\left\{x_{1}^{(2)}, \ldots, x_{r}^{(2)}\right\}$, etc. Analogously to (3.14), each iteration produces at least

$$
\left(1-\delta_{4}^{1 / 4}\right)^{2} \times r \times(1-\nu / 2) d_{4}^{5} d_{3}^{\left(\begin{array}{l}
5 \\
3
\end{array}\right)} d_{2}^{\left(\begin{array}{c}
5 \\
2
\end{array}\right)} n^{4} \geq(1-3 \nu / 4) r d_{4}^{5} d_{3}^{\left(\begin{array}{c}
5 \\
3
\end{array}\right)} d_{2}^{\left(\begin{array}{c}
5 \\
2
\end{array}\right)} n^{4}
$$

copies of $K_{5}^{(4)}$, each of which uses exactly one vertex from $X^{(i)}=\left\{x_{1}^{(i)}, \ldots\right.$, $\left.x_{r}^{(i)}\right\}$.

Note that $\nu \gg \delta_{4}$ and, therefore, the following lower bound on the number of $K_{5}^{(4)}$, s in $\mathcal{H}^{(4)}$ holds:

$$
\begin{array}{r}
\left|\mathcal{K}_{5}\left(\mathcal{H}^{(4)}\right)\right| \geq(1-3 \nu / 4) r d_{4}^{5} d_{3}^{\left(\begin{array}{l}
5 \\
3
\end{array}\right)} d_{2}^{\left(\begin{array}{l}
2 \\
2
\end{array}\right)} n^{4} \times\left(1-100 \delta_{4}^{1 / 4}-\delta_{4}^{1 / 2}\right) \frac{n}{r} \\
\stackrel{(2.3 \mathrm{a})}{\geq}(1-\nu) d_{4}^{5} d_{3}^{\left(\begin{array}{l}
5 \\
3
\end{array}\right)} d_{2}^{\left(\begin{array}{c}
5 \\
2
\end{array}\right)} n^{5} .
\end{array}
$$


3.2. Upper bound. Due to the quantification of the Main Theorem, we can choose $\delta_{4}, \delta_{3}, \delta_{2}$, and $r$ in such a way that the lower bound derived in the previous subsection holds with $\nu$ replaced with $\nu d_{4}^{5} / 2$. In particular, we have

$$
\left|\mathcal{K}_{5}\left(\mathcal{H}^{(4)}\right)\right| \geq\left(1-\nu d_{4}^{5} / 2\right) d_{4}^{\left(\begin{array}{c}
5 \\
4
\end{array}\right)} d_{3}^{\left(\begin{array}{c}
5 \\
3
\end{array}\right)} d_{2}^{\left(\begin{array}{c}
5 \\
2
\end{array}\right)} n^{5}
$$

Using (1.3), we obtain

$$
\left|\mathcal{K}_{5}\left(\widetilde{\mathcal{H}}^{(4)}(\mathcal{G})\right)\right| \geq\left(1-\nu d_{4}^{5} / 2\right) d_{4}^{e(\mathcal{G})}\left(1-d_{4}\right)^{\left(\begin{array}{l}
5 \\
4
\end{array}\right)-e(\mathcal{G})} d_{3}^{\left(\begin{array}{c}
5 \\
3
\end{array}\right)} d_{2}^{\left(\begin{array}{c}
5 \\
2
\end{array}\right)} n^{5}
$$

for every subhypergraph $\mathcal{G}$ of $K_{5}^{(4)}$. Furthermore, notice that

$$
\mathcal{K}_{5}\left(\mathcal{H}^{(3)}\right)=\bigcup_{\mathcal{G} \subset K_{5}^{(4)}} \mathcal{K}_{5}\left(\widetilde{\mathcal{H}}^{(4)}(\mathcal{G})\right),
$$

and this union is disjoint because each $I \in \mathcal{K}_{5}\left(\mathcal{H}^{(3)}\right)$ induces exactly one subgraph $\mathcal{G}$ of $K_{5}^{(4)}$. Consequently

$$
\begin{array}{r}
\left|\mathcal{K}_{5}\left(\mathcal{H}^{(3)}\right)\right|=\sum_{\mathcal{G} \subset K_{5}^{(4)}}\left|\mathcal{K}_{5}\left(\widetilde{\mathcal{H}}^{(4)}(\mathcal{G})\right)\right|=\left|\mathcal{K}_{5}\left(\mathcal{H}^{(4)}\right)\right|+\sum_{\mathcal{G} \subsetneq K_{5}^{(4)}}\left|\mathcal{K}_{5}\left(\widetilde{\mathcal{H}}^{(4)}(\mathcal{G})\right)\right| \\
\stackrel{(3.15)}{\geq}\left|\mathcal{K}_{5}\left(\mathcal{H}^{(4)}\right)\right|+\left(1-\nu d_{4}^{5} / 2\right) \sum_{\mathcal{G} \subsetneq K_{5}^{(4)}} d_{4}^{e(\mathcal{G})}\left(1-d_{4}\right)^{\left(\begin{array}{l}
5 \\
4
\end{array}\right)-e(\mathcal{G})} d_{3}^{\left(\begin{array}{l}
5 \\
3
\end{array}\right)} d_{2}^{\left(\begin{array}{c}
5 \\
2
\end{array}\right)} n^{5} \\
=\left|\mathcal{K}_{5}\left(\mathcal{H}^{(4)}\right)\right|+\left(1-\nu d_{4}^{5} / 2\right)\left(1-d_{4}^{5}\right) d_{3}^{\left(\begin{array}{l}
5 \\
3
\end{array}\right)} d_{2}^{\left(\begin{array}{l}
5 \\
2
\end{array}\right)} n^{5} .
\end{array}
$$

On the other hand, we select $\delta_{4}, \delta_{3}, \delta_{2}$, and $r$ in such a way that the assumptions of Theorem 1.14 are satisfied with $\nu$ replaced with $\nu d_{4}^{5} / 2$. Then, we have

$$
\left|\mathcal{K}_{5}\left(\mathcal{H}^{(3)}\right)\right| \leq\left(1+\nu d_{4}^{5} / 2\right) d_{3}^{\left(\begin{array}{l}
5 \\
3
\end{array}\right)} d_{2}^{\left(\begin{array}{c}
5 \\
2
\end{array}\right)} n^{5}
$$

Comparing the last two inequalities yields

$$
\left|\mathcal{K}_{5}\left(\mathcal{H}^{(4)}\right)\right| \leq\left(\left(1+\nu d_{4}^{5} / 2\right)-\left(1-\nu d_{4}^{5} / 2\right)\left(1-d_{4}^{5}\right)\right) d_{3}^{\left(\begin{array}{c}
5 \\
3
\end{array}\right)} d_{2}^{\left(\begin{array}{c}
5 \\
2
\end{array}\right)} n^{5}
$$

One can easily observe that $\left(1+\nu d_{4}^{5} / 2\right)-\left(1-\nu d_{4}^{5} / 3\right)\left(1-d_{4}^{5}\right) \leq 1+\nu d_{4}^{5} / 2-$ $1+\nu d_{4}^{5} / 2+d_{4}^{5}=(1+\nu) d_{4}^{5}$. Consequently

$$
\left|\mathcal{K}_{5}\left(\mathcal{H}^{(4)}\right)\right| \leq(1+\nu) d_{4}^{\left(\begin{array}{c}
5 \\
4
\end{array}\right)} d_{3}^{\left(\begin{array}{l}
5 \\
3
\end{array}\right)} d_{2}^{\left(\begin{array}{l}
5 \\
2
\end{array}\right)} n^{5} .
$$

\section{More Definitions and facts about $(s, 2)$-Cylinders}

The main goal of this Chapter is to extend the notation from the Introduction and to provide some basic facts about $(s, 2)$-cylinders. We will also prove Propositions 2.2 and 2.3. Note that we will omit the elementary proofs of statements below and the reader familiar with applications of Szemerédi's Regularity Lemma is encouraged to skip this section entirely. 
Definition 4.1. Let $\mathcal{G}$ be an $(s, 2)$-cylinder with $s$-partition $U=U_{1} \cup \ldots \cup$ $U_{s}$. We define the neighborhood of a vertex $u \in U$ by $N(u)=N_{\mathcal{G}}(u)=\mathcal{G}(u)$ and the degree of $u$ by $\operatorname{deg}(u)=\operatorname{deg}_{\mathcal{G}}(u)=|\mathcal{G}(u)|$. If $W$ is a subset of vertices of $U$, we define $N(W)=N_{\mathcal{G}}(W)=\mathcal{G}(W)$ and $\operatorname{deg}(W)=\operatorname{deg}_{\mathcal{G}}(W)=$ $|\mathcal{G}(W)|$.

If $u \notin U_{j}, j \in[s]$, then we set $N_{j}(u)=N_{\mathcal{G}, j}(u)=N_{\mathcal{G}}(u) \cap U_{j}$ and $\operatorname{deg}_{j}(u)=\operatorname{deg}_{\mathcal{G}, j}(u)=\left|N_{\mathcal{G}, j}(u)\right|$. Similarly, if $W \cap U_{j}=\emptyset$, we set $N_{j}(W)=$ $N_{\mathcal{G}, j}(W)=N_{\mathcal{G}}(W) \cap U_{j}$ and $\operatorname{deg}_{j}(W)=\operatorname{deg}_{\mathcal{G}, j}(W)=\left|N_{\mathcal{G}, j}(W)\right|$.

Note that almost all of vertices in a regular $(2,2)$-cylinder have nearly the same degree. More precisely, the following fact is true:

Fact 4.2. Let $\mathcal{G}=\left(U_{1} \cup U_{2}, E\right)$ be a $(\delta, d)$-regular bipartite graph with $\left|U_{1}\right|=$ $\left|U_{2}\right|=m$ and $0<\delta \leq d$. Then $\operatorname{deg}(u)=(d \pm \delta) m$ for all but at most $2 \delta m$ vertices $u \in U_{1}$.

This fact extends to an arbitrary subset $W$ of vertices.

Fact 4.3. Let $k$ be a positive integer such that $(d-\delta)^{2 k} \geq \delta$, and let $\mathcal{G}$ be a $(\delta, d)$-regular $(s, 2)$-cylinder with s-partition $U_{1} \cup \ldots \cup U_{s},\left|U_{1}\right|=$ $\ldots=\left|U_{s}\right|=m$. Then, all but at most $2 k(s-1) \delta^{1 / 2} m^{k} k$-tuples of vertices $\left\{u_{1}, u_{2}, \ldots u_{k}\right\} \subseteq U_{1}$ satisfy the following condition:

For every $i \in[k]$ and every $j \in[s] \backslash\{1\}$, if $W$ is any subset of $\left\{u_{1}, u_{2}, \ldots u_{k}\right\}$, $|W|=i$, then

$$
\operatorname{deg}_{j}(W)=(d \pm \delta)^{i} m
$$

We will also use the following easy consequence of the definition of $(\delta, d)$ regularity.

Fact 4.4. Let $\mathcal{G}$ be a $(\delta, d)$-regular $(s, 2)$-cylinder with s-partition $U_{1} \cup \ldots \cup$ $U_{s}$, and let $U_{1}^{\prime} \subseteq U_{1}, U_{2}^{\prime} \subseteq U_{2}, \ldots, U_{k}^{\prime} \subseteq U_{k}$, be subsets such that $\left|U_{j}^{\prime}\right| \geq$ $\delta^{1 / 4} m$ for all $j \in[k]$. Let $\mathcal{G}^{\prime}$ be the subcylinder induced on $U_{1}^{\prime} \cup \ldots \cup U_{s}^{\prime}$. Then, $\mathcal{G}^{\prime}$ is $\left(\delta^{1 / 2}, d\right)$-regular.

Regular cylinders have the property that one can count the actual number of copies of small complete graphs. The precise statement is summarized in the following fact (see e.g. [NR03]):

Fact 4.5. For any positive integer $s$ and positive real numbers $d, \delta$ such that $\delta^{1 / 4} \leq(d-\delta)^{s-1}$, there exists a function $\theta_{s, d}(\delta), \theta_{s, d}(\delta) \rightarrow 0$ as $\delta \rightarrow 0$, such that whenever $\mathcal{G}$ is a $(\delta, d)$-regular $(s, 2)$-cylinder with $s$-partition $U_{1} \cup \ldots \cup U_{s}$, $\left|U_{1}\right|=\ldots=\left|U_{s}\right|=m$, then

$$
\left|\mathcal{K}_{s}(\mathcal{G})\right|=\left(1 \pm \theta_{s, d}(\delta)\right) m^{s} d^{\left(\begin{array}{c}
s \\
2
\end{array}\right)} .
$$

We will frequently use the following easy corollary of Fact 4.5.

Corollary 4.6. If $\delta$ is sufficiently small (i.e. $\delta \leq \delta(s, d)$ ), then

$$
(3 / 4) m^{s} d^{\left(\begin{array}{c}
s \\
2
\end{array}\right)} \leq\left|\mathcal{K}_{s}(\mathcal{G})\right| \leq(5 / 4) m^{s} d^{\left(\begin{array}{c}
s \\
2
\end{array}\right)} .
$$


Now we are ready to prove Propositions 2.2 and 2.3.

Proof of Proposition 2.2. By Fact 4.3 all but at most $8 \delta_{2}^{1 / 2} n$ vertices $x \in V_{1}$ satisfy

$$
\operatorname{deg}_{j}(x)=\left(d_{2} \pm \delta_{2}\right) n
$$

for $j=2,3,4,5$. For each such vertex, the $(4,2)$-cylinder $\mathcal{H}^{(2)}\left[\mathcal{H}^{(2)}(x)\right]$ is $\left(\delta_{2}^{1 / 2}, d_{2}\right)$-regular by Fact 4.4 . Since $\delta_{2} \ll d_{2}$ by assumption (2.3a), Corollary 4.6 implies that

$$
\left|\mathcal{K}_{4}\left(\mathcal{H}^{(2)}\left[\mathcal{H}^{(2)}(x)\right]\right)\right| \leq(5 / 4) d_{2}^{\left(\begin{array}{c}
4 \\
2
\end{array}\right)} \times\left(d_{2}+\delta_{2}\right)^{4} n^{4} \leq 2 d_{2}^{\left(\begin{array}{c}
5 \\
2
\end{array}\right)} n^{4} .
$$

Proof of Proposition 2.3. The proof follows the lines of the proof of Proposition 2.2 where we replace vertex $x$ satisfying $\operatorname{deg}_{j}(x)=\left(d_{2} \pm \delta_{2}\right) n$ for $j=2,3,4,5$, with a pair of vertices $\left\{x, x^{\prime}\right\}$ satisfying $\operatorname{deg}_{j}\left(x, x^{\prime}\right)=\left(d_{2} \pm \delta_{2}\right)^{2} n$ for $j=2,3,4,5$.

We now define the notion of a good vertex.

Definition 4.7 ( $\mathcal{G}$-good vertex). Let $\mathcal{G}$ be a $(\delta, d)$-regular $(s, 2)$-cylinder with $s$-partition $U_{1} \cup \ldots \cup U_{s},\left|U_{1}\right|=\ldots=\left|U_{s}\right|=m$. A vertex $u \in U_{1}$ is called $\mathcal{G}$-good if it satisfies

(i) $\operatorname{deg}_{j}(u)=(d \pm \delta) m$ for $j=2, \ldots, s$ and $(s-1,2)$-cylinder $\mathcal{G}[\mathcal{G}(u)]$ is $\left(\delta^{1 / 2}, d\right)$-regular,

(ii) $u$ extends to at most $\delta^{1 / 4} m$ pairs $\left\{u, u^{\prime}\right\} \subset U_{1}$ satisfying $\operatorname{deg}_{j}\left(u, u^{\prime}\right) \neq$ $(d \pm \delta)^{2} m$ for some $j \in\{2, \ldots, s\}$, or for which $(s-1,2)$-cylinder $\mathcal{G}\left[\mathcal{G}\left(u, u^{\prime}\right)\right]$ is not $\left(\delta^{1 / 2}, d\right)$-regular,

(iii) $u$ extends to at most $\delta^{1 / 4} m^{2}$ triples $\left\{u, u^{\prime}, u^{\prime \prime}\right\} \subset U_{1}$ not satisfying $\operatorname{deg}_{j}\left(u, u^{\prime}, u^{\prime \prime}\right)=(d \pm \delta)^{3} m$ for $j=2, \ldots, s$, or for which $(s-1,2)$ cylinder $\mathcal{G}\left[\mathcal{G}\left(u, u^{\prime}, u^{\prime \prime}\right)\right]$ is not $\left(\delta^{1 / 2}, d\right)$-regular.

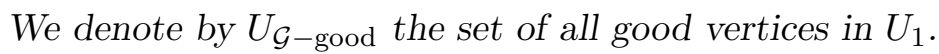

Suppose that $(d-\delta)^{3} \geq \delta^{1 / 4}$. Then for every vertex $u$ (pair $\left\{u, u^{\prime}\right\}$, triple $\left\{u, u^{\prime}, u^{\prime \prime}\right\}$, respectively) that satisfies condition (4.1), Fact 4.4 guarantees the regularity of $\mathcal{G}[\mathcal{G}(u)]\left(\mathcal{G}\left[\mathcal{G}\left(u, u^{\prime}\right)\right], \mathcal{G}\left[\mathcal{G}\left(u, u^{\prime}, u^{\prime \prime}\right)\right]\right.$, respectively).

It follows from Fact 4.3 that at most $2(s-1) \delta^{1 / 2} m$ vertices $u$, at most $4(s-1) \delta^{1 / 2} m^{2}$ pairs $\left\{u, u^{\prime}\right\}$, and at most $6(s-1) \delta^{1 / 2} m^{3}$ triples $\left\{u, u^{\prime}, u^{\prime \prime}\right\}$ violate condition (4.1). From this we can conclude that almost all vertices $u \in U_{1}$ are $\mathcal{G}$-good.

Observation 4.8. Let $\mathcal{G}$ be a $(\delta, d)$-regular $(s, 2)$-cylinder with s-partition $U_{1} \cup \ldots \cup U_{s},\left|U_{1}\right|=\ldots=\left|U_{s}\right|=m$, and $(d-\delta)^{3} \geq \delta^{1 / 4}$. Then

$$
\left|U_{\mathcal{G} \text {-good }}\right| \geq\left(1-2(s-1) \delta^{1 / 2}-10(s-1) \delta^{1 / 4}\right) m \geq\left(1-12(s-1) \delta^{1 / 4}\right) m \text {. }
$$

We next extend the notion of a good vertex to neighbors and pairs. 
Definition 4.9 ( $\mathcal{G}$-good neighbor). Let $\mathcal{G}$ be a $(\delta, d)$-regular $(s, 2)$-cylinder with $s$-partition $U_{1} \cup \ldots \cup U_{s},\left|U_{1}\right|=\ldots=\left|U_{s}\right|=m$, and $u \in U_{1}$ be a $\mathcal{G}$ good vertex. A vertex $v \in N_{\mathcal{G}, 2}(u)$ is called a $\mathcal{G}$-good neighbor if it is a

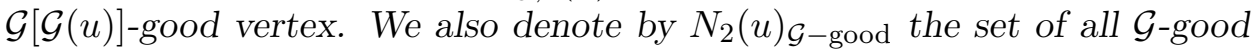
neighbors.

Note that for every $\mathcal{G}$-good neighbor $v \in N(u)_{\mathcal{G} \text {-good }}$ the graph $\mathcal{G}[\mathcal{G}(u, v)]$ is $\left(\delta^{1 / 4}, d\right)$-regular and $\operatorname{deg}_{j}(u, v)=\left(d \pm \delta^{1 / 2}\right)^{2} m$ holds for $j=3, \ldots, s$. One can observe the following:

Observation 4.10. Let $\mathcal{G}$ be a $(\delta, d)$-regular $(s, 2)$-cylinder with s-partition $U_{1} \cup \ldots \cup U_{s},\left|U_{1}\right|=\ldots=\left|U_{s}\right|=m$ and $\left(d-\delta^{1 / 2}\right)^{4} \geq \delta^{1 / 4}$. Then for every $\mathcal{G}$-good vertex $u \in U_{1}$ all but at most $12(s-2) \delta^{1 / 8}\left|N_{\mathcal{G}, 2}(u)\right|$ vertices $v \in N_{\mathcal{G}, 2}(u)$ are $\mathcal{G}$-good neighbors.

Definition 4.11 ( $\mathcal{G}$-good pair). Let $\mathcal{G}$ be a $(\delta, d)$-regular $(s, 2)$-cylinder with $s$-partition $U_{1} \cup \ldots \cup U_{s},\left|U_{1}\right|=\ldots=\left|U_{s}\right|=m$. A pair of $\mathcal{G}$-good vertices $\left\{u, u^{\prime}\right\} \subset U_{1}$ is called $\mathcal{G}$-good if it satisfies:

(i) $\operatorname{deg}_{j}\left(u, u^{\prime}\right)=(d \pm \delta)^{2} m$ for $j=2, \ldots, s$,

(ii) $\mathcal{G}\left[\mathcal{G}\left(u, u^{\prime}\right)\right]$ is $\left(\delta^{1 / 2}, d\right)$-regular,

(iii) $\left\{u, u^{\prime}\right\}$ extends to at most $\delta^{1 / 4} m$ sets $\left\{u, u^{\prime}, u^{\prime \prime}\right\} \subset U_{1}$ not satisfying $\operatorname{deg}_{j}\left(u, u^{\prime}, u^{\prime \prime}\right)=(d \pm \delta)^{3} m$ for $j=2, \ldots, s$, or for which $(s-1,2)$ cylinder $\mathcal{G}\left[\mathcal{G}\left(u, u^{\prime}, u^{\prime \prime}\right)\right]$ is not $\left(\delta^{1 / 2}, d\right)$-regular.

Similarly to the $\mathcal{G}$-good vertex case, almost all pairs of $\mathcal{G}$-good vertices are $\mathcal{G}$-good.

Observation 4.12. Let $0<\delta<d$ be two real numbers such that $(d-\delta)^{3} \geq$ $\delta^{1 / 4}$ and $\mathcal{G}$ be a $(\delta, d)$-regular $(s, 2)$-cylinder with s-partition $U_{1} \cup \ldots \cup U_{s}$, $\left|U_{1}\right|=\ldots=\left|U_{s}\right|=m$. Then all but at most $\left(4(s-1) \delta^{1 / 2}+6(s-1) \delta^{1 / 4}\right) m^{2}$ pairs in $\left[U_{\mathcal{G} \text {-good }}\right]^{2}$ are $\mathcal{G}$-good.

The proof of this Observation is similar to the proof of Observation 4.8.

\section{The $\ell$-GRAPHS LEMma}

The goal of this section is introduce the $\ell$-graphs Lemma which is the main tool in the proofs of Propositions 2.4 and 2.5. We start with some definitions and technical observations.

5.1. Definitions and technical observations. It is convenient to introduce the following notation: for a sequence of positive real numbers $\left\{d_{i}\right\}$, we set

$$
D_{t}=\prod_{i=1}^{t} d_{i} .
$$

Observe that $D_{t+1}=d_{t+1} \times D_{t}$.

The next definition is crucial for this part of the paper. 
Definition 5.1. Let $\mathcal{H}_{1}, \mathcal{H}_{2}$ be two $(k, k)$-cylinders with $k$-partition $U_{1} \cup$ $\ldots \cup U_{k}$, and $\mathcal{H}_{2} \subset \mathcal{H}_{1}$. We say that $\mathcal{H}_{2}$ is $(\varepsilon, d, r)$-regular with respect to $\mathcal{H}_{1}$ if the following is true: whenever $\mathcal{G}_{1}, \ldots, \mathcal{G}_{r}$ are $(k, k-1)$-cylinders with $k$-partition $U_{1} \cup \ldots \cup U_{k}$ such that

$$
\left|\mathcal{H}_{1} \cap \bigcup_{j=1}^{r} \mathcal{K}_{k}\left(\mathcal{G}_{j}\right)\right| \geq \varepsilon\left|\mathcal{H}_{1}\right|,
$$

then

$$
\left|\mathcal{H}_{2} \cap \bigcup_{j=1}^{r} \mathcal{K}_{k}\left(\mathcal{G}_{j}\right)\right|=(d \pm \varepsilon)\left|\mathcal{H}_{1} \cap \bigcup_{j=1}^{r} \mathcal{K}_{k}\left(\mathcal{G}_{j}\right)\right| .
$$

If $\mathcal{H}_{1}$ is the complete $(k, k)$-cylinder, then we simply say that $\mathcal{H}_{2}$ is $(\varepsilon, d, r)$ regular or, if $r=1,(\varepsilon, d)$-regular.

Note that if $\mathcal{H}_{2}$ is $(\varepsilon, d, r)$-regular with respect to $\mathcal{H}_{1}, \varepsilon^{\prime} \geq \varepsilon$, and $r^{\prime} \leq$ $r$, then $\mathcal{H}_{2}$ is also $\left(\varepsilon^{\prime}, d, r^{\prime}\right)$-regular with respect to $\mathcal{H}_{1}$. We will use this observation many times without mentioning it explicitly.

From now on we restrict ourselves to the graph case, i.e. $k=2$. One can observe that if $\mathcal{H}_{2}$ is $(\varepsilon, d, r)$-regular with respect to $\mathcal{H}_{1}$, then $\left|\mathcal{H}_{2}\right|=$ $(d \pm \varepsilon)\left|\mathcal{H}_{1}\right|$, and, more generally, that:

Observation 5.2. Let $\mathcal{H}_{1} \supset \mathcal{H}_{2} \supset \ldots \supset \mathcal{H}_{\ell}$ be bipartite graphs such that $\mathcal{H}_{i}$ is $\left(\varepsilon_{i}, d_{i}, r\right)$-regular with respect to $\mathcal{H}_{i-1}$ for all $i \in[\ell] \backslash\{1\}$. Then

$$
\prod_{j=2}^{\ell}\left(d_{j}-\varepsilon_{j}\right) \times\left|\mathcal{H}_{1}\right| \leq\left|\mathcal{H}_{\ell}\right| \leq \prod_{j=2}^{\ell}\left(d_{j}+\varepsilon_{j}\right) \times\left|\mathcal{H}_{1}\right| .
$$

Moreover, if $\mathcal{H}_{1}$ is $\left(\varepsilon_{1}, d_{1}\right)$-regular and $\left|U_{1}\right|=\left|U_{2}\right|=m$, then

$$
\prod_{j=1}^{\ell}\left(d_{j}-\varepsilon_{j}\right) \times m^{2} \leq\left|\mathcal{H}_{\ell}\right| \leq \prod_{j=1}^{\ell}\left(d_{j}+\varepsilon_{j}\right) \times m^{2} .
$$

We remark that the above observation is a density statement, i.e. the assumptions about regularity are not necessary and the statement remains true whenever the relative density of $\mathcal{H}_{i}$ with respect to $\mathcal{H}_{i-1}$ is $d_{i} \pm \varepsilon_{i}$ and the density of $\mathcal{H}_{1}$ is $d_{1} \pm \varepsilon_{1}$. We extend the above definition to the case of $(s, 2)$-cylinders.

Definition 5.3. Let $r \in \mathbb{N}$ and $\mathcal{H}_{1} \supset \mathcal{H}_{2}$ be two $(s, 2)$-cylinders. We say that $\mathcal{H}_{2}$ is $(\delta, d, r)$-regular with respect to $\mathcal{H}_{1}$ if $\mathcal{H}_{2}\left[U_{i} \cup U_{j}\right]$ is $(\delta, d, r)$-regular with respect to $\mathcal{H}_{1}\left[U_{i} \cup U_{j}\right]$ for all $1 \leq i<j \leq s$.

Having Definitions 5.1 and 5.3, we can present the statement of the $\ell$ graphs Lemma. First, we describe the scenario we are going to work with.

Setup A. Let $\mathcal{G}_{1}, \ldots, \mathcal{G}_{\ell}$ be $(s, 2)$-cylinders with $s$-partition $V=U_{1} \cup \ldots \cup U_{s}$, where $\left|U_{1}\right|=\ldots=\left|U_{s}\right|=m$, and such that the following conditions are satisfied:

(i) $\mathcal{G}_{i} \subset \mathcal{G}_{i-1}$ for all $i \in[\ell] \backslash\{1\}$, 
(ii) $0<\varepsilon_{i} \ll d_{i}<1$ for all $i \in[\ell]$,

(iii) $\varepsilon_{i-1}<\varepsilon_{i}$ for all $i \in[\ell] \backslash\{1\}$,

(iv) $\mathcal{G}_{1}$ is $\left(\varepsilon_{1}, d_{1}\right)$-regular and $\mathcal{G}_{i}$ is $\left(\varepsilon_{i}, d_{i}, r\right)$-regular with respect to $\mathcal{G}_{i-1}$ for all $i \in[\ell] \backslash\{1\}$

(v) $r \geq 2 \varepsilon_{\ell}^{1 / 2} \prod_{j=1}^{\ell} d_{j}^{-2}=2 \varepsilon_{\ell}^{1 / 2} D_{\ell}^{-2}$.

Then the $\ell$-graphs Lemma reads as follows.

Lemma 5.4 ( $\ell$-graphs Lemma). Suppose that $s=3$ and let $\ell \in \mathbb{N}$ be given. Then $\forall d_{\ell} \in(0,1] \exists \varepsilon_{\ell} \forall d_{\ell-1} \in(0,1] \exists \varepsilon_{\ell-1} \ldots \forall d_{1} \in(0,1] \exists \varepsilon_{1} \exists r$ so that if Setup A holds, then

$$
\left|\mathcal{K}_{3}\left(\mathcal{G}_{\ell}\right)\right|=\left(1 \pm \varepsilon_{\ell}^{1 / 64}\right) D_{\ell}^{3} m^{3} .
$$

The proof of this lemma follows from Facts (5.5) and (5.6) by induction on $\ell$. Details are in [Sk00, RS04b] and we omit them here. For $\ell=2$, this lemma was proved in a slightly different setting in [FR02].

Fact 5.5. For every $i \in[\ell]$, all but at most $4(s-1)\left(\varepsilon_{1}^{1 / 2}+\ldots+\varepsilon_{i}^{1 / 2}\right) m$ vertices $u \in U_{1}$ satisfy

$$
\operatorname{deg}_{\mathcal{G}_{j}, \alpha}(u)=\left(1 \pm \varepsilon_{j}^{1 / 2}\right)^{j} D_{j} m
$$

for all $j \in[i]$ and $\alpha \in[s] \backslash\{1\}$.

Fact 5.6. For every $i \in[\ell]$, all but at most $(s-1) \sum_{j=1}^{i}\left(4 \varepsilon_{j}^{1 / 2}+8 \varepsilon_{j}^{1 / 8}\right) m^{2}$ pairs of vertices $u, u^{\prime} \in U_{1}$ satisfy

(i) $u$ and $u^{\prime}$ satisfy (5.4), and

(ii) $\operatorname{deg}_{\mathcal{G}_{j}, \alpha}\left(u, u^{\prime}\right)=\left|N_{\mathcal{G}_{j}}\left(u, u^{\prime}\right) \cap U_{\alpha}\right|=\left(1 \pm \varepsilon_{j}^{1 / 8}\right)^{j} D_{j}^{2} m$ for all $j \in[i]$ and $\alpha \in[s] \backslash\{1\}$.

In the following two sections, we investigate link properties of a regular $(s, 3)$-cylinders in two distinct setups. Since these properties are later used in several different settings, we use a different notation now and later explain transitions between setups and a particular setting.

\section{Properties of Links of 3-Cylinders}

The aim of this section is to present several auxiliary statements regarding the properties of links in 3-uniform hypergraphs. We state these statements without a proof since similar facts have already appeared in the literature. For the full proofs of the claims below the reader is encouraged to see [Sk00] or cited sources. We consider the following setup:

Setup B. Let $0<\varepsilon_{2} \ll d_{2} \leq 1$ and $0<\varepsilon_{3} \ll d_{3} \leq 1$ be real numbers so that $\varepsilon_{2} \ll \varepsilon_{3}$. Let $U=U_{1} \cup \ldots \cup U_{s}$ be a partition, where $\left|U_{1}\right|=\ldots=$ $\left|U_{s}\right|=m, \mathcal{G}=(U, E(\mathcal{G}))$ be an $(s, 2)$-cylinder that is $\left(\varepsilon_{2}, d_{2}\right)$-regular, and let $\mathcal{H}=(U, E(\mathcal{H}))$ be an $(s, 3)$-cylinder which is $\left(\varepsilon_{3}, d_{3}, r\right)$-regular with respect to $\mathcal{G}$. 
The first claim shows that the link $\mathcal{H}(u)$ is $\left(2 \varepsilon_{3}^{1 / 2}, d_{3}, r\right)$-regular for almost all good vertices $u$. A similar fact has been proved in [FR02] (as Claim A) and in [NR03] (as Fact 4.0.23).

Claim 6.1. The link $\mathcal{H}(u)$ is $\left(2 \varepsilon_{3}^{1 / 2}, d_{3}, r\right)$-regular with respect to $\mathcal{G}[\mathcal{G}(u)]$ (i.e. with respect to the subgraph of $\mathcal{G}$ induced on $\mathcal{G}(u)$ ) for all but at most $4\left(\begin{array}{c}s-1 \\ 2\end{array}\right) \varepsilon_{3}^{1 / 2} m \mathcal{G}$-good vertices $u \in U_{1}$.

The next claim shows that the restriction of $\mathcal{H}$ to the neighborhood $\mathcal{G}(u)$ of $u$ is $\left(2 \varepsilon_{3}^{1 / 4}, d_{3}, r^{\prime}\right)$-regular (note that $r^{\prime}<r$ in this case) for almost all $\mathcal{G}$-good vertices $u$. A similar claim has been proved in [PRS04] (as Claim $6.2)$.

Claim 6.2. Let $t=\varepsilon_{3}^{1 / 2} d_{2}^{-3}$ and $r^{\prime}=r / t$. Then $(s-1,3)$-cylinder $\mathcal{H}$ is $\left(2 \varepsilon_{3}^{1 / 4}, d_{3}, r^{\prime}\right)$-regular with respect to $\mathcal{G}[\mathcal{G}(u)]$ for all but at most $2\left(\begin{array}{c}s-1 \\ 3\end{array}\right) \varepsilon_{2}^{1 / 16} m$ $\mathcal{G}$-good vertices $u \in U_{1}$.

In order to distinguish vertices that satisfy both previous claims, we call them $\mathcal{H}$-nice.

Definition 6.3 ( $\mathcal{H}$-nice vertex). A vertex $u \in U_{\mathcal{G} \text {-good }}$ is called $\mathcal{H}$-nice if it satisfies:

(i) $\mathcal{H}(u)$ is $\left(2 \varepsilon_{3}^{1 / 2}, d_{3}, r\right)$-regular with respect to $\mathcal{G}[\mathcal{G}(u)]$, and

(ii) $\mathcal{H}$ is $\left(2 \varepsilon_{3}^{1 / 4}, d_{3}, r /\left(\varepsilon_{3}^{1 / 2} d_{2}^{-3}\right)\right)$-regular with respect to $\mathcal{G}[\mathcal{G}(u)]$.

We denote by $U_{\mathcal{H} \text {-nice }}$ the set of all nice vertices in $U_{1}$.

Claims 6.1 and 6.2 imply the following observation:

Observation 6.4. All but at most $4\left(\begin{array}{c}s-1 \\ 3\end{array}\right) \varepsilon_{3}^{1 / 2} m+2\left(\begin{array}{c}s-1 \\ 2\end{array}\right) \varepsilon_{2}^{1 / 16} m \leq 6\left(\begin{array}{c}s-1 \\ 3\end{array}\right) \varepsilon_{3}^{1 / 2} m$ $\mathcal{G}$-good vertices are $\mathcal{H}$-nice, i.e.

$$
\left|U_{\mathcal{H}-\text { nice }}\right| \geq\left|U_{\mathcal{G} \text {-good }}\right|-6\left(\begin{array}{c}
s-1 \\
3
\end{array}\right) \varepsilon_{3}^{1 / 2} m .
$$

The following claim is an extension of Claim 6.1 to pairs of vertices. Note that a similar claim is proved (as Lemma 3.4) in [DHNR02].

Claim 6.5. Let $t=\left(\varepsilon_{3}^{1 / 2} d_{2}^{-3}\right)\left(2 \varepsilon_{3}^{1 / 8} d_{2}^{-2}\right)$ and $r^{\prime}=r / t$. Then for all but most $2\left(\begin{array}{c}s-1 \\ 2\end{array}\right) \varepsilon_{2}^{1 / 16} m \mathcal{H}$-nice vertices $u \in U_{1}$ the following statement is true:

The link $\mathcal{H}\left(u, u^{\prime}\right)$ is $\left(\varepsilon_{3}^{1 / 32}, d_{3}^{2}, r^{\prime}\right)$-regular with respect to $\mathcal{G}\left[\mathcal{G}\left(u, u^{\prime}\right)\right]$ (i.e. with respect to the subgraph of $\mathcal{G}$ induced on $\mathcal{G}\left(u, u^{\prime}\right)$ ) for all but at most $5\left(\begin{array}{c}s-1 \\ 2\end{array}\right) \varepsilon_{3}^{1 / 16} m \mathcal{G}$-good pairs $\left\{u, u^{\prime}\right\}$, where $u^{\prime} \in U_{\mathcal{H}-\text { nice }}$.

The next claim, that is similar to Claim 6.2 in [PRS04], extends Claim 6.2 to pairs.

Claim 6.6. Let $t=\left(\varepsilon_{3}^{1 / 2} d_{2}^{-3}\right)\left(2 \varepsilon_{3}^{1 / 8} d_{2}^{-2}\right)$ and $r^{\prime}=r / t$. Then, for every $\mathcal{H}$ nice vertex $u \in U_{1}$, the restriction of $\mathcal{H}$ to $\mathcal{G}\left(u, u^{\prime}\right)$ is $\left(4 \varepsilon_{3}^{1 / 16}, d_{3}, r^{\prime}\right)$-regular 
with respect to $\mathcal{G}\left[\mathcal{G}\left(u, u^{\prime}\right)\right]$ for all but at most $3\left(\begin{array}{c}s-1 \\ 3\end{array}\right) \varepsilon_{2}^{1 / 32} m \mathcal{G}$-good vertices $u^{\prime} \in U_{1}$.

We call all pairs of vertices $\left\{u, u^{\prime}\right\}$ satisfying the above claims $\mathcal{H}$-nice.

Definition $6.7\left(\mathcal{H}\right.$-nice pair). Let $t=\left(\varepsilon_{3}^{1 / 2} d_{2}^{-3}\right)\left(2 \varepsilon_{3}^{1 / 8} d_{2}^{-2}\right)$ and $r^{\prime}=r / t$. A pair of vertices $\left\{u, u^{\prime}\right\}$ is called $\mathcal{H}$-nice if it satisfies the following conditions:

(i) both $u$ and $u^{\prime}$ are $\mathcal{H}$-nice vertices,

(ii) the pair $\left\{u, u^{\prime}\right\}$ is $\mathcal{G}$-good,

(iii) the link $\mathcal{H}\left(u, u^{\prime}\right)$ is $\left(\varepsilon_{3}^{1 / 32}, d_{3}^{2}, r^{\prime}\right)$-regular with respect to the subgraph of $\mathcal{G}$ induced on $\mathcal{G}\left(u, u^{\prime}\right)$, and

(iv) the restriction of $\mathcal{H}$ to $\mathcal{G}\left(u, u^{\prime}\right)$ is $\left(4 \varepsilon_{3}^{1 / 16}, d_{3}, r^{\prime}\right)$-regular with respect to the subgraph of $\mathcal{G}$ induced on $\mathcal{G}\left(u, u^{\prime}\right)$.

Observations 4.8, 4.12, and 6.4, and Claims 6.6 and 6.5 imply the following observation (we use $\varepsilon_{2} \ll \varepsilon_{3}$ to simplify this result).

Observation 6.8. All but $3\left(\begin{array}{c}s-1 \\ 2\end{array}\right) \varepsilon_{3}^{1 / 16} m^{2}$ pairs in $\left[U_{1}\right]^{2}$ are $\mathcal{H}$-nice.

\section{Counting in 3-Cylinders}

In this section, we are going to work with the following setup:

Setup C. Let $0<\varepsilon_{2} \ll d_{2} \leq 1,0<\varepsilon_{3} \ll d_{3} \leq 1$, and $0<\varepsilon_{3} \ll \tilde{d}_{3} \leq 1$ be real numbers so that $\varepsilon_{2} \ll \varepsilon_{3}$. Let $U=U_{1} \cup \ldots \cup U_{s}$ be a partition, where $\left|U_{1}\right|=\ldots=\left|U_{s}\right|=m, \mathcal{G}_{2}=\left(U, E\left(\mathcal{G}_{2}\right)\right)$ be an $(s, 2)$-cylinder that is $\left(\varepsilon_{2}, d_{2}\right)$-regular, $\mathcal{G}_{3}=\left(U, E\left(\mathcal{G}_{3}\right)\right)$ be an $(s, 2)$-cylinder that is $\left(\varepsilon_{3}, \tilde{d}_{3}, r\right)$ regular with respect to $\mathcal{G}_{2}$, and let $\mathcal{H}=(U, E(\mathcal{H}))$ be an $(s, 3)$-cylinder which is $\left(\varepsilon_{3}, d_{3}, r\right)$-regular with respect to $\mathcal{G}_{2}$.

Our goal is to prove the following counting claim that is later used in the proof of Proposition 2.4.

Claim 7.1. Let $s=4$ and $\mu>0$ be given. Then for $\mathcal{G}_{2}, \mathcal{G}_{3}$, and $\mathcal{H}$ as in Setup $C$ and $\varepsilon_{3} \ll \mu$, we have

$$
\left|\mathcal{K}_{4}\left(\mathcal{H} \cap \mathcal{K}_{3}\left(\mathcal{G}_{3}\right)\right)\right|=(1 \pm \mu) d_{2}^{6} \tilde{d}_{3}^{6} d_{3}^{4} m^{4} .
$$

In the proof of this claim, we will need the following two claims regarding the link properties of $\mathcal{G}_{2}, \mathcal{G}_{3}$, and $\mathcal{H}$. The first claim shows that $\mathcal{H}$ is regular with respect to $\mathcal{G}_{2}\left[\mathcal{G}_{2}(u)\right]$ for almost all good vertices $u$.

Claim 7.2. Let $t=\varepsilon_{3}^{1 / 2} d_{2}^{-3}$ and $r^{\prime}=r / t$. Then $\mathcal{H}$ is $\left(2 \varepsilon_{3}^{1 / 4}, d_{3}, r^{\prime}\right)$ regular with respect to $\mathcal{G}_{2}\left[\mathcal{G}_{2}(u)\right]$ for all but at most $2\left({ }^{s-1}{ }_{3}\right) \varepsilon_{2}^{1 / 16} m$ vertices $u \in U_{\mathcal{G}_{2} \text {-good }}$.

This claim is an immediate consequence of Claim 6.2.

The next claim shows that the 2-cylinder $\mathcal{G}_{3} \cap \mathcal{H}(u)$ is regular with respect to $\mathcal{G}_{2}$ for almost all $\mathcal{G}_{2}$-good vertices $u$. 
Claim 7.3. Let $r^{\prime}=r /\left(\varepsilon_{3}^{1 / 2} d_{2}^{-2}\right)$. Then,

(a) $\operatorname{deg}_{\mathcal{G}_{3}, j}(u)=\left(1 \pm \varepsilon_{3}^{1 / 2}\right)^{2} d_{2} \tilde{d}_{3} m$ for every $j \in[s] \backslash\{1\}$,

(b) $\mathcal{G}_{2}\left[\mathcal{G}_{3}(u)\right]$ is $\left(\varepsilon_{2}^{1 / 2}, d_{2}\right)$-regular, and

(c) $(s-1,2)$-cylinder $\mathcal{G}_{3}\left[\mathcal{G}_{3}(u)\right] \cap \mathcal{H}(u)$ is $\left(\varepsilon_{3}^{1 / 8}, d_{3} \tilde{d}_{3}, r^{\prime}\right)$-regular with respect to $\mathcal{G}_{2}\left[\mathcal{G}_{3}(u)\right]$ for all but at most $3\left(\begin{array}{c}s-1 \\ 2\end{array}\right) \varepsilon_{3}^{1 / 8} m \mathcal{G}_{2}$-good vertices $u \in U_{1}$.

Part (a) follows from Fact 5.5 and part $(b)$ is a consequence of the definition of $(\delta, d)$-regularity (cf. Definition 1.9). The proof of $(c)$ is analoguous to the proof of Claim 6.5 and we omit it here.

Proof of Claim 7.1. Let $\mu>0$ be given, let $r^{\prime}=r /\left(\varepsilon_{3}^{1 / 2} d_{2}^{-3}\right)$, and denote by $W$ the set of all $\mathcal{G}_{2}$-good vertices $u \in U_{1}$ for which

(i) $\mathcal{H}\left[\mathcal{G}_{2}(u)\right]$ is $\left(2 \varepsilon_{3}^{1 / 4}, d_{3}, r^{\prime}\right)$-regular with respect to $\mathcal{G}_{2}\left[\mathcal{G}_{2}(u)\right]$,

(ii) $\operatorname{deg}_{\mathcal{G}_{3}, j}(u)=\left(1 \pm \varepsilon_{3}^{1 / 2}\right)^{2} d_{2} \tilde{d}_{3} m$ for $j=2,3,4$, and

(iii) $\mathcal{G}_{3}\left[\mathcal{G}_{3}(u)\right] \cap \mathcal{H}(u)$ is $\left(\varepsilon_{3}^{1 / 8}, d_{3} \tilde{d}_{3}, r^{\prime}\right)$-regular with respect to $\mathcal{G}_{2}\left[\mathcal{G}_{3}(u)\right]$.

Furthermore, the $\mathcal{G}_{2}$-goodness of every vertex $u \in W$ implies

(iv) $\mathcal{G}_{2}\left[\mathcal{G}_{2}(u)\right]$ and $\mathcal{G}_{2}\left[\mathcal{G}_{3}(u)\right]$ are $\left(\varepsilon_{2}^{1 / 2}, d_{2}\right)$-regular and $\left(d_{2} \pm \varepsilon_{2}\right) m$ for all $j=2,3,4$.

It follows from Claims 7.2 and 7.3 that all but at most $2 \varepsilon_{2}^{1 / 16} m$ vertices

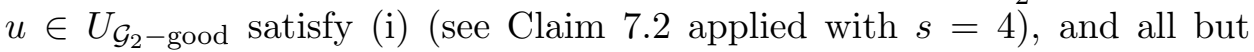

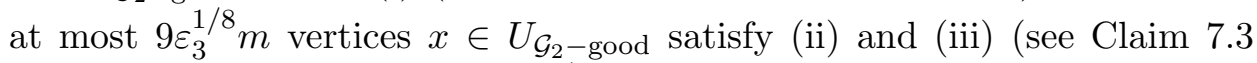
applied with $s=4$ and $\left.r^{\prime}<r /\left(\varepsilon_{3}^{1 / 2} d_{2}^{-2}\right)\right)$. We use assumptions $\varepsilon_{2} \ll \varepsilon_{3} \ll 1$ and obtain

$$
|W| \geq\left|U_{\mathcal{G}_{2}-\text { good }}\right|-10 \varepsilon_{3}^{1 / 8} m .
$$

Moreover, the size of $U_{\mathcal{G}_{2} \text {-good }}$ is bounded from below by Observation 4.8 applied with $s=4$ and $\delta=\varepsilon_{2}$ :

$$
\left|U_{\mathcal{G}_{2}-\text { good }}\right| \geq\left(1-36 \varepsilon_{2}^{1 / 4}\right) m \text {. }
$$

We proceed as follows: for every vertex $u \in W$ we apply the 2-graphs Lemma on $(3,2)$-cylinders $\mathcal{G}_{2}\left[\mathcal{G}_{3}(u)\right]$ and $\mathcal{H}(u) \cap \mathcal{G}_{3}\left[\mathcal{G}_{3}(u)\right]$. We will show that the number of copies of $K_{3}$ in $\mathcal{H}(u) \cap \mathcal{G}_{3}\left[\mathcal{G}_{3}(u)\right]$ is sufficiently large to apply the regularity of $\mathcal{H}\left[\mathcal{G}_{2}(u)\right]$ with respect to $\mathcal{G}_{2}\left[\mathcal{G}_{2}(u)\right]$. This way we will be able to count the number of edges in $\mathcal{H}$ which are also copies of $K_{3}$ in $\mathcal{H}(u) \cap \mathcal{G}_{3}\left[\mathcal{G}_{3}(u)\right]$. Notice that every such an edge together with $u$ form a copy of $K_{4}^{(3)}$ in $\mathcal{H} \cap \mathcal{K}_{3}\left(\mathcal{G}_{3}\right)$ that uses $u$ as a vertex. Then we add these numbers through all $u \in W$. Finally, we estimate the number of copies of $K_{4}^{(3)}$ in $\mathcal{H} \cap \mathcal{K}_{3}\left(\mathcal{G}_{3}\right)$ that use vertices not belonging to $W$.

Consider an arbitrary vertex $u \in W$. We apply the 2-graphs Lemma with

- $(3,2)$-cylinder $\mathcal{G}_{1}$ replaced by $\mathcal{G}_{2}\left[\mathcal{G}_{3}(u)\right]$ that is $\left(\varepsilon_{2}^{1 / 2}, d_{2}\right)$-regular (see (iv)); 
- $(3,2)$-cylinder $\mathcal{G}_{2}$ replaced by $\mathcal{G}_{3}\left[\mathcal{G}_{3}(u)\right] \cap \mathcal{H}(u)$ that is $\left(\varepsilon_{3}^{1 / 8}, d_{3} \tilde{d}_{3}, r^{\prime}\right)$ regular with respect to $\mathcal{G}_{2}\left[\mathcal{G}_{3}(u)\right]$ (see (iii));

- $U_{i}$ replaced by $\mathcal{G}_{3}(u) \cap U_{i+1}$ for $i=1,2,3$;

and obtain $\left|\mathcal{K}_{3}\left(\mathcal{H}(u) \cap \mathcal{G}_{3}\left[\mathcal{G}_{3}(u)\right]\right)\right|=\left(1 \pm \varepsilon_{3}^{1 / 512}\right)\left(d_{2} d_{3} \tilde{d}_{3}\right)^{3}\left(\left(1 \pm \varepsilon_{3}^{1 / 2}\right)^{2} d_{2} \tilde{d}_{3} m\right)^{3}$. This can be further simplified using the assumption $\varepsilon_{3} \ll \mu$ :

$$
\left|\mathcal{K}_{3}\left(\mathcal{H}(u) \cap \mathcal{G}_{3}\left[\mathcal{G}_{3}(u)\right]\right)\right|=(1 \pm \mu / 4) d_{2}^{6} \tilde{d}_{3}^{6} d_{3}^{3} m^{3} .
$$

Since $\mathcal{G}_{2}\left[\mathcal{G}_{2}(u)\right]$ is $\left(\varepsilon_{2}^{1 / 2}, d_{2}\right)$-regular, by Corollary $4.6,\left|\mathcal{K}_{3}\left(\mathcal{G}_{2}\left[\mathcal{G}_{2}(u)\right]\right)\right| \leq$ $(5 / 4) d_{2}^{3} \times\left(d_{2}+\varepsilon_{2}\right)^{3} m^{3} \leq(3 / 2) d_{2}^{6} m^{3}$. As $d_{3}, \tilde{d}_{3} \gg \varepsilon_{3}$, we obtain

$$
\begin{aligned}
2 \varepsilon_{3}^{1 / 4}\left|\mathcal{K}_{3}\left(\mathcal{G}_{2}\left[\mathcal{G}_{2}(u)\right]\right)\right| \leq & 2 \varepsilon_{3}^{1 / 4} \times \frac{3}{2} d_{2}^{6} m^{3} \\
& \leq(1-\mu / 4) d_{2}^{6} \tilde{d}_{3}^{6} d_{3}^{3} m^{3} \leq\left|\mathcal{K}_{3}\left(\mathcal{H}(u) \cap \mathcal{G}_{3}\left[\mathcal{G}_{3}(u)\right]\right)\right|
\end{aligned}
$$

Thus, we can use the $\left(2 \varepsilon_{3}^{1 / 4}, d_{3}, r^{\prime}\right)$-regularity of $\mathcal{H}\left[\mathcal{G}_{2}(u)\right]$ with respect to $\mathcal{G}_{2}\left[\mathcal{G}_{2}(u)\right]$ (cf. (i)) and obtain

$$
\left|\mathcal{H} \cap \mathcal{K}_{3}\left(\mathcal{H}(u) \cap \mathcal{G}_{3}\left[\mathcal{G}_{3}(u)\right]\right)\right|=\left(d_{3} \pm 2 \varepsilon_{3}^{1 / 4}\right)\left|\mathcal{K}_{3}\left(\mathcal{H}(u) \cap \mathcal{G}_{3}\left[\mathcal{G}_{3}(u)\right]\right)\right| .
$$

Combining (7.1) and (7.2) yields

$$
\left|\mathcal{H} \cap \mathcal{K}_{3}\left(\mathcal{H}(u) \cap \mathcal{G}_{3}\left[\mathcal{G}_{3}(u)\right]\right)\right|=(1 \pm \mu / 2) d_{2}^{6} \tilde{d}_{3}^{6} d_{3}^{4} m^{3} .
$$

As mentioned before, every edge in $\mathcal{H}$ which is also a copy of $K_{3}$ in $\mathcal{H}(u) \cap \mathcal{G}_{3}\left[\mathcal{G}_{3}(u)\right]$ forms together with $u$ a copy of $K_{4}^{(3)}$ in $\mathcal{H} \cap \mathcal{K}_{3}\left(\mathcal{G}_{3}\right)$ that uses $u$ as a vertex. Therefore, there is at least $|W| \times(1-\mu / 2) d_{2}^{6} \tilde{d}_{3}^{6} d_{3}^{4} m^{3}$ copies of $K_{4}^{(3)}$ in $\mathcal{H} \cap \mathcal{K}_{3}\left(\mathcal{G}_{3}\right)$.

Since the size of $W$ is at least $\left|U_{\mathcal{G}_{2} \text {-good }}\right|-10 \varepsilon_{3}^{1 / 8} m \geq\left(1-36 \varepsilon_{3}^{1 / 4}-\right.$ $\left.10 \varepsilon_{3}^{1 / 8}\right) m \geq\left(1-11 \varepsilon_{3}^{1 / 8}\right) m$, the following lower bound holds:

$$
\left|\mathcal{K}_{4}\left(\mathcal{H} \cap \mathcal{K}_{3}\left(\mathcal{G}_{3}\right)\right)\right| \geq\left(1-11 \varepsilon_{3}^{1 / 8}\right) m \times(1-\mu / 2) d_{2}^{6} \tilde{d}_{3}^{6} d_{3}^{4} m^{3} \geq(1-\mu) d_{2}^{6} \tilde{d}_{3}^{6} d_{3}^{4} m^{4} .
$$

Similarly as above, the contribution of vertices from $W$ is bounded by $(1+\mu / 2) d_{2}^{6} \tilde{d}_{3}^{6} d_{3}^{4} m^{4}$ and a similar calculation shows that vertices in $U_{1} \backslash W$ are in at most $(\mu / 2) d_{2}^{6} \tilde{d}_{3}^{6} d_{3}^{4} m^{4}$ copies of $K_{4}^{(3)}$.

\section{Proof of Proposition 2.4}

Recall that our goal is to prove Proposition 2.4, i.e. we want to bound the number of copies of $K_{4}^{(3)}$ in $\mathcal{H}^{(3)} \cap \mathcal{K}_{3}\left(\mathcal{H}^{(3)}\left(x, x^{\prime}\right)\right)$ for almost all pairs of vertices $x, x^{\prime} \in V_{1}$. We fix an $\mathcal{H}^{(3)}$-nice pair of vertices $x, x^{\prime} \in V_{1}$ (cf. Definition 6.7 applied with $\mathcal{G}=\mathcal{H}^{(2)}, \mathcal{H}=\mathcal{H}^{(3)}, \varepsilon_{2}=\delta_{2}$, and $\left.\varepsilon_{3}=\delta_{3}\right)$ and set $r^{\prime}=r /\left(\delta_{3}^{1 / 2} d_{2}^{-3}\right)\left(2 \delta_{3}^{1 / 8} d_{2}^{-3}\right)$. Then, (ii), (iii), and (iv) of Definition 6.7 holds:

(ii) $\left\{x, x^{\prime}\right\}$ is $\mathcal{H}^{(2)}$-good, 
(iii) the link $\mathcal{H}^{(3)}\left(x, x^{\prime}\right)$ is $\left(\delta_{3}^{1 / 32}, d_{3}^{2}, r^{\prime}\right)$-regular with respect to the graph $\mathcal{H}^{(2)}\left[\mathcal{H}^{(2)}\left(x, x^{\prime}\right)\right]$,

(iv) $\mathcal{H}^{(3)}\left[\mathcal{H}^{(2)}\left(x, x^{\prime}\right)\right]$ is $\left(4 \delta_{3}^{1 / 16}, d_{3}, r^{\prime}\right)$-regular with respect to the graph $\mathcal{H}^{(2)}\left[\mathcal{H}^{(2)}\left(x, x^{\prime}\right)\right]$.

Since $\left\{x, x^{\prime}\right\}$ is also an $\mathcal{H}^{(2)}$-good pair (by (ii)), we have (see Definition 4.11)

(ii') $\operatorname{deg}_{j}\left(x, x^{\prime}\right)=\left(d_{2} \pm \delta_{2}\right)^{2} n$ for $j=2, \ldots, 5$, and

(ii") the $(4,2)$-cylinder $\mathcal{H}^{(2)}\left[\mathcal{H}^{(2)}\left(x, x^{\prime}\right)\right]$ is $\left(\delta_{2}^{1 / 2}, d_{2}\right)$-regular.

We apply Claim 7.1 with 4-partition $U=U_{1} \cup U_{2} \cup U_{3} \cup U_{4}$, where $U_{i}=V_{i+1} \cap \mathcal{H}^{(2)}\left(x, x^{\prime}\right)$ for $i \in[4]$, cylinders $\mathcal{G}_{2}=\mathcal{H}^{(2)}\left[\mathcal{H}^{(2)}\left(x, x^{\prime}\right)\right], \mathcal{G}_{3}=$ $\mathcal{H}^{(3)}\left(x, x^{\prime}\right)$, and $\mathcal{H}=\mathcal{H}^{(3)}\left[\mathcal{H}^{(2)}\left(x, x^{\prime}\right)\right]$, and parameters $\mu=1 / 2, \varepsilon_{2}=\delta_{2}^{1 / 2}$, $\varepsilon_{3}=4 \delta_{3}^{1 / 16}<\delta_{3}^{1 / 32}, \tilde{d}_{3}=d_{3}^{2}, m=\left(d_{2} \pm \delta_{2}\right)^{2} n$, and $r$ replaced by $r^{\prime}$. Observe that (ii'), (ii"), (iii), and (iv) verify conditions of the Setup C. Hence, we obtain

$$
\left|\mathcal{K}_{4}\left(\mathcal{H} \cap \mathcal{K}_{3}\left(\mathcal{G}_{3}\right)\right)\right| \leq \frac{3}{2} d_{2}^{6}\left(d_{3}^{2}\right)^{6} d_{3}^{4}\left(d_{2}+\delta_{2}\right)^{8} n^{4} .
$$

Estimate (2.6) follows from this inequality since $d_{2} \gg \delta_{2}$. This is true for every $\mathcal{H}^{(3)}$-nice pair $\left\{x, x^{\prime}\right\}$. By Observation 6.8 applied with $s=5$, all but at most $60 \delta_{3}^{1 / 16} n^{2}$ pairs $\left\{x, x^{\prime}\right\}$ are $\mathcal{H}^{(3)}$-nice. Therefore, (2.6) holds for all but at most $60 \delta_{3}^{1 / 16} n^{2}$ pairs $\left\{x, x^{\prime}\right\} \subset V_{1}$.

\section{NiCE NEIGHBORS}

The motivation for this subsection is twofold. First, we define the notion of an $\mathcal{H}$-nice neighbor and prove that almost all neighbors of an $\mathcal{H}$-nice vertex are $\mathcal{H}$-nice. Second, in the proof of Proposition 2.5, we will need an upper bound on the number of edges in $\mathcal{H}$ that are triangles in the joint neighborhood of two $\mathcal{H}$-nice neighbors. We provide this estimate at the end of this section. We start with the definition of an $\mathcal{H}$-nice neighbor.

Suppose that $\mathcal{G}$ and $\mathcal{H}$ are as in Setup B, i.e. $\mathcal{G}=(V, E(\mathcal{G}))$ is an $\left(\varepsilon_{2}, d_{2}\right)$ regular $(s, 2)$-cylinder and $\mathcal{H}=(V, E(\mathcal{H}))$ an $(s, 3)$-cylinder which is $\left(\varepsilon_{3}, d_{3}, r\right)$ regular with respect to $\mathcal{G}$.

Definition 9.1 ( $\mathcal{H}$-nice neighbor). Let $u \in U_{1}$ be an $\mathcal{H}$-nice vertex and set $r^{\prime}=r /\left(\varepsilon_{3}^{1 / 2} d_{2}^{-3}\right)\left(2 \varepsilon_{3}^{1 / 8} d_{2}^{-3}\right)$. A $\mathcal{G}$-good neighbor $v \in N_{2}(u)_{\mathcal{G} \text {-good }}$ is called $\mathcal{H}$-nice if

(i) $\operatorname{deg}_{\mathcal{H}(u), j}(v)=\left(1 \pm 2 \varepsilon_{3}^{1 / 8}\right)^{2} d_{2}^{2} d_{3} m$ for every $j=3, \ldots, s$;

(ii) the $(s-2,2)$-cylinder $\mathcal{H}(u, v)[\mathcal{H}(u)(v)]$ is $\left(2 \varepsilon_{3}^{1 / 32}, d_{3}^{2}, r^{\prime}\right)$-regular with respect to $\mathcal{G}[\mathcal{H}(u)(v)]$, and

(iii) the restriction $\mathcal{H}[\mathcal{G}(u, v)]$ is $\left(4 \varepsilon_{3}^{1 / 16}, d_{3}, r^{\prime}\right)$-regular with respect to $\mathcal{G}[\mathcal{G}(u, v)]$.

We denote by $N_{2}(u)_{\mathcal{H} \text {-nice }}$ the set of all $\mathcal{H}$-nice neighbors.

Remark. Recall that $\mathcal{H}(u, v)=\mathcal{H}(u) \cap \mathcal{H}(v)$ stands for the joint link of $u$ and $v$, whereas $\mathcal{H}(u)(v)$ is the neighborhood of $v$ in the graph $\mathcal{H}(u)$. 
The following observation shows that almost all $\mathcal{G}$-good neighbors are $\mathcal{H}$-nice.

Observation 9.2. Let $u \in U_{1}$ be an $\mathcal{H}$-nice vertex. Then all but at most $4\left(\begin{array}{c}s-2 \\ 2\end{array}\right) \varepsilon_{3}^{1 / 32} d_{2} m \mathcal{G}$-good neighbors in $N_{2}(u)_{\mathcal{G} \text {-good }}$ are $\mathcal{H}$-nice neighbors, i.e.

$$
\left|N_{2}(u)_{\mathcal{H} \text {-nice }}\right| \geq\left|N_{2}(u)_{\mathcal{G}-\text { good }}\right|-4\left(\begin{array}{c}
s-2 \\
2
\end{array}\right) \varepsilon_{3}^{1 / 32} d_{2} m .
$$

This observation follows from Claims 7.3 and 7.2 applied to cylinders $\mathcal{G}_{2}^{\prime}=\mathcal{G}[\mathcal{G}(u)], \mathcal{G}_{3}^{\prime}=\mathcal{H}(u)$ and $\mathcal{H}^{\prime}=\mathcal{H}[\mathcal{G}(u)]$.

Later, we will also need the fact that for every $\mathcal{H}$-nice vertex $u$ we have control over the number of edges in $\mathcal{H}$ that are also triangles in the joint neighborhood $\mathcal{H}(u)\left(v, v^{\prime}\right)$ for almost all pairs of vertices $\left\{v, v^{\prime}\right\} \subset N_{\mathcal{G}, 2}(u)$. Recall that $\mathcal{H}(u)\left(v, v^{\prime}\right)$ stands for the joint neighborhood of $\left\{v, v^{\prime}\right\}$ in $\mathcal{H}(u)$.

Claim 9.3. Suppose that $s=5$. Then for every $\mathcal{H}$-nice vertex $u \in U_{1}$, the following is true: all but at most $20 \varepsilon_{3}^{1 / 256} d_{2}^{2} m^{2}$ pairs $\left\{v, v^{\prime}\right\} \subset N_{\mathcal{G}, 2}(u)$ satisfy

$$
\left|\mathcal{H} \cap \mathcal{K}_{3}\left(\mathcal{H}\left(u, v, v^{\prime}\right)\left[\mathcal{H}(u)\left(v, v^{\prime}\right)\right]\right)\right| \leq 2 d_{2}^{12} d_{3}^{16} m^{3} .
$$

The proof of this claim is given in Appendix A.

\section{Regularity of the Links of $(s, 4)$-Cylinders}

In this section, we derive the two basics properties of the links of an $(s, 4)$ cylinder $\mathcal{F}$ : the regularity of the link $\mathcal{F}(u)$ and the regularity of $\mathcal{F}(u)(v)$, where $v$ is a neighbor of $u$. First, we describe our setup.

Setup D. Let $0<\varepsilon_{2} \ll d_{2} \leq 1,0<\varepsilon_{3} \ll d_{3} \leq 1$, and $0<\varepsilon_{4} \ll d_{4} \leq 1$ be real numbers so that $\varepsilon_{2} \ll \varepsilon_{3} \ll \varepsilon_{4}$. Let $U=U_{1} \cup \ldots \cup U_{s}$ be a partition, where $\left|U_{1}\right|=\ldots=\left|U_{s}\right|=m, \mathcal{G}=(U, E(\mathcal{G}))$ be an $(s, 2)$-cylinder that is $\left(\varepsilon_{2}, d_{2}\right)$-regular, $\mathcal{H}=(U, E(\mathcal{H}))$ be an $(s, 3)$-cylinder which is $\left(\varepsilon_{3}, d_{3}, r\right)$ regular with respect to $\mathcal{G}$, and let $\mathcal{F}=(U, E(\mathcal{F}))$ be an $(s, 4)$-cylinder which is $\left(\varepsilon_{4}, d_{4}, r\right)$-regular with respect to $\mathcal{H}$.

The following claim shows that the link $\mathcal{F}(u)$ "inherits" regularity from $\mathcal{F}$. It can be viewed as an analogy to Claim 6.1.

Claim 10.1. The $(s-1,3)$-cylinder $\mathcal{F}(u)$ is $\left(2 \varepsilon_{4}^{1 / 2}, d_{4}, r\right)$-regular with respect to $\mathcal{H} \cap \mathcal{K}_{3}(\mathcal{H}(u))$ for all but at most $4\left(\begin{array}{c}s-1 \\ 3\end{array}\right) \varepsilon_{4}^{1 / 2} m \mathcal{H}$-nice vertices $u \in U_{1}$.

Proof. Without loss of generality, we may assume $s=4$ and $U=U_{1} \cup U_{2} \cup$ $U_{3} \cup U_{4}$. Let $u$ be any $\mathcal{H}$-nice vertex. By Definition 6.3 we know

(i) the link $\mathcal{H}(u)$ is $\left(2 \varepsilon_{3}^{1 / 2}, d_{3}, r\right)$-regular with respect to $\mathcal{G}[\mathcal{G}(u)]$, and

(ii) $\mathcal{H}[\mathcal{G}(u)]$ is $\left(2 \varepsilon_{3}^{1 / 4}, d_{3}, r /\left(\varepsilon_{3}^{1 / 2} d_{2}^{-3}\right)\right)$-regular with respect to $\mathcal{G}[\mathcal{G}(u)]$.

Moreover, since $u$ must be also a $\mathcal{G}$-good vertex, we have (cf. Definition 4.7)

(iii) $\operatorname{deg}_{j}(u)=\left(d_{2} \pm \varepsilon_{2}\right) m$ for $j=2,3,4$,

(iv) the $(3,2)$-cylinder $\mathcal{G}[\mathcal{G}(u)]$ is $\left(\varepsilon_{2}^{1 / 2}, d_{2}\right)$-regular. 
We apply the 2-graphs Lemma (Lemma 5.4 with $\ell=2$ ) with

- $(3,2)$-cylinder $\mathcal{G}_{1}$ replaced by $\mathcal{G}[\mathcal{G}(u)]$ which is $\left(\varepsilon_{2}^{1 / 2}, d_{2}\right)$-regular (cf. (iv));

- $(3,2)$-cylinder $\mathcal{G}_{2}$ replaced by $\mathcal{H}(u)$ which is $\left(2 \varepsilon_{3}^{1 / 2}, d_{3}, r\right)$-regular with respect to $\mathcal{G}[\mathcal{G}(u)]$ (cf. (i));

- $U_{i-1}$ replaced by $\mathcal{G}(u) \cap U_{i}$ for $i=2,3,4$;

and obtain

$$
\left|\mathcal{K}_{3}(\mathcal{H}(u))\right|=\left(1 \pm\left(2 \varepsilon_{3}^{1 / 2}\right)^{1 / 64}\right) d_{2}^{3} d_{3}^{3} \times\left(\left(d_{2} \pm \varepsilon_{2}\right) m\right)^{3} .
$$

This can be further simplified using $\varepsilon_{2} \ll d_{2}$ and $\varepsilon_{3} \ll d_{3}$ to

$$
(3 / 4) d_{2}^{6} d_{3}^{3} m^{3} \leq\left|\mathcal{K}_{3}(\mathcal{H}(u))\right| \leq(5 / 4) d_{2}^{6} d_{3}^{3} m^{3} .
$$

It follows from Corollary 4.6 applied with $s=3$ and $\varepsilon_{3} \ll d_{3}$ that $2 \varepsilon_{3}^{1 / 4}\left|\mathcal{K}_{3}(\mathcal{G}[\mathcal{G}(u)])\right| \leq 2 \varepsilon_{3}^{1 / 4} \times(5 / 4) d_{2}^{3}\left(d_{2}+\varepsilon_{2}\right)^{3} m^{3} \leq(3 / 4) d_{2}^{6} d_{3}^{3} m^{3}$. Hence we have

$$
\left|\mathcal{K}_{3}(\mathcal{H}(u))\right| \geq 2 \varepsilon_{3}^{1 / 4}\left|\mathcal{K}_{3}(\mathcal{G}[\mathcal{G}(u)])\right| .
$$

Applying the $\left(2 \varepsilon_{3}^{1 / 4}, d_{3}, r /\left(\varepsilon_{3}^{1 / 2} d_{2}^{-3}\right)\right)$-regularity of $\mathcal{H}$ with respect to $\mathcal{G}[\mathcal{G}(u)]$ (cf. (ii)) yields

$$
\left|\mathcal{H} \cap \mathcal{K}_{3}(\mathcal{H}(u))\right|=\left(d_{3} \pm 2 \varepsilon_{3}^{1 / 4}\right)\left|\mathcal{K}_{3}(\mathcal{H}(u))\right| .
$$

We combine this inequality with (10.1) to conclude that for every $\mathcal{H}$-nice vertex $u$ we have

$$
(1 / 2) d_{2}^{6} d_{3}^{4} m^{3} \leq\left|\mathcal{H} \cap \mathcal{K}_{3}(\mathcal{H}(u))\right| \leq 2 d_{2}^{6} d_{3}^{4} m^{3} .
$$

Suppose that there are $t=2 \varepsilon_{4}^{1 / 2} m \mathcal{H}$-nice vertices $x_{1}, \ldots, x_{t} \in U_{1}$ such that for every $\alpha \in[t]$ the link $\mathcal{F}\left(u_{\alpha}\right)$ is $\left(2 \varepsilon_{2}^{1 / 4}, d_{4}, r\right)$-irregular with respect to $\mathcal{H} \cap \mathcal{K}_{3}\left(\mathcal{H}\left(u_{\alpha}\right)\right.$ ) (cf. Definition 5.1). Moreover, assume that for every $u_{\alpha}$ the second part of inequality (5.1) does not hold, that is, there exist $(3,2)$-cylinders $\mathcal{B}_{j \alpha} \subset \mathcal{H}\left(u_{\alpha}\right), j \in[r]$, such that

$$
\left|\mathcal{H} \cap \mathcal{K}_{3}\left(\mathcal{H}\left(u_{\alpha}\right)\right) \cap \bigcup_{j=1}^{r} \mathcal{K}_{3}\left(\mathcal{B}_{j \alpha}\right)\right| \geq 2 \varepsilon_{4}^{1 / 2}\left|\mathcal{H} \cap \mathcal{K}_{3}\left(\mathcal{H}\left(u_{\alpha}\right)\right)\right|
$$

but

$$
\left|\mathcal{F}\left(u_{\alpha}\right) \cap \bigcup_{j=1}^{r} \mathcal{K}_{3}\left(\mathcal{B}_{j \alpha}\right)\right|>\left(d_{4}+2 \varepsilon_{4}^{1 / 2}\right)\left|\mathcal{H} \cap \mathcal{K}_{3}\left(\mathcal{H}\left(u_{\alpha}\right)\right) \cap \bigcup_{j=1}^{r} \mathcal{K}_{3}\left(\mathcal{B}_{j \alpha}\right)\right| .
$$


For every $j \in[r]$, define a $(4,3)$-cylinder $Q_{j}=\partial_{1} Q_{j} \cup \partial_{2} Q_{j} \cup \partial_{3} Q_{j} \cup \partial_{4} Q_{j}$ by

$$
\begin{gathered}
\partial_{1} Q_{j}=\partial_{1} \mathcal{H}, \\
\partial_{2} Q_{j}=\bigcup_{\alpha=1}^{t}\left\{u_{\alpha} v w: v w \in \mathcal{B}_{j \alpha} \cap K\left(U_{3}, U_{4}\right)\right\}, \\
\partial_{3} Q_{j}=\bigcup_{\alpha=1}^{t}\left\{u_{\alpha} v w: v w \in \mathcal{B}_{j \alpha} \cap K\left(U_{2}, U_{4}\right)\right\}, \\
\partial_{4} Q_{j}=\bigcup_{\alpha=1}^{t}\left\{u_{\alpha} v w: v w \in \mathcal{B}_{j \alpha} \cap K\left(U_{2}, U_{3}\right)\right\} .
\end{gathered}
$$

We will show using $\left(\delta_{4}, d_{4}, r\right)$-regularity of $\mathcal{F}$ that

$$
\left|\mathcal{F} \cap \bigcup_{j=1}^{r} \mathcal{K}_{4}\left(Q_{j}\right)\right| \leq\left(d_{4}+\varepsilon_{4}\right) \sum_{\alpha=1}^{t}\left|\mathcal{H} \cap \bigcup_{j=1}^{r} \mathcal{K}_{3}\left(\mathcal{B}_{j \alpha}\right)\right|
$$

and then we use assumption (10.3b) to obtain a contradiction to this inequality.

Observe that since $\mathcal{B}_{j \alpha} \subset \mathcal{H}\left(u_{\alpha}\right)$ for every $j \in[r]$, we have

$$
\sum_{\alpha=1}^{t}\left|\mathcal{H} \cap \mathcal{K}_{3}\left(\mathcal{H}\left(u_{\alpha}\right)\right) \cap \bigcup_{j=1}^{r} \mathcal{K}_{3}\left(\mathcal{B}_{j \alpha}\right)\right|=\sum_{\alpha=1}^{t}\left|\mathcal{H} \cap \bigcup_{j=1}^{r} \mathcal{K}_{3}\left(\mathcal{B}_{j \alpha}\right)\right| .
$$

We estimate the size of $\bigcup_{j=1}^{r} \mathcal{K}_{4}\left(Q_{j}\right)$ as follows:

$$
\begin{aligned}
&\left|\bigcup_{j=1}^{r} \mathcal{K}_{4}\left(Q_{j}\right)\right|= \sum_{\alpha=1}^{t}\left|\mathcal{H} \cap \bigcup_{j=1}^{r} \mathcal{K}_{3}\left(\mathcal{B}_{j \alpha}\right)\right| \\
& \stackrel{(10.4)}{=} \sum_{\alpha=1}^{t}\left|\mathcal{H} \cap \mathcal{K}_{3}\left(\mathcal{H}\left(u_{\alpha}\right)\right) \cap \bigcup_{j=1}^{r}\right| \mathcal{K}_{3}\left(\mathcal{B}_{j \alpha}\right) \mid \\
& \stackrel{(10.3 \mathrm{a})}{\geq} \sum_{\alpha=1}^{t} 2 \varepsilon_{4}^{1 / 2}\left|\mathcal{H} \cap \mathcal{K}_{3}\left(\mathcal{H}\left(u_{\alpha}\right)\right)\right| \\
& \stackrel{(10.2)}{\geq} t \times 2 \varepsilon_{4}^{1 / 2} \times \frac{1}{2} d_{2}^{6} d_{3}^{4} m^{3} \\
&=2 \varepsilon_{4} d_{2}^{6} d_{3}^{4} m^{4} \geq \varepsilon_{4}\left|\mathcal{K}_{4}(\mathcal{H})\right| .
\end{aligned}
$$

The last inequality follows from the Theorem 1.13: $\mathcal{G}$ is a $\left(\delta_{2}, d_{2}\right)$-regular $(4,2)$-cylinder, $\mathcal{H}$ is a $(4,3)$-cylinder that is $\left(\delta_{3}, d_{3}, r\right)$-regular with respect to $\mathcal{G}$, and we can choose $\varepsilon_{2}$ and $\varepsilon_{3}$ so that the assumptions of Theorem 1.13 are satisfied. Thus, $\varepsilon_{4}\left|\mathcal{K}_{4}(\mathcal{H})\right| \leq \varepsilon_{4}(1 \pm \nu) d_{2}^{6} d_{3}^{4} m^{4} \leq 2 \varepsilon_{4} d_{2}^{6} d_{3}^{4} m^{4}$. 
Subsequently, the $\left(\varepsilon_{4}, d_{4}, r\right)$-regularity of $\mathcal{F}$ with respect to $\mathcal{H}$ implies that

$$
\begin{aligned}
\left|\mathcal{F} \cap \bigcup_{j=1}^{r} \mathcal{K}_{4}\left(Q_{j}\right)\right| & \leq\left(d_{4}+\varepsilon_{4}\right)\left|\bigcup_{j=1}^{r} \mathcal{K}_{4}\left(Q_{j}\right)\right| \\
& =\left(d_{4}+\varepsilon_{4}\right) \sum_{\alpha=1}^{t}\left|\mathcal{H} \cap \bigcup_{j=1}^{r} \mathcal{K}_{3}\left(\mathcal{B}_{j \alpha}\right)\right| .
\end{aligned}
$$

On the other hand, every $u_{\alpha}$ is contained in at most $\left|\mathcal{F}\left(u_{\alpha}\right) \cap \bigcup_{j=1}^{r} \mathcal{K}_{3}\left(\mathcal{B}_{j \alpha}\right)\right|$ triples (this follows from the definition of $Q_{j}$ ). We use $(10.3 \mathrm{~b})$ to conclude that

$$
\begin{aligned}
&\left|\mathcal{F} \cap \bigcup_{j=1}^{r} \mathcal{K}_{4}\left(Q_{j}\right)\right|=\sum_{\alpha=1}^{t}\left|\mathcal{F}\left(u_{\alpha}\right) \cap \bigcup_{j=1}^{r}\right| \mathcal{K}_{3}\left(\mathcal{B}_{j \alpha}\right) \mid \\
& \stackrel{(10.3 \mathrm{~b})}{>}\left(d_{4}+2 \varepsilon_{4}^{1 / 2}\right) \sum_{\alpha=1}^{t}\left|\mathcal{H} \cap \mathcal{K}_{3}\left(\mathcal{H}\left(u_{\alpha}\right)\right) \cap \bigcup_{j=1}^{r} \mathcal{K}_{3}\left(\mathcal{B}_{j \alpha}\right)\right|
\end{aligned}
$$

Comparing (10.6) with (10.5) we get a contradiction. Thus, there are at most $2 \varepsilon_{4}^{1 / 2} m$ vertices satisfying (10.3a) and (10.3b).

The case when the second part of inequality (5.1) is not true, i.e. when $(10.3 b)$ is replaced by

$$
\left|\mathcal{F}\left(u_{\alpha}\right) \cap \bigcup_{j=1}^{r} \mathcal{K}_{2}\left(\mathcal{B}_{j \alpha}\right)\right|<\left(d_{4}-2 \varepsilon_{4}^{1 / 2}\right)\left|\mathcal{H} \cap \mathcal{K}_{3}\left(\mathcal{H}\left(u_{\alpha}\right)\right) \cap \bigcup_{j=1}^{r} \mathcal{K}_{3}\left(\mathcal{B}_{j \alpha}\right)\right|
$$

is handled similarly.

The next claim shows that a majority of $\mathcal{H}$-nice vertices $u \in U_{1}$ have the property that the link $\mathcal{F}(u)(v)$ is regular for almost all $\mathcal{H}$-nice neighbors $v$ of $u$. We recall that $\mathcal{F}(u)(v)$ is an $(s-2,2)$-cylinder whose edges together with $u$ and $v$ form edges in $\mathcal{F}$.

Claim 10.2. For all but at most $2\left(\begin{array}{c}s-2 \\ 2\end{array}\right) \varepsilon_{4}^{1 / 4} m \mathcal{H}$-nice vertices $u \in U_{1}$ the following statement is true.

There are at most $2\left(\begin{array}{c}s-2 \\ 2\end{array}\right) \varepsilon_{4}^{1 / 4} d_{2} m \mathcal{H}$-nice neighbors $v \in N_{\mathcal{G}, 2}(u)$ for which the link $\mathcal{F}(u)(v)$ is not $\left(\varepsilon_{4}^{1 / 4}, d_{4}, r\right)$-regular with respect to $\mathcal{H}(u, v)[\mathcal{H}(u)(v)]$.

It is sufficient to consider the case $s=4$ only because we can treat the case $s>4$ by applying the result for $s=4$ to $\left(\begin{array}{c}s-2 \\ 2\end{array}\right)$ sets of cylinders induced on $U_{1} \cup U_{2} \cup U_{i} \cup U_{j}, 2<i<j \leq s$.

Proof. Set $r^{\prime}=r /\left(\varepsilon_{3}^{1 / 2} d_{2}^{-3}\right)\left(2 \varepsilon_{3}^{1 / 8} d_{2}^{-3}\right)$, let $u$ be an arbitrary $\mathcal{H}$-nice vertex, and $v$ be its $\mathcal{H}$-nice neighbor (cf. Definition 9.1). Then $v$ satisfies the following conditions:

(i) $\operatorname{deg}_{\mathcal{H}(u), j}(v)=\left(1 \pm 2 \varepsilon_{3}^{1 / 8}\right)^{3} d_{2}^{2} d_{3} m$ for $j=3,4$,

(ii) $\mathcal{G}[\mathcal{H}(u)(v)]$ is $\left(\varepsilon_{2}^{1 / 2}, d_{2}\right)$-regular, 
(iii) the link $\mathcal{H}(u, v)[\mathcal{H}(u)(v)]$ is $\left(2 \varepsilon_{3}^{1 / 32}, d_{3}^{2}, r^{\prime}\right)$-regular with respect to $\mathcal{G}[\mathcal{H}(u)(v)]$.

Observe that the $\left(2 \varepsilon_{3}^{1 / 32}, d_{3}^{2}, r^{\prime}\right)$-regularity of the link $\mathcal{H}(u, v)[\mathcal{H}(u)(v)]$ with respect to $\mathcal{G}[\mathcal{H}(u)(v)]$ is a sufficient condition to apply Observation 5.2. In a view of (i) and (ii), this observation implies

$$
(1 / 2) d_{2}^{5} d_{3}^{4} m^{2} \leq|\mathcal{H}(u, v)[\mathcal{H}(u)(v)]| \leq 2 d_{2}^{5} d_{3}^{4} m^{2} .
$$

Suppose there exist $t_{1}=\varepsilon_{4}^{1 / 4} m \mathcal{H}$-nice vertices $u_{1}, \ldots, u_{t_{1}} \in U_{1}$ so that for every $u_{\alpha}, \alpha \in\left[t_{1}\right]$, there are at least $t_{2}=\varepsilon_{4}^{1 / 4} d_{2} m \mathcal{H}$-nice neighbors $v_{1 \alpha}, \ldots, v_{t_{2} \alpha} \in N_{\mathcal{G}, 2}\left(u_{\alpha}\right)$ for which the link $\mathcal{F}\left(u_{\alpha}\right)\left(v_{\beta \alpha}\right), \beta \in\left[t_{2}\right]$, is not $\left(\varepsilon_{4}^{1 / 4}, d_{4}, r\right)$-regular with respect to $\mathcal{H}\left(u_{\alpha}, v_{\beta \alpha}\right)\left[\mathcal{H}\left(u_{\alpha}\right)\left(v_{\beta \alpha}\right)\right]$.

We further assume that the second part of inequality (5.1) is not satisfied, that is, for every $u_{\alpha}$ and $v_{\beta \alpha}$ there exist $(2,1)$-cylinders $\mathcal{B}_{j \beta \alpha}=Y_{j \beta \alpha} \cup W_{j \beta \alpha}$, $j \in[r]$, where $Y_{j \beta \alpha} \subset \mathcal{H}\left(u_{\alpha}\right)\left(v_{\beta \alpha}\right) \cap U_{3}$ and $W_{j \beta \alpha} \subset \mathcal{H}\left(u_{\alpha}\right)\left(v_{\beta \alpha}\right) \cap U_{4}$, such that

$$
\begin{aligned}
\left|\mathcal{H}\left(u_{\alpha}, v_{\beta \alpha}\right)\left[\mathcal{H}\left(u_{\alpha}\right)\left(v_{\beta \alpha}\right)\right] \cap \bigcup_{j=1}^{r} \mathcal{K}_{2}\left(\mathcal{B}_{j \beta \alpha}\right)\right| \\
\quad \geq \varepsilon_{4}^{1 / 4}\left|\mathcal{H}\left(u_{\alpha}, v_{\beta \alpha}\right)\left[\mathcal{H}\left(u_{\alpha}\right)\left(v_{\beta \alpha}\right)\right]\right|,
\end{aligned}
$$

but

$$
\begin{aligned}
\mid \mathcal{F}\left(u_{\alpha}\right)\left(v_{\beta \alpha}\right) & \cap \bigcup_{j=1}^{r} \mathcal{K}_{2}\left(\mathcal{B}_{j \beta \alpha}\right) \mid \\
& >\left(d_{4}+\varepsilon_{4}^{1 / 4}\right)\left|\mathcal{H}\left(u_{\alpha}, v_{\beta \alpha}\right)\left[\mathcal{H}\left(u_{\alpha}\right)\left(v_{\beta \alpha}\right)\right] \cap \bigcup_{j=1}^{r} \mathcal{K}_{2}\left(\mathcal{B}_{j \beta \alpha}\right)\right| .
\end{aligned}
$$

For every $j \in[r]$ define a $(4,3)$-cylinder $Q_{j}=\partial_{1} Q_{j} \cup \partial_{2} Q_{j} \cup \partial_{3} Q_{j} \cup \partial_{4} Q_{j}$ by

$$
\begin{gathered}
\partial_{1} Q_{j}=\partial_{1} \mathcal{H}, \quad \partial_{2} Q_{j}=\partial_{2} \mathcal{H} \\
\partial_{3} Q_{j}=\bigcup_{\alpha=1}^{t_{1}} \bigcup_{\beta=1}^{t_{2}}\left\{u_{\alpha} v_{\beta \alpha} z: z \in W_{j \beta \alpha}\right\}, \partial_{4} Q_{j}=\bigcup_{\alpha=1}^{t_{1}} \bigcup_{\beta=1}^{t_{2}}\left\{u_{\alpha} v_{\beta \alpha} z: z \in Y_{j \beta \alpha}\right\} .
\end{gathered}
$$

It follows from the above construction that

$$
\left|\bigcup_{j=1}^{r} \mathcal{K}_{4}\left(Q_{j}\right)\right|=\sum_{\alpha=1}^{t_{1}} \sum_{\beta=1}^{t_{2}}\left|\mathcal{H}\left(u_{\alpha}, v_{\beta \alpha}\right)\left[\mathcal{H}\left(u_{\alpha}\right)\left(v_{\beta \alpha}\right)\right] \cap \bigcup_{j=1}^{r} \mathcal{K}_{2}\left(\mathcal{B}_{j \beta \alpha}\right)\right|
$$


We use this equation together with the assumption (10.8a) and estimate (10.7) to conclude that $\left|\bigcup_{j=1}^{r} \mathcal{K}_{4}\left(Q_{j}\right)\right| \geq \varepsilon_{4}\left|\mathcal{K}_{4}(\mathcal{H})\right|$. Indeed,

$$
\begin{aligned}
& \sum_{\alpha=1}^{t_{1}} \sum_{\beta=1}^{t_{2}}\left|\mathcal{H}\left(u_{\alpha}, v_{\alpha}\right)\left[\mathcal{H}\left(u_{\alpha}\right)\left(v_{\beta \alpha}\right)\right] \cap \bigcup_{j=1}^{r} \mathcal{K}_{2}\left(\mathcal{B}_{j \beta \alpha}\right)\right| \\
& \stackrel{(10.8 \mathrm{a})}{\geq} \sum_{\alpha=1}^{t_{1}} \sum_{\beta=1}^{t_{2}} \varepsilon_{4}^{1 / 4}\left|\mathcal{H}\left(u_{\alpha}, v_{\beta \alpha}\right)\left[\mathcal{H}\left(u_{\alpha}\right)\left(v_{\beta \alpha}\right)\right]\right| \\
& \stackrel{(10.7)}{\geq} t_{1} \times t_{2} \times \varepsilon_{4}^{1 / 4} \times \frac{1}{2} d_{2}^{5} d_{3}^{4} m^{2}=\frac{1}{2} \varepsilon_{4}^{3 / 4} d_{2}^{6} d_{3}^{4} m^{4}>2 \varepsilon_{4} d_{2}^{6} d_{3}^{4} m^{4} .
\end{aligned}
$$

In the paragraph before equation (10.5), we showed $\left|\mathcal{K}_{4}(\mathcal{H})\right| \leq 2 d_{2}^{6} d_{3}^{4} m^{4}$ and, therefore, $\varepsilon_{4}\left|\mathcal{K}_{4}(\mathcal{H})\right| \leq \varepsilon_{4} 2 d_{2}^{6} d_{3}^{4} m^{4} \leq\left|\bigcup_{j=1}^{r} \mathcal{K}_{4}\left(Q_{j}\right)\right|$. Hence we can apply the $\left(\varepsilon_{4}, d_{4}, r\right)$-regularity of $\mathcal{F}$ with respect to $\mathcal{H}$. Then,

$$
\begin{aligned}
& \left|\mathcal{F} \cap \bigcup_{j=1}^{r} \mathcal{K}_{4}\left(Q_{j}\right)\right| \leq\left(d_{4}+\varepsilon_{4}\right)\left|\bigcup_{j=1}^{r} \mathcal{K}_{4}\left(Q_{j}\right)\right| \\
& \stackrel{(10.9)}{=}\left(d_{4}+\varepsilon_{4}\right) \sum_{\alpha=1}^{t_{1}} \sum_{\beta=1}^{t_{2}}\left|\mathcal{H}\left(u_{\alpha}, v_{\beta \alpha}\right)\left[\mathcal{H}\left(u_{\alpha}\right)\left(v_{\beta \alpha}\right)\right] \cap \bigcup_{j=1}^{r} \mathcal{K}_{2}\left(\mathcal{B}_{j \beta \alpha}\right)\right| .
\end{aligned}
$$

On the other hand, assumption (10.8b) yields

$$
\begin{aligned}
\mid \mathcal{F} & \cap \bigcup_{j=1}^{r} \mathcal{K}_{4}\left(Q_{j}\right)\left|=\sum_{\alpha=1}^{t_{1}} \sum_{\beta=1}^{t_{2}}\right| \mathcal{F}\left(u_{\alpha}\right)\left(v_{\beta \alpha}\right) \cap \bigcup_{j=1}^{r} \mathcal{K}_{2}\left(\mathcal{B}_{j \beta \alpha}\right) \mid \\
& \stackrel{(10.8 \mathrm{~b})}{>}\left(d_{4}+\varepsilon_{4}^{1 / 4}\right) \sum_{\alpha=1}^{t_{1}} \sum_{\beta=1}^{t_{2}}\left|\mathcal{H}\left(u_{\alpha}, v_{\beta \alpha}\right)\left[\mathcal{H}\left(u_{\alpha}\right)\left(v_{\beta \alpha}\right)\right] \cap \bigcup_{j=1}^{r} \mathcal{K}_{2}\left(\mathcal{B}_{j \beta \alpha}\right)\right| .
\end{aligned}
$$

Comparing inequalities (10.10) and (10.11) we get a contradiction. Hence $t_{1}<\varepsilon_{4}^{1 / 4} m$. If we assume that the first part of inequality (5.1) is not satisfied, we obtain contradiction in exactly the same way. Thus, for all but at most $2 \varepsilon_{4}^{1 / 4} m \mathcal{H}$-nice vertices $u \in U_{1}$ there are at most $2 \varepsilon_{4}^{1 / 4} d_{2} m \mathcal{H}$-nice neighbors $v \in N_{2}(u)_{\mathcal{H}-\text { nice }}$ such that the link $\mathcal{F}(u)(v)$ is not $\left(\varepsilon_{4}^{1 / 4}, d_{4}, r\right)$-regular with respect to $\mathcal{H}(u, v)[\mathcal{H}(u)(v)]$.

Definition 10.3 (fine vertex). An $\mathcal{H}$-nice vertex $u \in U_{1}$ is called $\mathcal{F}$-fine if it satisfies the following conditions:

(i) $\mathcal{F}(u)$ is $\left(2 \varepsilon_{4}^{1 / 2}, d_{4}, r\right)$-regular with respect to $\mathcal{H} \cap \mathcal{K}_{3}([\mathcal{H}(u)])$, and

(ii) $\mathcal{F}(u)(v)$ is not $\left(\varepsilon_{4}^{1 / 4}, d_{4}, r\right)$-regular with respect to $\mathcal{H}(u, v)[\mathcal{H}(u)(v)]$ for at most $2\left(\begin{array}{c}s-2 \\ 2\end{array}\right) \varepsilon_{4}^{1 / 4} d_{2} m \mathcal{H}$-nice neighbors $v \in N_{2}(u)_{\mathcal{H} \text {-nice. }}$

We denote by $U_{\mathcal{F} \text {-fine }}$ the set of all $\mathcal{F}$-fine vertices in $U_{1}$. 
Definition 10.4 (fine neighbor). Let $u \in U_{1}$ be an $\mathcal{F}$-fine vertex. A $\mathcal{H}$ nice neighbor $v \in N_{\mathcal{G}, 2}(u)$ is called $\mathcal{F}$-fine if the link $\mathcal{F}(u)(v)$ is $\left(\varepsilon_{4}^{1 / 4}, d_{4}, r\right)$ regular with respect to $\mathcal{H}(u, v)[\mathcal{H}(u)(v)]$. We denote by $N_{2}(u)_{\mathcal{F}-\text { fine }}$ the set of all $\mathcal{F}$-fine neighbors in $N_{\mathcal{G}, 2}(u)$.

Observe that $U_{\mathcal{F}-\text { fine }} \subset U_{\mathcal{H} \text {-nice }} \subset U_{\mathcal{G} \text {-good }} \subset U_{1}$ and $N_{2}(u)_{\mathcal{F}-\text { fine }} \subset$ $N_{2}(u)_{\mathcal{H} \text {-nice }} \subset N_{2}(u)_{\mathcal{G} \text {-good }} \subset N_{\mathcal{G}, 2}(u)$ for every (fine) vertex $u \in U_{1}$. Moreover, the following two observations are an easy consequence of Claims 10.2 and 10.1 .

Observation 10.5. All but at most $4\left(\begin{array}{c}s-1 \\ 3\end{array}\right) \varepsilon_{4}^{1 / 2} m+2\left(\begin{array}{c}s-2 \\ 2\end{array}\right) \varepsilon_{4}^{1 / 4} m \leq 6\left(\begin{array}{c}s-2 \\ 2\end{array}\right) \varepsilon_{4}^{1 / 4} m$ $\mathcal{H}$-nice vertices are $\mathcal{F}$-fine, that is

$$
\left|U_{\mathcal{F}-\text { fine }}\right| \geq\left|U_{\mathcal{H} \text {-nice }}\right|-6\left(\begin{array}{c}
s-2 \\
2
\end{array}\right) \varepsilon_{4}^{1 / 4} m
$$

Observation 10.6. Let $u \in U_{1}$ be an $\mathcal{F}$-fine vertex. Then all but at most $2\left(\begin{array}{c}s-2 \\ 2\end{array}\right) \varepsilon_{4}^{1 / 4} d_{2} m \mathcal{H}$-nice neighbors of $u$ are $\mathcal{F}$-fine, that is

$$
\left|N_{2}(u)_{\mathcal{F}-\text { fine }}\right| \geq\left|N_{2}(u)_{\mathcal{H} \text {-nice }}\right|-2\left(\begin{array}{c}
s-2 \\
2
\end{array}\right) \varepsilon_{4}^{1 / 4} d_{2} m .
$$

\section{Proof of Proposition 2.5}

Recall that our goal is to show that the number of copies of $K_{4}^{(3)}$ in $\mathcal{H}^{(4)}(x)$ satisfies

$$
\left|\mathcal{K}_{4}\left(\mathcal{H}^{(4)}(x)\right)\right|=(1 \pm \nu / 2) d_{4}^{4} d_{3}^{\left(\begin{array}{c}
5 \\
3
\end{array}\right)} d_{2}^{\left(\begin{array}{c}
5 \\
2
\end{array}\right)} n^{4} .
$$

for almost all vertices $x \in V_{1}$.

We are going to show that (11.12) is true for every $\mathcal{H}^{(4)}$-fine vertex $x \in V_{1}$ (cf. Definition 10.3 applied with $\mathcal{G}=\mathcal{H}^{(2)}, \mathcal{H}=\mathcal{H}^{(3)}, \mathcal{F}=\mathcal{H}^{(4)}, m=n$, $\varepsilon_{2}=\delta_{2}, \varepsilon_{3}=\delta_{3}$, and $\varepsilon_{4}=\delta_{4}$ ). It follows from Observation 10.5 (applied with $s=5$ ) that all but at most $18 \delta_{4}^{1 / 4} n \mathcal{H}^{(3)}$-nice vertices are $\mathcal{H}^{(4)}$-fine. Moreover, Observation 6.4 gives that all but at most $24 \delta_{3}^{1 / 2} n \mathcal{H}^{(2)}$-good vertices are $\mathcal{H}^{(3)}$-nice. Finally, from Observation 4.8 we have that all but at most $48 \delta_{2}^{1 / 4} n$ vertices in $V_{1}$ are $\mathcal{H}^{(2)}$-good. Altogether we obtain that all but at most $100 \delta_{4}^{1 / 4} n$ vertices $x \in V_{1}$ are $\mathcal{H}^{(4)}$-fine and, therefore, (11.12) holds.

Let $x \in V_{1}$ be a fixed $\mathcal{H}^{(4)}$-fine vertex. We divide the proof of (11.12) into five statements. Note that combining statements D and F yields (11.12).

A: (a) $\left|\mathcal{K}_{3}\left(\mathcal{H}^{(4)}(x)(y)\right)\right|=(1 \pm \nu / 6) d_{2}^{9} d_{3}^{9} d_{4}^{3} n^{3}$ for every vertex $y \in$ $N_{2}(x)_{\mathcal{H}^{(4)}-\text { fine }}$, and

(b) $(1 / 2) d_{2}^{9} d_{3}^{9} n^{3} \leq\left|\mathcal{K}_{3}\left(\mathcal{H}^{(3)}(x, y)\left[\mathcal{H}^{(3)}(x)(y)\right]\right)\right| \leq 2 d_{2}^{9} d_{3}^{9} n^{3}$ for every vertex $y \in N_{2}(x)_{\mathcal{H}^{(3)} \text {-nice. }}$.

B: (a) $\left|\mathcal{H}^{(3)} \cap \mathcal{K}_{3}\left(\mathcal{H}^{(4)}(x)(y)\right)\right|=(1 \pm \nu / 5) d_{2}^{9} d_{3}^{10} d_{4}^{3} n^{3}$ for every vertex $y \in N_{2}(x)_{\mathcal{H}^{(4)}}{ }_{\text {-fine }}$, and 
(b) $\left|\mathcal{H}^{(3)} \cap \mathcal{K}_{3}\left(\mathcal{H}^{(3)}(x, y) \mathcal{H}^{(3)}(x, y)\right)\right| \leq 3 d_{2}^{9} d_{3}^{10} n^{3}$ for every vertex $y \in N_{2}(x)_{\mathcal{H}^{(3)}-\text { nice }}$.

C: $\quad$ For every subset $W$ of $N_{2}(x)_{\mathcal{H}^{(4)} \text {-fine }}$ such that $|W| \geq 2 \delta_{4}^{1 / 4} d_{2} n$, there exist $t=\delta_{4}^{1 / 4} /\left(d_{2}^{3} d_{3}^{6}\right) \mathcal{H}^{(4)}$-fine neighbors $y_{1}, \ldots, y_{t} \in W$ of $x$ such that

$\left|\mathcal{H}^{(4)}(x) \cap \bigcup_{j=1}^{t} \mathcal{K}_{3}\left(\mathcal{H}^{(4)}(x)\left(y_{j}\right)\right)\right|=\left(d_{4} \pm 2 \delta_{4}^{1 / 2}\right)\left|\mathcal{H}^{(3)} \cap \bigcup_{j=1}^{t} \mathcal{K}_{3}\left(\mathcal{H}^{(4)}(x)\left(y_{j}\right)\right)\right|$.

D: $\quad\left|\mathcal{K}_{4}\left(\mathcal{H}^{(4)}(x)\right)\right| \geq(1-\nu / 2) d_{4}^{4} d_{3}^{\left(\begin{array}{l}5 \\ 3\end{array}\right)} d_{2}^{\left(\begin{array}{c}5 \\ 2\end{array}\right)} n^{4}$.

E: $\quad\left|\mathcal{K}_{4}\left(\mathcal{H}^{(4)}(x)\right)\right| \leq(1+\nu / 2) d_{4}^{4} d_{3}^{\left(\begin{array}{c}5 \\ 3\end{array}\right)} d_{2}^{\left(\begin{array}{c}5 \\ 2\end{array}\right)} n^{4}$.

Now we show statements A-E.

For the proofs of A and B, let $r^{\prime}=r /\left(\delta_{3}^{1 / 2} d_{2}^{-3}\right)\left(2 \delta_{3}^{1 / 8} d_{2}^{-3}\right)$. Recall that an arbitrary $\mathcal{H}^{(4)}$-fine neighbor $y$ of $x$ satisfies the following conditions (cf. Definition 10.4)

$\mathrm{A}(\mathrm{i})$ the link $\mathcal{H}^{(4)}(x)(y)$ is $\left(\delta_{4}^{1 / 4}, d_{4}, r\right)$-regular with respect to $\mathcal{H}^{(3)}(x, y)\left[\mathcal{H}^{(3)}(x)(y)\right]$. Since $y$ is also a $\mathcal{H}^{(3)}$-nice neighbor of $x$, it satisfies (cf. Definition 9.1)

$\mathrm{A}(\mathrm{ii}) \operatorname{deg}_{\mathcal{H}^{(3)}(x), j}(y)=\left(1 \pm 2 \delta_{3}^{1 / 8}\right)^{2} d_{2}^{2} d_{3} n$ for every $j=3,4,5$;

$\mathrm{A}(\mathrm{iii}) \mathcal{H}^{(3)}(x, y)\left[\mathcal{H}^{(3)}(x)(y)\right]$ is $\left(2 \delta_{3}^{1 / 32}, d_{3}^{2}, r^{\prime}\right)$-regular with respect to $\mathcal{H}^{(2)}\left[\mathcal{H}^{(3)}(x)(y)\right]$, and

$\mathrm{A}(\mathrm{iv}) \mathcal{H}^{(3)}\left[\mathcal{H}^{(2)}(x, y)\right]$ is $\left(4 \delta_{3}^{1 / 16}, d_{3}, r^{\prime}\right)$-regular with respect to $\mathcal{H}^{(2)}\left[\mathcal{H}^{(2)}(x, y)\right]$.

Moreover, $y$ is also an $\mathcal{H}^{(2)}$-good neighbor of $x$, thus we have (cf. Definition 4.9)

$\mathrm{A}(\mathrm{v})$ the link $\mathcal{H}^{(2)}\left[\mathcal{H}^{(2)}(x, y)\right]$ is $\left(\delta_{2}^{1 / 2}, d_{2}\right)$-regular, and

$\mathrm{A}(\mathrm{vi}) \operatorname{deg}_{j}(x, y)=\left(d_{2} \pm \delta_{2}^{1 / 2}\right)^{2} n$ holds for $j=3,4,5$.

Proof of A(a): Fix any $\mathcal{H}^{(4)}$-fine neighbor $y$ of $x$, then $\mathrm{A}(\mathrm{i})$-A(vi) hold.

We set

- $\mathcal{G}_{1}^{\prime}=\mathcal{H}^{(2)}\left[\mathcal{H}^{(3)}(x)(y)\right], \varepsilon_{1}^{\prime}=\delta_{2}^{1 / 2}, d_{1}^{\prime}=d_{2}$,

- $\mathcal{G}_{2}^{\prime}=\mathcal{H}^{(3)}(x, y)\left[\mathcal{H}^{(3)}(x)(y)\right], \varepsilon_{1}^{\prime}=2 \delta_{3}^{1 / 32}, d_{2}^{\prime}=d_{3}^{2}$,

- $\mathcal{G}_{3}^{\prime}=\mathcal{H}^{(4)}(x)(y), \varepsilon_{3}^{\prime}=\delta_{4}^{1 / 4}, d_{3}^{\prime}=d_{4}$, and

- $V_{i}^{\prime}=V_{i+2} \cap \mathcal{H}^{(3)}(x)(y), i=1,2,3$, and

- $m^{\prime}=\left(1 \pm 2 \delta_{3}^{1 / 8}\right)^{2} d_{2}^{2} d_{3} n$.

Observe that due to $\mathrm{A}(\mathrm{i}) \mathrm{A}(\mathrm{v}) \mathcal{G}_{1}^{\prime}, \mathcal{G}_{2}^{\prime}$, and $\mathcal{G}_{3}^{\prime}$ are (3,2)-cylinders which satisfy Setup A. Thus, we can apply the 3 -graphs Lemma and obtain that

$$
\left|\mathcal{K}_{3}\left(\mathcal{G}_{3}^{\prime}\right)\right|=\left(1 \pm \varepsilon_{3}^{\prime 1 / 64}\right)^{3}\left(d_{1}^{\prime} d_{2}^{\prime} d_{3}^{\prime}\right)^{3}\left(m^{\prime}\right)^{3} .
$$

We use the definition of $d_{1}^{\prime}, d_{2}^{\prime}, d_{3}^{\prime}, \varepsilon_{3}^{\prime}, m^{\prime}$, and $\mathcal{G}_{3}^{\prime}$, and the fact that $\delta_{3} \ll$ $\delta_{4} \ll \nu$ to conclude that

$$
\left|\mathcal{K}_{3}\left(\mathcal{H}^{(4)}(x)(y)\right)\right|=(1 \pm \nu / 6) d_{2}^{9} d_{3}^{9} d_{4}^{3} n^{3} .
$$


Proof of A(b): Fix any $\mathcal{H}^{(3)}$-nice neighbor $y$ of $x$. Then $y$ satisfies conditions $\mathrm{A}(\mathrm{ii})-\mathrm{A}(\mathrm{vi})$. We set

- $\mathcal{G}_{1}^{\prime}=\mathcal{H}^{(2)}\left[\mathcal{H}^{(3)}(x)(y)\right], \varepsilon_{1}^{\prime}=\delta_{2}^{1 / 2}, d_{1}^{\prime}=d_{2}$,

- $\mathcal{G}_{2}^{\prime}=\mathcal{H}^{(3)}(x, y)\left[\mathcal{H}^{(3)}(x)(y)\right], \varepsilon_{2}^{\prime}=2 \delta_{3}^{1 / 32}, d_{2}^{\prime}=d_{3}^{2}$.

Conditions $\mathrm{A}(\mathrm{ii})-\mathrm{A}(\mathrm{v})$ guarantee that $\mathcal{G}_{1}^{\prime}$ and $\mathcal{G}_{2}^{\prime}$ are $(3,2)$-cylinders satisfying Setup A. We apply the 2-graphs Lemma on $\mathcal{G}_{1}^{\prime}$ and $\mathcal{G}_{2}^{\prime}$ and obtain

$$
\left|\mathcal{K}_{3}\left(\mathcal{G}_{2}^{\prime}\right)\right|=\left(1 \pm \varepsilon_{2}^{\prime 1 / 64}\right)^{2}\left(d_{1}^{\prime} d_{2}^{\prime}\right)^{3}\left(m^{\prime}\right)^{3} .
$$

We use the definition of $d_{1}^{\prime}, d_{2}^{\prime}, \varepsilon_{2}^{\prime}, m^{\prime}$, and $\mathcal{G}_{2}^{\prime}$ together with the fact that $\delta_{3} \ll \delta_{4} \ll \nu$ to get

$$
\frac{1}{2} d_{2}^{9} d_{3}^{9} n^{3} \leq\left|\mathcal{K}_{3}\left(\mathcal{H}^{(3)}(x, y)\left[\mathcal{H}^{(3)}(x)(y)\right]\right)\right| \leq 2 d_{2}^{9} d_{3}^{9} n^{3} .
$$

Proof of B(a): Fix any $\mathcal{H}^{(4)}$-fine neighbor $y$ of $x$. Then $y$ satisfies conditions $\mathrm{A}(\mathrm{i})-\mathrm{A}(\mathrm{vi})$. For the proof of this part, we use the estimate from Part $\mathrm{A}(\mathrm{a})$ and the $\left(4 \delta_{3}^{1 / 16}, d_{3}, r^{\prime}\right)$-regularity of $\mathcal{H}^{(3)}\left[\mathcal{H}^{(2)}(x, y)\right]$ with respect to $\mathcal{H}^{(2)}\left[\mathcal{H}^{(2)}(x, y)\right]$ (cf. A(iv)). It follows from part $\mathrm{A}(\mathrm{a})$ that

$$
\left|\mathcal{K}_{3}\left(\mathcal{H}^{(4)}(x)(y)\right)\right|=(1 \pm \nu / 6) d_{2}^{9} d_{3}^{9} d_{4}^{3} n^{3} .
$$

Since $y$ satisfies $\mathrm{A}(\mathrm{v})$ and $\mathrm{A}(\mathrm{vi})$, we know that $\mathcal{H}^{(2)}\left[\mathcal{H}^{(2)}(x, y)\right]$ is $\left(\delta_{2}^{1 / 2}, d_{2}\right)$ regular and $\operatorname{deg}_{j}(x, y)=\left(d_{2} \pm \delta_{2}^{1 / 2}\right)^{2} n$ holds for $j=3,4,5$. We apply Corollary 4.6 and obtain $\left|\mathcal{K}_{3}\left(\mathcal{H}^{(2)}\left[\mathcal{H}^{(2)}(x, y)\right]\right)\right| \leq(5 / 4) d_{2}^{3}\left(d_{2}+\delta_{2}^{1 / 2}\right)^{6} n^{3} \leq$ $(3 / 2) d_{2}^{9} n^{3}$ (we used $\delta_{2} \ll d_{2}$ ). Furthermore, since $\delta_{3} \ll d_{3}, d_{4}, \nu$, we have

$$
\begin{aligned}
4 \delta_{3}^{1 / 16}\left|\mathcal{K}_{3}\left(\mathcal{H}^{(2)}\left[\mathcal{H}^{(2)}(x, y)\right]\right)\right| \leq & 6 \delta_{3}^{1 / 16} d_{2}^{9} n^{3} \\
& \leq(1-\nu / 6) d_{2}^{9} d_{3}^{9} d_{4}^{3} n^{3} \leq\left|\mathcal{K}_{3}\left(\mathcal{H}^{(4)}(x)(y)\right)\right| .
\end{aligned}
$$

Then we use the $\left(4 \delta_{3}^{1 / 16}, d_{3}, r^{\prime}\right)$-regularity of $\mathcal{H}^{(3)}\left[\mathcal{H}^{(2)}(x, y)\right]$ with respect to $\mathcal{H}^{(2)}\left[\mathcal{H}^{(2)}(x, y)\right]$ (cf. A(iv)), and obtain

$$
\left|\mathcal{H}^{(3)} \cap \mathcal{K}_{3}\left(\mathcal{H}^{(4)}(x)(y)\right)\right|=\left(d_{3} \pm 4 \delta_{3}^{1 / 16}\right)\left|\mathcal{K}_{3}\left(\mathcal{H}^{(4)}(x)(y)\right)\right| .
$$

We combine (11.13), (11.14), and assumption $\delta_{3} \ll d_{3} \ll \nu$ to get

$$
\left|\mathcal{H}^{(3)} \cap \mathcal{K}_{3}\left(\mathcal{H}^{(4)}(x)(y)\right)\right|=(1 \pm \nu / 5) d_{2}^{9} d_{3}^{10} d_{4}^{3} n^{3} .
$$

Proof B(b): Now $y$ is an $\mathcal{H}^{(3)}$-nice neighbor of an $\mathcal{H}^{(4)}$-fine vertex $x$, that is, a vertex satisfying $\mathrm{A}(\mathrm{ii})-\mathrm{A}(\mathrm{vi})$. Then, from Part $\mathrm{A}(\mathrm{b})$, we have

$$
\frac{1}{2} d_{2}^{9} d_{3}^{9} n^{3} \leq\left|\mathcal{K}_{3}\left(\mathcal{H}^{(3)}(x, y)\left[\mathcal{H}^{(3)}(x)(y)\right]\right)\right| \leq 2 d_{2}^{9} d_{3}^{9} n^{3} .
$$

Similarly to Part B(a), since $\delta_{3} \ll d_{3}$, we have

$$
\begin{aligned}
4 \delta_{3}^{1 / 16}\left|\mathcal{K}_{3}\left(\mathcal{H}^{(2)}\left[\mathcal{H}^{(2)}(x, y)\right]\right)\right| \leq & 6 \delta_{3}^{1 / 16} d_{2}^{9} n^{3} \\
& \leq \frac{1}{2} d_{2}^{9} d_{3}^{9} n^{3} \leq\left|\mathcal{K}_{3}\left(\mathcal{H}^{(3)}(x, y)\left[\mathcal{H}^{(3)}(x)(y)\right]\right)\right|
\end{aligned}
$$


We apply the $\left(4 \delta_{3}^{1 / 16}, d_{3}, r^{\prime}\right)$-regularity of $\mathcal{H}^{(3)}\left[\mathcal{H}^{(2)}(x, y)\right]$ with respect to $\mathcal{H}^{(2)}\left[\mathcal{H}^{(2)}(x, y)\right]$ (cf. A(iv)) and obtain

$$
\begin{aligned}
&\left|\mathcal{H}^{(3)} \cap \mathcal{K}_{3}\left(\mathcal{H}^{(3)}(x, y)\left[\mathcal{H}^{(3)}(x)(y)\right]\right)\right| \\
& \quad \leq\left(d_{3}+4 \delta_{3}^{1 / 16}\right)\left|\mathcal{K}_{3}\left(\mathcal{H}^{(3)}(x, y)\left[\mathcal{H}^{(3)}(x)(y)\right]\right)\right| \stackrel{(11.16)}{\leq} 3 d_{2}^{9} d_{3}^{10} n^{3} .
\end{aligned}
$$

Proof of C: Let $W$ be a subset of $N_{2}(x)_{\mathcal{H}^{(4)} \text {-fine }}$ such that $|W| \geq 2 \delta_{4}^{1 / 4} d_{2} n$. We define two graphs $\mathcal{P}_{1}$ and $\mathcal{P}_{2}$, both with vertex set $N_{\mathcal{H}^{(2)}, 2}(x)=\mathcal{H}^{(2)}(x) \cap$ $V_{2}$ and edge sets defined by:

$$
\begin{aligned}
& E\left(\mathcal{P}_{1}\right)=\left\{y y^{\prime}:\left|\mathcal{K}_{3}\left(\mathcal{H}^{(2)}\left[\mathcal{H}^{(2)}\left(x, y, y^{\prime}\right)\right]\right)\right|>2 d_{2}^{12} n^{3}\right\}, \\
& E\left(\mathcal{P}_{2}\right)=\left\{y y^{\prime}:\left|\mathcal{H}^{(3)} \cap \mathcal{K}_{3}\left(\mathcal{H}^{(3)}\left(x, y, y^{\prime}\right)\left[\mathcal{H}^{(3)}(x)\left(y, y^{\prime}\right)\right]\right)\right|>2 d_{3}^{16} d_{2}^{12} n^{3}\right\} .
\end{aligned}
$$

Now we estimate the sizes of $E\left(\mathcal{P}_{1}\right)$ and $E\left(\mathcal{P}_{2}\right)$. We start with $E\left(\mathcal{P}_{1}\right)$.

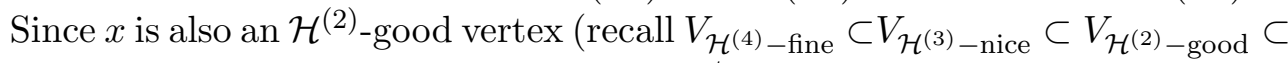
$\left.V_{1}\right)$, the $(4,2)$-cylinder $\mathcal{H}^{(2)}\left[\mathcal{H}^{(2)}(x)\right]$ is $\left(\delta_{2}^{1 / 2}, d_{2}\right)$-regular.

If $\left\{y, y^{\prime}\right\} \subset N_{\mathcal{H}^{(2)}, 2}(x)$ is $\mathcal{H}^{(2)}\left[\mathcal{H}^{(2)}(x)\right]$-good, then $\mathcal{H}^{(2)}\left[\mathcal{H}^{(2)}\left(x, y, y^{\prime}\right)\right]$ is $\left(\delta_{2}^{1 / 4}, d_{2}\right)$-regular and

$$
\operatorname{deg}_{j}\left(x, y, y^{\prime}\right)=\left(d_{2} \pm \delta_{2}^{1 / 4}\right)^{2}\left|N_{\mathcal{H}^{(2)}, j}(x)\right|
$$

for $j=3,4,5$ (cf. Definition 4.11(i) applied with $\mathcal{G}=\mathcal{H}^{(2)}\left[\mathcal{H}^{(2)}(x)\right]$ ). Since $x$ is $\mathcal{H}^{(2)}$-good, we have $\left|N_{\mathcal{H}^{(2)}, j}(x)\right|=\left(d_{2} \pm \delta_{2}\right) n$ and

$$
\operatorname{deg}_{j}\left(x, y, y^{\prime}\right)=\left(d_{2} \pm \delta_{2}^{1 / 4}\right)^{3} n,
$$

$j=3,4,5$.

Applying Corollary 4.6 with $\mathcal{G}=\mathcal{H}^{(2)}\left[\mathcal{H}^{(2)}\left(x, y, y^{\prime}\right)\right]$ yields

$$
\left|\mathcal{K}_{3}\left(\mathcal{H}^{(2)}\left[\mathcal{H}^{(2)}\left(x, y, y^{\prime}\right)\right]\right)\right| \leq(5 / 4) d_{2}^{3}\left(d_{2}+\delta_{2}^{1 / 4}\right)^{9} n^{3} \leq 2 d_{2}^{12} n^{3}
$$

(we used $\delta_{2} \ll d_{2}$ ).

Thus, $y y^{\prime} \notin E\left(\mathcal{P}_{1}\right)$ and $\left|E\left(\mathcal{P}_{1}\right)\right|$ is bounded by the number of pairs $\left\{y, y^{\prime}\right\}$ that are not $\mathcal{H}^{(2)}\left[\mathcal{H}^{(2)}(x)\right]$-good.

We apply Observations 4.8 and 4.12 on $\mathcal{G}=\mathcal{H}^{(2)}\left[\mathcal{H}^{(2)}(x)\right]$ and obtain that all but at most $66 \delta_{2}^{1 / 8}\left|N_{\mathcal{H}^{(2)}, 2}(x)\right|^{2}$ pairs $\left\{y, y^{\prime}\right\} \in N_{\mathcal{H}^{(2)}, 2}(x)$ are $\mathcal{H}^{(2)}\left[\mathcal{H}^{(2)}(x)\right]-$ good. Consequently,

$$
\left|E\left(\mathcal{P}_{1}\right)\right| \leq 66 \delta_{2}^{1 / 8}\left|N_{\mathcal{H}^{(2)}, 2}(x)\right|^{2} .
$$

Now we estimate the size of $E\left(\mathcal{P}_{2}\right)$. It follows from Claim 9.3 (used with $\mathcal{G}=\mathcal{H}^{(2)}$ and $\left.\mathcal{H}=\mathcal{H}^{(3)}\right)$ that for all but at most $20 \delta_{3}^{1 / 256} d_{2}^{2} n^{2}$ pairs $\left\{y, y^{\prime}\right\} \subset N_{\mathcal{H}^{(2), 2}}(x)$ we have

$$
\left|\mathcal{H}^{(3)} \cap \mathcal{K}_{3}\left(\mathcal{H}^{(3)}\left(x, y, y^{\prime}\right)\left[\mathcal{H}^{(3)}(x)\left(y, y^{\prime}\right)\right]\right)\right| \leq 2 d_{3}^{16} d_{2}^{12} n^{3} .
$$

Therefore,

$$
\left|E\left(\mathcal{P}_{2}\right)\right| \leq 20 \delta_{3}^{1 / 256} d_{2}^{2} n^{2} \leq 21 \delta_{3}^{1 / 256}\left|N_{\mathcal{H}^{(2)}, 2}(x)\right|^{2}
$$


We apply the Picking Lemma on $W$ with parameters $\sigma_{1}=66 \delta_{2}^{1 / 8}, \sigma_{2}=$ $21 \delta_{3}^{1 / 256}, t=\delta_{4}^{1 / 4} /\left(d_{2}^{3} d_{3}^{6}\right), c=\delta_{4}^{1 / 4}$, and obtain $t \mathcal{H}^{(4)}$-fine neighbors $y_{1}, \ldots$, $y_{t} \in W$ such that all pairs $\left\{y_{i}, y_{j}\right\}$ satisfy

$$
\left|\mathcal{K}_{3}\left(\mathcal{H}^{(2)}\left[\mathcal{H}^{(2)}\left(x, y_{i}, y_{j}\right)\right]\right)\right| \leq 2 d_{2}^{12} n^{3},
$$

and all but at most $\left(2 \times 2 \times 21 \delta_{3}^{1 / 256} / \delta_{4}^{1 / 2}\right) t^{2} \leq \delta_{3}^{1 / 512} t^{2}$ pairs $\left\{y_{i}, y_{j}\right\}$ satisfy

$$
\left|\mathcal{H}^{(3)} \cap \mathcal{K}_{3}\left(\mathcal{H}^{(3)}\left(x, y_{i}, y_{j}\right)\left[\mathcal{H}^{(3)}(x)\left(y_{i}, y_{j}\right)\right]\right)\right| \leq 2 d_{3}^{16} d_{2}^{12} n^{3} .
$$

This is possible as long as $|W| \geq 2 \delta_{4}^{1 / 4} d_{2} n \geq c \times\left|N_{\mathcal{H}^{(2)}, 2}(x)\right|$ and condition (2.8) is satisfied.

Now we estimate the size of $\mathcal{H}^{(3)} \cap \bigcup_{j=1}^{t} \mathcal{K}_{3}\left(\mathcal{H}^{(4)}(x)\left(y_{j}\right)\right)$. We first apply the Inclusion-Exclusion Principle:

$$
\begin{aligned}
\left|\bigcup_{j=1}^{t} \mathcal{H}^{(3)} \cap \mathcal{K}_{3}\left(\mathcal{H}^{(4)}(x)\left(y_{j}\right)\right)\right| \geq \sum_{j=1}^{t}\left|\mathcal{H}^{(3)} \cap \mathcal{K}_{3}\left(\mathcal{H}^{(4)}(x)\left(y_{j}\right)\right)\right| \\
-\sum_{1 \leq i<j \leq t}\left|\mathcal{H}^{(3)} \cap \mathcal{K}_{3}\left(\mathcal{H}^{(4)}(x)\left(y_{i}\right)\right) \cap \mathcal{K}_{3}\left(\mathcal{H}^{(4)}(x)\left(y_{j}\right)\right)\right| .
\end{aligned}
$$

The next step is to estimate both terms on the right-hand side. The first term is easier to handle. We use (11.15) to conclude that:

$$
\sum_{j=1}^{t}\left|\mathcal{H}^{(3)} \cap \mathcal{K}_{3}\left(\mathcal{H}^{(4)}(x)\left(y_{j}\right)\right)\right| \geq t \times(1-\nu / 5) d_{2}^{9} d_{3}^{10} d_{4}^{3} n^{3} .
$$

To get an estimate for the second term, we must observe two facts:

- $\mathcal{K}_{3}\left(\mathcal{H}^{(4)}(x)\left(y_{i}\right)\right) \cap \mathcal{K}_{3}\left(\mathcal{H}^{(4)}(x)\left(y_{j}\right)\right)=\mathcal{K}_{3}\left(\mathcal{H}^{(4)}(x)\left(y_{i}, y_{j}\right)\right)$, and

- $\mathcal{K}_{3}\left(\mathcal{H}^{(4)}(x)\left(y_{i}, y_{j}\right)\right) \subset \mathcal{K}_{3}\left(\mathcal{H}^{(3)}\left(x, y_{i}, y_{j}\right)\right) \subset \mathcal{K}_{3}\left(\mathcal{H}^{(2)}\left[\mathcal{H}^{(2)}\left(x, y_{i}, y_{j}\right)\right]\right)$.

Since we know that all but at most $\delta_{3}^{1 / 512} t^{2}$ pairs $\left\{y_{i}, y_{j}\right\}$ satisfy (11.18), for these pairs we use the estimate

$$
\begin{aligned}
\left|\mathcal{H}^{(3)} \cap \mathcal{K}_{3}\left(\mathcal{H}^{(4)}(x)\left(y_{i}, y_{j}\right)\right)\right| & \\
& \leq \mid \mathcal{H}^{(3)} \cap \mathcal{K}_{3}\left(\mathcal{H}^{(3)}\left(x, y_{i}, y_{j}\right)\left[\left(\mathcal{H}^{(3)}(x)\left(y_{i}, y_{j}\right)\right]\right) \mid\right. \\
& \stackrel{(11.18)}{\leq} 2 d_{3}^{16} d_{2}^{12} n^{3} .
\end{aligned}
$$

Remaining $\delta_{3}^{1 / 512} t^{2}$ pairs $\left\{y_{i}, y_{j}\right\}$ satisfy (11.17). For these pairs we use the following estimate

$$
\begin{aligned}
\left|\mathcal{H}^{(3)} \cap \mathcal{K}_{3}\left(\mathcal{H}^{(4)}(x)\left(y_{i}, y_{j}\right)\right)\right| & \\
\leq\left|\mathcal{K}_{3}\left(\mathcal{H}^{(2)}\left[\mathcal{H}^{(2)}\left(x, y_{i}, y_{j}\right)\right]\right)\right| & \stackrel{(11.17)}{\leq} 2 d_{2}^{12} n^{3} .
\end{aligned}
$$


Now we combine (11.20) and (11.21) to obtain

$$
\begin{aligned}
\sum_{1 \leq i<j \leq t} \mid \mathcal{H}^{(3)} \cap \mathcal{K}_{3}\left(\mathcal{H}^{(4)}(x)\left(y_{i}\right)\right. & \cap \mathcal{K}_{3}\left(\mathcal{H}^{(4)}(x)\left(y_{j}\right)\right) \mid \\
& \leq\left(\begin{array}{l}
t \\
2
\end{array}\right) \times 2 d_{3}^{16} d_{2}^{12} n^{3}+\delta_{3}^{1 / 512} t^{2} \times 2 d_{2}^{12} n^{3} .
\end{aligned}
$$

We use the assumption $\delta_{3} \ll d_{3}$ and conclude that $\delta_{3}^{1 / 512} t^{2} \times 2 d_{2}^{12} n^{3} \leq$ $t^{2} \times d_{3}^{16} d_{2}^{12} n^{3}$. Then,

$$
\sum_{1 \leq i<j \leq t}\left|\mathcal{H}^{(3)} \cap \mathcal{K}_{3}\left(\mathcal{H}^{(4)}(x)\left(y_{i}\right)\right) \cap \mathcal{K}_{3}\left(\mathcal{H}^{(4)}(x)\left(y_{j}\right)\right)\right| \leq 2 t^{2} d_{3}^{16} d_{2}^{12} n^{3} .
$$

Using (11.19), (11.22), and the definition of $t$ (recall $\left.t=\delta_{4}^{1 / 4} /\left(d_{2}^{3} d_{3}^{6}\right)\right)$ yields

$$
\begin{array}{r}
\left|\bigcup_{j=1}^{t} \mathcal{H}^{(3)} \cap \mathcal{K}_{3}\left(\mathcal{H}^{(4)}(x)\left(y_{j}\right)\right)\right| \geq t \times(1-\nu / 5) d_{2}^{9} d_{3}^{10} d_{4}^{3} n^{3}-2 t^{2} d_{3}^{16} d_{2}^{12} n^{3} \\
\stackrel{(2.3 \mathrm{a})}{\geq} 2 \delta_{4}^{1 / 2} \times 2 d_{3}^{4} d_{2}^{6} n^{3} .
\end{array}
$$

Since $x$ is an $\mathcal{H}^{(4)}$-fine vertex, the link $\mathcal{H}^{(4)}(x)$ is $\left(2 \delta_{4}^{1 / 2}, d_{4}, r\right)$-regular with respect to $\mathcal{H}^{(3)} \cap \mathcal{K}_{3}\left(\mathcal{H}^{(3)}(x)\right.$ ) (cf. Definition 10.3). Moreover, we know from $(10.2)$

$$
\frac{1}{2} d_{2}^{6} d_{3}^{4} n^{3} \leq\left|\mathcal{H}^{(3)} \cap \mathcal{K}_{3}\left(\mathcal{H}^{(3)}(x)\right)\right| \leq 2 d_{2}^{6} d_{3}^{4} n^{3} .
$$

We combine (11.23) and (11.24) and obtain

$$
\left|\bigcup_{j=1}^{t} \mathcal{H}^{(3)} \cap \mathcal{K}_{3}\left(\mathcal{H}^{(4)}(x)\left(y_{j}\right)\right)\right| \geq 2 \delta_{4}^{1 / 2}\left|\mathcal{H}^{(3)} \cap \mathcal{K}_{3}\left(\mathcal{H}^{(3)}(x)\right)\right| .
$$

The $\left(2 \delta_{4}^{1 / 2}, d_{4}, r\right)$-regularity of $\mathcal{H}^{(4)}(x)$ with respect to $\mathcal{H}^{(3)} \cap \mathcal{K}_{3}\left(\mathcal{H}^{(3)}(x)\right)$ yields (note that we can choose $r \geq t$ up-front (cf. (2.2))

$$
\left|\mathcal{H}^{(4)}(x) \cap \bigcup_{j=1}^{t} \mathcal{K}_{3}\left(\mathcal{H}^{(4)}(x)\left(y_{j}\right)\right)\right|=\left(d_{4} \pm 2 \delta_{4}^{1 / 2}\right)\left|\mathcal{H}^{(3)} \cap \bigcup_{j=1}^{t} \mathcal{K}_{3}\left(\mathcal{H}^{(4)}(x)\left(y_{j}\right)\right)\right|
$$

which is what we intended to show.

Proof of D: In Part C, we proved that whenever $W$ is a subset of $N_{2}(x)_{\mathcal{H}^{(4)} \text {-fine }}$ such that $|W| \geq 2 \delta_{4}^{1 / 4} d_{2} n$, we can choose $t=\delta_{4}^{1 / 4} /\left(d_{2}^{3} d_{3}^{6}\right) \mathcal{H}^{(4)}$-fine neighbors $y_{1}, \ldots, y_{t} \in W$ such that

$$
\left|\mathcal{H}^{(4)}(x) \cap \bigcup_{j=1}^{t} \mathcal{K}_{3}\left(\mathcal{H}^{(4)}(x)\left(y_{j}\right)\right)\right|=\left(d_{4} \pm 2 \delta_{4}^{1 / 2}\right)\left|\mathcal{H}^{(3)} \cap \bigcup_{j=1}^{t} \mathcal{K}_{3}\left(\mathcal{H}^{(4)}(x)\left(y_{j}\right)\right)\right|,
$$


Moreover, $y_{1}, \ldots, y_{t}$ also satisfy (11.19) and (11.22). Using these two equations and $t=\delta_{4}^{1 / 4} /\left(d_{2}^{3} d_{3}^{6}\right)$, we obtain

$$
\begin{gathered}
\delta_{4}^{1 / 8} \sum_{j=1}^{t}\left|\mathcal{H}^{(3)} \cap \mathcal{K}_{3}\left(\mathcal{H}^{(4)}(x)\left(y_{j}\right)\right)\right|-\sum_{1 \leq i<j \leq t}\left|\mathcal{H}^{(3)} \cap \mathcal{K}_{3}\left(\mathcal{H}^{(4)}(x)\left(y_{i}\right)\right) \cap \mathcal{K}_{3}\left(\mathcal{H}^{(4)}(x)\left(y_{j}\right)\right)\right| \\
\geq \delta_{4}^{1 / 8} \times t \times(1-\nu / 5) d_{2}^{9} d_{3}^{10} d_{4}^{3} n^{3}-2 t^{2} d_{3}^{16} d_{2}^{12} n^{3} \geq 0 .
\end{gathered}
$$

We apply the second part of Observation 2.7:

$$
\left|\bigcup_{j=1}^{t} \mathcal{H}^{(3)} \cap \mathcal{K}_{3}\left(\mathcal{H}^{(4)}(x)\left(y_{j}\right)\right)\right| \geq\left(1-\delta_{4}^{1 / 8}\right) \sum_{j=1}^{t}\left|\mathcal{H}^{(3)} \cap \mathcal{K}_{3}\left(\mathcal{H}^{(4)}(x)\left(y_{j}\right)\right)\right| .
$$

We combine (11.25), (11.26), and (11.19) and get

$$
\left|\mathcal{H}^{(4)}(x) \cap \bigcup_{j=1}^{t} \mathcal{K}_{3}\left(\mathcal{H}^{(4)}(x)\left(y_{j}\right)\right)\right| \geq\left(d_{4}-2 \delta_{4}^{1 / 2}\right)\left(1-\delta_{4}^{1 / 8}\right) t \times(1-\nu / 5) d_{2}^{9} d_{3}^{10} d_{4}^{3} n^{3} .
$$

We set $W=N_{2}(x)_{\mathcal{H}^{(4)}-\text { fine }}$ and find vertices $y_{1}, \ldots, y_{t}$ as described above. Then we remove $y_{1}, \ldots, y_{t}$ from $W$ and iterate the whole process again. We can repeat this process as long as (cf. Part C)

$$
|W| \geq 2 \delta_{4}^{1 / 4} d_{2} n \text {. }
$$

This way we produce a sequence of at least $\left(\left|N_{2}(x)_{\mathcal{H}^{(4)}-\text { fine }}\right|-2 \delta_{4}^{1 / 4} d_{2} n\right) / t$ $t$-tuples $Y^{(1)}=\left\{y_{1}, \ldots, y_{t}\right\}=\left\{y_{1}^{(1)}, \ldots, y_{t}^{(1)}\right\}, Y^{(2)}=\left\{y_{1}^{(2)}, \ldots, y_{t}^{(2)}\right\}$, etc. Analogously to (11.27), each iteration produces at least

$$
\left(d_{4}-2 \delta_{4}^{1 / 2}\right)\left(1-\delta_{4}^{1 / 8}\right) t \times(1-\nu / 5) d_{2}^{9} d_{3}^{10} d_{4}^{3} n^{3} \geq(1-\nu / 4) d_{2}^{9} d_{3}^{10} d_{4}^{4} t n^{3}
$$

copies of $K_{4}^{(3)}$ in $\mathcal{H}^{(4)}(x)$. Each such a copy uses exactly one vertex from $Y^{(i)}=\left\{y_{1}^{(i)}, \ldots, y_{t}^{(i)}\right\}$. Notice that since $x$ is an $\mathcal{H}^{(4)}$-fine vertex,

- $\left|N_{2}(x)_{\mathcal{H}^{(4)} \text {-fine }}\right| \geq\left|N_{2}(x)_{\mathcal{H}^{(3)}-\text { nice }}\right|-6 \delta_{4}^{1 / 4} d_{2} n$ (cf. Observation 10.6),

- $\left|N_{2}(x)_{\mathcal{H}^{(3)} \text {-nice }}\right| \geq\left|N_{2}(x)_{\mathcal{H}^{(2)} \text {-good }}\right|-12 \delta_{3}^{1 / 32} d_{2} n$ (cf. Observation 9.2),

- $\left|N_{2}(x)_{\mathcal{H}^{(2)} \text {-good }}\right| \geq\left|N_{\mathcal{H}^{(2)}, 2}(x)\right|-36 \delta_{2}^{1 / 8}\left|N_{\mathcal{H}^{(2)}, 2}(x)\right|$ (cf. Observation 4.10), and

- $\left|N_{\mathcal{H}^{(2)}, 2}(x)\right| \geq\left(d_{2}-\delta_{2}\right) n$ (cf. Definition 4.7).

Consequently, $\left(\left|N_{2}(x)_{\mathcal{H}^{(4)} \text {-fine }}\right|-2 \delta_{4}^{1 / 4} d_{2} n\right) / t \geq\left(1-9 \delta_{4}^{1 / 4}\right) d_{2} n / t$. Therefore, the sequence of $t$-tuples $X^{(i)}$ produces at least $\left(1-9 \delta_{4}^{1 / 4}\right) d_{2} n / t \times(1-$ $\nu / 4) d_{2}^{9} d_{3}^{10} d_{4}^{4} t n^{3}$ copies of $K_{4}^{(3)}$ in $\mathcal{H}^{(4)}(x)$. Hence

$$
\begin{aligned}
&\left|\mathcal{K}_{4}\left(\mathcal{H}^{(4)}(x)\right)\right| \geq\left(1-7 \delta_{4}^{1 / 4}\right) d_{2} n / t \times(1-\nu / 4) d_{2}^{9} d_{3}^{10} d_{4}^{4} t n^{3} \\
& \stackrel{(2.3 \mathrm{a})}{\geq}(1-\nu / 2) d_{2}^{\left(\begin{array}{l}
5 \\
3
\end{array}\right)} d_{3}^{\left(\begin{array}{l}
5 \\
2
\end{array}\right)} d_{4}^{4} n^{4} .
\end{aligned}
$$


Proof of E: Similarly to statement D, we set $W=N_{2}(x)_{\mathcal{H}^{(4)}}$-fine and iteratively produce $t$-tuples of vertices $Y^{(1)}, Y^{(2)}, \ldots, Y^{\text {(last) }}$, so that every $t$-tuple $Y^{(i)}$ is in at most $\left(d_{4}+2 \delta_{4}^{1 / 2}\right) \times t(1+\nu / 5) d_{4}^{3} d_{3}^{10} d_{2}^{9} n^{3}$ copies of $K_{4}^{(3)}$ in $\mathcal{H}^{(4)}(x)$.

However, to prove the upper bound we must count not only

(i) the contribution of $t$-tuples $Y^{(1)}, Y^{(2)}, \ldots, Y^{(\text {last })}$, but also

(ii) contribution of fine neighbors left in $N_{2}(x)_{\mathcal{H}^{(4)}-\text { fine }} \backslash \bigcup_{i=1}^{\text {last }} Y^{(i)}$, and

(iii) contribution of $y \notin N_{2}(x)_{\mathcal{H}^{(4)} \text {-fine }}$.

We will handle each of these categories of vertices separately:

(i) An upper bound on number of copies of $K_{4}^{(3)}$ produced by taking $t$-tuples $Y^{(i)}$ can be obtained in a way similar to the lower bound in Part D: There are at most $\left|N_{2}(x)_{\mathcal{H}^{(4)} \text {-fine }}\right| / t \leq\left|N_{\mathcal{H}^{(2)}, 2}(x)\right| / t \leq\left(d_{2}+\delta_{2}\right) n / t$ such $t$-tuples, together producing at most

$$
\left(d_{4}+2 \delta_{4}^{1 / 2}\right) \times t(1+\nu / 5) d_{4}^{3} d_{3}^{10} d_{2}^{9} n^{3} \times\left(d_{2}+\delta_{2}\right) n / t \leq(1+\nu / 4) d_{2}^{\left(\begin{array}{c}
5 \\
3
\end{array}\right)} d_{3}^{\left(\begin{array}{c}
5 \\
2
\end{array}\right)} d_{4}^{4} n^{4}
$$

copies of $K_{4}^{(3)}$.

(ii) The number of fine neighbors left in $N_{2}(x)_{\mathcal{H}^{(4)}-\text { fine }} \backslash \bigcup_{i=1}^{\text {last }} Y^{(i)}$ is at most $2 \delta_{4}^{1 / 4} d_{2} n$ (cf. (11.28)). Each such vertex satisfies $\mathrm{B}(\mathrm{a})$ and, consequently, is involved in not more than $(1+\nu / 5) d_{2}^{9} d_{3}^{10} d_{4}^{3} n^{3}$ copies of $K_{4}^{(3)}$. Therefore, this group of vertices contributes at most

$$
2 \delta_{4}^{1 / 4} d_{2} n \times(1+\nu / 5) d_{2}^{9} d_{3}^{10} d_{4}^{3} n^{3}<\delta_{4}^{1 / 8} d_{2}^{\left(\begin{array}{c}
5 \\
2
\end{array}\right)} d_{3}^{\left(\begin{array}{c}
5 \\
3
\end{array}\right)} d_{4}^{4} n^{4}
$$

copies of $K_{4}^{(3)}$. We used again the assumption $\delta_{4} \ll d_{4}$.

(iii) Now we must estimate the contribution of neighbors $y$ which are not $\mathcal{H}^{(4)}$-fine, that is, $y \in N_{\mathcal{H}^{(2)}, 2}(x) \backslash N_{2}(x)_{\mathcal{H}^{(4)} \text {-fine. }}$. Since

$$
\begin{aligned}
& N_{\mathcal{H}^{(2)}, 2}(x) \backslash N_{2}(x)_{\mathcal{H}^{(4)} \text {-fine }}=\left(N_{2}(x)_{\mathcal{H}^{(3)} \text {-nice }} \backslash N_{2}(x)_{\mathcal{H}^{(4)} \text {-fine }}\right) \cup \\
& \left(N_{2}(x)_{\mathcal{H}^{(2)} \text {-good }} \backslash N_{2}(x)_{\mathcal{H}^{(3)} \text {-nice }}\right) \cup\left(N_{\mathcal{H}^{(2)}, 2}(x) \backslash N_{2}(x)_{\mathcal{H}^{(2)} \text {-good }}\right),
\end{aligned}
$$

we distinguish three categories of these neighbors.

1) we consider vertices $y \in N_{2}(x)_{\mathcal{H}^{(3)} \text {-nice }} \backslash N_{2}(x)_{\mathcal{H}^{(4)} \text {-fine. }}$. We know from Observation 10.6 that $\left|N_{2}(x)_{\mathcal{H}^{(3)} \text {-nice }} \backslash N_{2}(x)_{\mathcal{H}^{(4)} \text {-fine }}\right| \leq 6 \delta_{4}^{1 / 4} d_{2} n$. We estimate contribution of every such vertex $y$ by

$$
\left|\mathcal{H}^{(3)} \cap \mathcal{K}_{3}\left(\mathcal{H}^{(4)}(x)(y)\right)\right| \leq\left|\mathcal{H}^{(3)} \cap \mathcal{K}_{3}\left(\mathcal{H}^{(3)}(x, y)\left[\mathcal{H}^{(3)}(x)(y)\right]\right)\right| \leq 3 d_{2}^{9} d_{3}^{10} n^{3}
$$

(cf. B(b)). Therefore, neighbors from $N_{2}(x)_{\mathcal{H}^{(3)} \text {-nice }} \backslash N_{2}(x)_{\mathcal{H}^{(4)} \text {-fine }}$ contribute by at most

$$
6 \delta_{4}^{1 / 4} d_{2} n \times 3 d_{3}^{10} d_{2}^{9} n^{3} \leq \delta_{4}^{1 / 8} d_{4}^{4} d_{3}^{\left(\begin{array}{c}
5 \\
3
\end{array}\right)} d_{2}^{\left(\begin{array}{c}
5 \\
2
\end{array}\right)} n^{4}
$$

copies of $K_{4}^{(3)}$. 
2) for vertices $y \in N_{2}(x)_{\mathcal{H}^{(2)} \text {-good }} \backslash N_{2}(x)_{\mathcal{H}^{(3)} \text {-nice, }}$, Observation $9.2 \mathrm{im}$ plies that $\left|N_{2}(x)_{\mathcal{H}^{(2)} \text {-good }} \backslash N_{2}(x)_{\mathcal{H}^{(3)} \text {-nice }}\right| \leq 12 \delta_{3}^{1 / 32} d_{2} n$. Then, each such neighbor $y$ is in at most $\left|\mathcal{K}_{3}\left(\mathcal{H}^{(2)}\left[\mathcal{H}^{(2)}(x, y)\right)\right]\right|$ copies of $K_{4}^{(3)}$. Since $y \in$ $\left.N_{2}(x)_{\mathcal{H}^{(2)} \text {-good }}, \mathcal{H}^{(2)}\left[\mathcal{H}^{(2)}(x, y)\right)\right]$ is $\left(\delta_{2}^{1 / 4}, d_{2}\right)$-regular (cf. Definition 9.1). Consequently,

$$
\left|\mathcal{K}_{3}\left(\mathcal{H}^{(2)}\left[\mathcal{H}^{(2)}(x, y)\right)\right]\right| \leq 2 d_{2}^{9} n^{3}
$$

(cf. Corollary 4.6). The total contribution of these vertices is then bounded by

$$
12 \delta_{3}^{1 / 32} d_{2} n \times 2 d_{2}^{9} n^{3} \leq \delta_{3}^{1 / 64} d_{3}^{\left(\begin{array}{c}
5 \\
3
\end{array}\right)} d_{2}^{\left(\begin{array}{c}
5 \\
2
\end{array}\right)} n^{4} \leq \delta_{4} d_{3}^{\left(\begin{array}{c}
5 \\
3
\end{array}\right)} d_{2}^{\left(\begin{array}{c}
5 \\
2
\end{array}\right)} n^{4} \leq \delta_{4}^{1 / 2} d_{4}^{4} d_{3}^{\left(\begin{array}{c}
5 \\
3
\end{array}\right)} d_{2}^{\left(\begin{array}{c}
5 \\
2
\end{array}\right)} n^{4} .
$$

Here we used assumptions (2.3a) and (2.3b).

3) The remaining neighbors $y$ belongs to $N_{\mathcal{H}^{(2), 2}}(x) \backslash N_{2}(x)_{\mathcal{H}^{(2)} \text {-good }}$. It follows from Observation 4.10 that $\left|N_{\mathcal{H}^{(2)}, 2}(x) \backslash N_{2}(x)_{\mathcal{H}^{(2)} \text {-good }}\right| \leq 36 \delta_{2}^{1 / 8}\left(d_{2}+\right.$ $\left.\delta_{2}\right) n \leq 37 \delta_{2}^{1 / 8} d_{2} n$. In this case, we use a rough estimate that every vertex is in at most $n^{3}$ copies of $K_{4}^{(3)}$ and, thus, the contribution of these vertices is at most

$$
\begin{aligned}
37 \delta_{2}^{1 / 8} d_{2} n \times n^{3} \leq & \delta_{2}^{1 / 16} d_{2}^{\left(\begin{array}{c}
5 \\
2
\end{array}\right)} n^{4} \leq \delta_{3} d_{2}^{\left(\begin{array}{c}
5 \\
2
\end{array}\right)} n^{4} \\
& \leq \delta_{3}^{1 / 2} d_{3}^{\left(\begin{array}{c}
5 \\
3
\end{array}\right)} d_{2}^{\left(\begin{array}{l}
5 \\
2
\end{array}\right)} n^{4} \leq \delta_{4} d_{3}^{\left(\begin{array}{c}
5 \\
3
\end{array}\right)} d_{2}^{\left(\begin{array}{l}
5 \\
2
\end{array}\right)} n^{4} \leq \delta_{4}^{1 / 2} d_{4}^{4} d_{3}^{\left(\begin{array}{l}
5 \\
3
\end{array}\right)} d_{2}^{\left(\begin{array}{c}
5 \\
2
\end{array}\right)} n^{4} .
\end{aligned}
$$

At this point we are ready to derive the upper bound. We add the contributions of all vertices above and obtain

$\left|\mathcal{K}_{4}\left(\mathcal{H}^{(4)}(x)\right)\right| \leq\left(1+\nu / 4+2 \delta_{4}^{1 / 8}+2 \delta_{4}^{1 / 2}\right) d_{4}^{4} d_{3}^{\left(\begin{array}{c}5 \\ 3\end{array}\right)} d_{2}^{\left(\begin{array}{c}5 \\ 2\end{array}\right)} n^{4} \leq(1+\nu / 2) d_{4}^{4} d_{3}^{\left(\begin{array}{c}5 \\ 3\end{array}\right)} d_{2}^{\left(\begin{array}{c}5 \\ 2\end{array}\right)} n^{4}$

\section{REFERENCES}

[Chu91] F.R.K. Chung, Regularity lemmas for hypergraphs and quasi-randomness, Random Structures Algorithms 2 (1991), no. 1, 241-252.

[CRST83] V. Chvátal, V. Rödl, E. Szemerédi, and W.T. Trotter, The Ramsey number of a graph with bounded maximum degree, J. Combin. Theory, Series B 34 (1983), no. 3, 239-243.

[DHNR02] Y. Dementieva, P. Haxell, B. Nagle, and V. Rödl, On characterizing triple system regularity, Random Structures Algorithms 21 (2002), no. 2-3, 293-335.

[FR92] P. Frankl and V. Rödl, The uniformity lemma for hypergraphs, Graphs Combin. 8 (1992), no. 4, 309-312.

[FR02] - Extremal problems on set systems, Random Structures Algorithms 20 (2002), no. 2, 131-164.

[KNR03] Y. Kohayakawa, B. Nagle, and V. Rödl, Hereditary properties of triple systems, Combin. Probab. Comput. 12 (2003), no. 2, 155-189.

[KSSS02] J. Komlós, A. Shokoufandeh, M. Simonovits, E. Szemerédi, The regularity lemma and its applications in graph theory, in Theoretical aspects of computer science (Tehran, 2000), 84-112, Lecture Notes in Comput. Sci., 2292, Springer, Berlin, 2002 
[KS96] J. Komlós and M. Simonovits, Szemerédi's regularity lemma and its applications in graph theory, Combinatorics - Paul Erdös is eighty, vol. 2 (Keszthely, 1993) (D. Miklós, V.T. Sós, and T. Szőnyi, eds.), Bolyai Society Mathematical Studies, vol. 2, János Bolyai Mathematical Society, Budapest, 1996, pp. 295352.

[KSS97] J. Komlós, G.N. Sárközy, and E. Szemerédi, Blow-up Lemma, Combinatorica 17 (1997), no. 1, 109-123.

[Ma02] J. Matoušek, Lectures on discrete geometry, Graduate Texts in Mathematics 212, Springer-Verlag, Berlin, 2002, xvi+481 pp.

[NR03] B. Nagle and V. Rödl, Regularity properties for triple systems, Random Structures Algorithms 23 (2003), no. 3, 264-332.

[NR02] - The asymptotic number of triple systems not containing a fixed one, Discrete Math. 235 (2002), no 1-3, 271-290.

[PRS04] Y. Peng, V. Rödl, and J. Skokan, Counting small cliques in 3-uniform hypergraphs, Combin. Probab. Comput. 14 (2004), 1-43.

[Ro91] V. Rödl, Some developments in Ramsey theory, Proceedings of the International Congress of Mathematicians, Vol. I, II (Kyoto, 1990), Math. Soc. Japan, Tokyo, 1991, 1455-1466.

[RR98] V. Rödl and A. Ruciński, Ramsey properties of random hypergraphs, J. Combin. Theory, Series A 81 (1998), no. 1, 1-33.

[RS04] V. Rödl and J. Skokan, Regularity lemma for $k$-uniform hypergraphs, Random Structures Algorithms 25 (2004), no. 1, 1-42.

[RS04a] Applications of the regularity lemma for uniform hypergraphs, submitted, 2004.

[RS04b] - Applications of the l-graphs Lemma, in preparation.

[Sk00] J. Skokan, Uniformity of Set Systems, PhD Thesis, Emory University, 2000 (available at http://www.mathcs.emory.edu/ rodl/grads.html).

[Sze75] E. Szemerédi, On sets of integers containing no $k$ elements in arithmetic progression, Acta Arithmetica 27 (1975), 199-245, collection of articles in memory of Juriǐ Vladimirovič Linnik.

[Sze78] _ Regular partitions of graphs, Problèmes Combinatoires et Théorie des Graphes (Colloq. Internat. CNRS, Univ. Orsay, Orsay, 1976)(Paris), Colloques Internationaux CNRS n. 260, 1978, pp. 399-401.

\section{Appendix A. Counting II}

In this section, we will expand statements for a single vertex from Section 8 to pairs. We consider the scenario given by Setup C:

Setup C. Let $0<\varepsilon_{2} \ll d_{2} \leq 1,0<\varepsilon_{3} \ll d_{3} \leq 1$, and $0<\varepsilon_{3} \ll \tilde{d}_{3} \leq 1$ be real numbers so that $\varepsilon_{2} \ll \varepsilon_{3}$. Let $U=U_{1} \cup \ldots \cup U_{s}$ be a partition, where $\left|U_{1}\right|=\ldots=\left|U_{s}\right|=m, \mathcal{G}_{2}=\left(U, E\left(\mathcal{G}_{2}\right)\right)$ be an $(s, 2)$-cylinder that is $\left(\varepsilon_{2}, d_{2}\right)$-regular, $\mathcal{G}_{3}=\left(U, E\left(\mathcal{G}_{3}\right)\right)$ be an $(s, 2)$-cylinder that is $\left(\varepsilon_{3}, \tilde{d}_{3}, r\right)$ regular with respect to $\mathcal{G}_{2}$, and let $\mathcal{H}=(U, E(\mathcal{H}))$ be an $(s, 3)$-cylinder which is $\left(\varepsilon_{3}, d_{3}, r\right)$-regular with respect to $\mathcal{G}_{2}$.

Our objective is to prove the following technical claim.

Claim A.1. Let $s=4$ and $\mathcal{G}_{2}, \mathcal{G}_{3}$, and $\mathcal{H}$ are as in Setup C. Then, for all but at most $10 \varepsilon_{3}^{1 / 64} m^{2}$ pairs $\left\{u, u^{\prime}\right\} \subset U_{1}$, the following is true:

$$
\left|\mathcal{H} \cap \mathcal{K}_{3}\left(\mathcal{H}\left(u, u^{\prime}\right)\left[\mathcal{G}_{3}\left(u, u^{\prime}\right)\right]\right)\right| \leq \frac{3}{2} d_{2}^{9} \tilde{d}_{3}^{9} d_{3}^{7} m^{3} .
$$


From this claim we deduce Claim 9.3 in Section 9 as follows.

Proof. Let $u \in U_{1}$ be an $\mathcal{H}$-nice vertex (cf. Definition 6.3), that is an $\mathcal{G}_{2}$-good vertex for which we have

(i) the link $\mathcal{H}(u)$ is $\left(2 \varepsilon_{3}^{1 / 2}, d_{3}, r\right)$-regular with respect to $\mathcal{G}_{2}\left[\mathcal{G}_{2}(u)\right]$,

(ii) $\mathcal{H}\left[\mathcal{G}_{2}(u)\right]$ is $\left(2 \varepsilon_{3}^{1 / 4}, d_{3}, r /\left(\varepsilon_{3}^{1 / 2} d_{2}^{-3}\right)\right)$-regular with respect to $\mathcal{G}_{2}\left[\mathcal{G}_{2}(u)\right]$, Also remind that the $\mathcal{G}_{2}$-goodness of $u$ implies

(iii) $\operatorname{deg}_{j}(u)=\left(d_{2} \pm \varepsilon_{2}\right) m$ for $j=2, \ldots, 5$,

(iv) the $(4,2)$-cylinder $\mathcal{G}_{2}\left[\mathcal{G}_{2}(u)\right]$ is $\left(\varepsilon_{2}^{1 / 2}, d_{2}\right)$-regular.

We apply Claim A.1 with $\mathcal{G}_{2}^{\prime}=\mathcal{G}_{2}\left[\mathcal{G}_{2}(u)\right], \mathcal{G}_{3}^{\prime}=\mathcal{H}(u)$, and $\mathcal{H}^{\prime}=\mathcal{H}\left[\mathcal{G}_{2}(u)\right]$. Observe that if we set $\varepsilon_{2}^{\prime}=\varepsilon_{2}^{1 / 2}, \varepsilon_{3}^{\prime}=2 \varepsilon_{3}^{1 / 4}, m^{\prime}=\left(d_{2} \pm \varepsilon_{2}\right) m, d_{2}^{\prime}=d_{2}$, $\tilde{d}_{3}^{\prime}=d_{3}^{\prime}=d_{3}$, and $r^{\prime}=r /\left(\delta_{3}^{1 / 2} d_{2}^{-3}\right)$, then (i)-(iv) verify conditions of the Setup C. More precisely,

- (iv) verifies that $\mathcal{G}_{2}^{\prime}$ is $\left(\varepsilon_{2}^{\prime}, d_{2}^{\prime}\right)$-regular,

- (i) verifies that $\mathcal{G}_{3}^{\prime}$ is $\left(\varepsilon_{3}^{\prime}, \tilde{d}_{3}^{\prime}, r^{\prime}\right)$-regular with respect to $\mathcal{G}_{2}^{\prime}$, and

- (ii) verifies that $\mathcal{H}^{\prime}$ is $\left(\varepsilon_{3}^{\prime}, d_{3}^{\prime}, r^{\prime}\right)$-regular with respect to $\mathcal{G}_{2}^{\prime}$.

By Claim A.1, all but $10\left(\varepsilon_{3}^{\prime}\right)^{1 / 64}\left(m^{\prime}\right)^{2}$ pairs $\left\{v, v^{\prime}\right\} \subset N_{\mathcal{G}_{2}, 2}(u)$ satisfy

$$
\left|\mathcal{H}^{\prime} \cap \mathcal{K}_{3}\left(\mathcal{H}^{\prime}\left(v, v^{\prime}\right)\left[\mathcal{G}_{3}^{\prime}\left(v, v^{\prime}\right)\right]\right)\right| \leq \frac{3}{2}\left(d_{2}^{\prime}\right)^{9}\left(\tilde{d}_{3}^{\prime}\right)^{9}\left(d_{3}^{\prime}\right)^{7}\left(m^{\prime}\right)^{3} .
$$

This concludes the proof because

- $\mathcal{H}^{\prime} \cap \mathcal{K}_{3}\left(\mathcal{H}^{\prime}\left(v, v^{\prime}\right)\left[\mathcal{G}_{3}^{\prime}\left(v, v^{\prime}\right)\right]\right)=\mathcal{H} \cap \mathcal{K}_{3}\left(\mathcal{H}\left(u, v, v^{\prime}\right)\left[\mathcal{H}(u)\left(v, v^{\prime}\right)\right]\right)$,

- $10\left(\varepsilon_{3}^{\prime}\right)^{1 / 64}\left(m^{\prime}\right)^{2} \leq 20 \varepsilon_{3}^{1 / 256} d_{2}^{2} m^{2}$, and

- $(3 / 2)\left(d_{2}^{\prime}\right)^{9}\left(\tilde{d}_{3}^{\prime}\right)^{9}\left(d_{3}^{\prime}\right)^{7}\left(m^{\prime}\right)^{3} \leq 2 d_{2}^{12} d_{3}^{16} m^{3}$.

Here we used the definitions of $\varepsilon_{2}^{\prime}, \varepsilon_{3}^{\prime}, m^{\prime}$, and $\varepsilon_{2} \ll d_{2}$.

To prove Claim A.1, we start with some technical observations. The first one is a consequence of Fact 5.6 and shows that almost all pairs have approximately the same joint degree in $\mathcal{G}_{3}$.

Fact A.2. For all but at most $10(s-1) \varepsilon_{3}^{1 / 8} m^{2}$ pairs $\left\{u, u^{\prime}\right\} \subset U_{1}$ we have:

$$
\operatorname{deg}_{\mathcal{G}_{3}, j}\left(u, u^{\prime}\right)=\left(1 \pm \varepsilon_{3}^{1 / 8}\right)^{2} d_{2}^{2} \tilde{d}_{3}^{2} m
$$

for every $j \in[s] \backslash\{1\}$. Consequently, $\mathcal{G}_{2}\left[\mathcal{G}_{3}\left(u, u^{\prime}\right)\right]$ is $\left(\varepsilon_{2}^{1 / 2}, d_{2}\right)$-regular.

The second fact follows from Claim 6.6 and shows that the restriction of $\mathcal{H}$ to the joint neighborhood of almost all pairs of vertices inherits regularity.

Fact A.3. Set $r^{\prime}=r /\left(\varepsilon_{3}^{1 / 2} d_{2}^{-3}\right)\left(2 \varepsilon_{3}^{1 / 8} d_{2}^{-3}\right)$. Then,

(i) the pair $\left\{u, u^{\prime}\right\}$ is good, and

(ii) the restriction $\mathcal{H}\left[\mathcal{G}_{2}\left(u, u^{\prime}\right)\right]$ is $\left(4 \varepsilon_{3}^{1 / 16}, d_{3}, r^{\prime}\right)$-regular with respect to $\mathcal{G}_{2}\left[\mathcal{G}_{2}\left(u, u^{\prime}\right)\right]$

for all but at most $5\left(\begin{array}{c}s-1 \\ 3\end{array}\right) \varepsilon_{3}^{1 / 2} m^{2}$ pairs $\left\{u, u^{\prime}\right\} \subset U_{1}$. 
The last fact shows that the $(s-1,2)$-cylinder $\mathcal{G}_{3}\left[\mathcal{G}_{3}\left(u, u^{\prime}\right)\right] \cap \mathcal{H}\left(u, u^{\prime}\right)$ is regular with respect to $\mathcal{G}_{2}\left[\mathcal{G}_{3}\left(u, u^{\prime}\right)\right]$ for almost all pairs $\left\{u, u^{\prime}\right\} \subset U_{1}$.

Fact A.4. For all but at most $3\left(\begin{array}{c}s-1 \\ 2\end{array}\right) \varepsilon_{3}^{1 / 64} m^{2}$ pairs of $\mathcal{G}_{2}$-good vertices $\left\{u, u^{\prime}\right\} \subset$ $U_{1}$ the following statements hold.

(a)

$$
\operatorname{deg}_{\mathcal{G}_{3}, j}\left(u, u^{\prime}\right)=\left(1 \pm \varepsilon_{3}^{1 / 8}\right)^{2} d_{2}^{2} \tilde{d}_{3}^{2} m,
$$

for every $j \in[s] \backslash\{1\}$,

(b) $\mathcal{G}_{2}\left[\mathcal{G}_{3}\left(u, u^{\prime}\right)\right]$ is $\left(\varepsilon_{2}^{1 / 2}, d_{2}\right)$-regular, and

(c) $\mathcal{G}_{3}\left[\mathcal{G}_{3}\left(u, u^{\prime}\right)\right] \cap \mathcal{H}\left(u, u^{\prime}\right)$ is $\left(\varepsilon_{3}^{1 / 64}, \tilde{d}_{3} d_{3}^{2}, r^{\prime}\right)$-regular with respect to $\mathcal{G}_{2}\left[\mathcal{G}_{3}\left(u, u^{\prime}\right)\right]$, where $r^{\prime}=r /\left(\varepsilon_{3}^{1 / 2} d_{2}^{-2}\right)\left(\varepsilon_{3}^{1 / 16} d_{2}^{-2}\right)$.

Parts (a) and (b) follows from Facts A.2. The proof of (c) is analogous to the proof of Claim 6.5 and consist of three steps.

i) the $(s-1,2)$-cylinder $\mathcal{G}_{3}\left[\mathcal{G}_{3}(u)\right] \cap \mathcal{H}(u)$ is $\left(\varepsilon_{3}^{1 / 8}, d_{3} \tilde{d}_{3}, r^{\prime}\right)$-regular with respect to $\mathcal{G}_{2}\left[\mathcal{G}_{3}(u)\right]$ for almost all $\mathcal{G}_{2}$-good vertices $u \in U_{1}$ (cf. Claim $7.3)$.

ii) the $(s-1,2)$-cylinder $\mathcal{G}_{3}\left[\mathcal{G}_{3}(u) \cap \mathcal{G}_{2}\left(u^{\prime}\right)\right] \cap \mathcal{H}(u)$ is $\left(2 \varepsilon_{3}^{1 / 32}, d_{3} \tilde{d}_{3}, r^{\prime}\right)$ regular with respect to $\mathcal{G}_{2}\left[\mathcal{G}_{3}(u) \cap \mathcal{G}_{2}\left(u^{\prime}\right)\right]$ for almost all pairs of $\mathcal{G}_{2^{-}}$ good vertices $\left\{u, u^{\prime}\right\} \subset U_{1}$.

iii) $\mathcal{G}_{3}\left[\mathcal{G}_{3}\left(u, u^{\prime}\right)\right] \cap \mathcal{H}\left(u, u^{\prime}\right)$ is $\left(\varepsilon_{3}^{1 / 64}, \tilde{d}_{3} d_{3}^{2}, r^{\prime}\right)$-regular with respect to $\mathcal{G}_{2}\left[\mathcal{G}_{3}\left(u, u^{\prime}\right)\right]$ for almost all pairs of $\mathcal{G}_{2}$-good vertices $\left\{u, u^{\prime}\right\} \subset U_{1}$.

A counterexample to step ii) (step iii), respectively) would yield an counterexample to step i) (regularity of $\mathcal{H}$ with respect to $\mathcal{G}_{2}$, respectively). For details we refer the interested reader to [Sk00]. Now we are ready to prove Claim A.1.

Proof of Claim A.1. Set $r^{\prime}=r /\left(\varepsilon_{3}^{1 / 2} d_{2}^{-3}\right)\left(\varepsilon_{3}^{1 / 16} d_{2}^{-3}\right)$ and let $\left\{u, u^{\prime}\right\}$ be a pair of vertices such that

(i) $\left\{u, u^{\prime}\right\}$ is a good pair,

(ii) the restriction of $\mathcal{H}$ to $\mathcal{G}_{2}\left(u, u^{\prime}\right)$ is $\left(2 \varepsilon_{3}^{1 / 16}, d_{3}, r^{\prime}\right)$-regular with respect to $\mathcal{G}_{2}\left[\mathcal{G}_{2}\left(u, u^{\prime}\right)\right]$,

(iii) $\operatorname{deg}_{\mathcal{G}_{3}, j}\left(u, u^{\prime}\right)=\left(1 \pm \varepsilon_{3}^{1 / 8}\right)^{2} d_{2}^{2} \tilde{d}_{3}^{2} m$ for every $j \in[s] \backslash\{1\}, \mathcal{G}_{2}\left[\mathcal{G}_{3}\left(u, u^{\prime}\right)\right]$ is $\left(\varepsilon_{2}^{1 / 2}, d_{2}\right)$-regular, and

(iv) $\mathcal{G}_{3}\left[\mathcal{G}_{3}\left(u, u^{\prime}\right)\right] \cap \mathcal{H}\left(u, u^{\prime}\right)$ is $\left(\varepsilon_{3}^{1 / 64}, \tilde{d}_{3} d_{3}^{2}, r^{\prime}\right)$-regular with respect to $\mathcal{G}_{2}\left[\mathcal{G}_{3}\left(u, u^{\prime}\right)\right]$.

It follows from Fact A.3 that all but at most $5 \varepsilon_{3}^{1 / 2} m^{2}$ pairs $\left\{u, u^{\prime}\right\}$ satisfy (i) and (ii) (note that $r^{\prime} \leq r /\left(\varepsilon_{3}^{1 / 2} d_{2}^{-3}\right)\left(2 \varepsilon_{3}^{1 / 8} d_{2}^{-3}\right)$ ).

Furthermore, Fact A.4 impies that all but at most $9 \varepsilon_{3}^{1 / 64} \mathrm{~m}^{2}$ good pairs $\left\{u, u^{\prime}\right\}$ satisfy (iii) and (iv) (note that in this case $r^{\prime} \leq r /\left(\varepsilon_{3}^{1 / 2} d_{2}^{-2}\right)\left(\varepsilon_{3}^{1 / 16} d_{2}^{-2}\right)$ ).

We define two $(3,2)$-cylinders $\mathcal{G}_{1}^{\prime}, \mathcal{G}_{2}^{\prime}$ by $\mathcal{G}_{1}^{\prime}=\mathcal{G}_{2}\left[\mathcal{G}_{3}\left(u, u^{\prime}\right)\right]$ and $\mathcal{G}_{2}^{\prime}=$ $\mathcal{G}_{3}\left[\mathcal{G}_{3}\left(u, u^{\prime}\right)\right] \cap \mathcal{H}\left(u, u^{\prime}\right)$. 
Then, $\mathcal{G}_{1}^{\prime}$ and $\mathcal{G}_{2}^{\prime}$ satisfy the assumptions of the 2-graphs Lemma. More precisely, setting $\varepsilon_{1}^{\prime}=\varepsilon_{2}^{1 / 2}, \varepsilon_{2}^{\prime}=\varepsilon_{3}^{1 / 64}, d_{1}^{\prime}=d_{2}, d_{2}^{\prime}=\tilde{d}_{3} d_{3}^{2}$, and $m^{\prime}=$ $\left(1 \pm \varepsilon_{3}^{1 / 8}\right)^{2} d_{2}^{2} \tilde{d}_{3}^{2} m$, we have that $\mathcal{G}_{1}^{\prime}$ is $\left(\varepsilon_{1}^{\prime}, d_{1}^{\prime}\right)$-regular and $\mathcal{G}_{2}^{\prime}$ is $\left(\varepsilon_{2}^{\prime}, d_{2}^{\prime}, r^{\prime}\right)$ regular with respect to $\mathcal{G}_{1}^{\prime}$ (cf. (iii) and (iv)).

We apply the 2-graphs Lemma and obtain the following

$$
\left|\mathcal{K}_{3}\left(\mathcal{G}_{3}\left[\mathcal{G}_{3}\left(u, u^{\prime}\right)\right] \cap \mathcal{H}\left(u, u^{\prime}\right)\right)\right|=\left|\mathcal{K}_{3}\left(\mathcal{G}_{2}^{\prime}\right)\right| \geq\left(1-\varepsilon_{2}^{\prime 1 / 64}\right)^{2}\left(d_{1}^{\prime} d_{2}^{\prime}\right)^{3}\left(m^{\prime}\right)^{3}
$$

Then we use the definitions of $\varepsilon_{1}^{\prime}, \varepsilon_{2}^{\prime}$ and $m^{\prime}$ and assumption $\varepsilon_{2} \ll \varepsilon_{3} \ll$ $d_{3} \leq 1$ to obtain

$$
\begin{aligned}
\mid \mathcal{K}_{3}\left(\mathcal{G}_{3}\left[\mathcal{G}_{3}\left(u, u^{\prime}\right)\right]\right. & \left.\cap \mathcal{H}\left(u, u^{\prime}\right)\right) \mid \\
\geq & \left(1-\varepsilon_{2}^{1 / 4096}\right) d_{2}^{3} \tilde{d}_{3}^{3} d_{3}^{6}\left(\left(1-\varepsilon_{3}^{1 / 8}\right)^{2} d_{2}^{2} \tilde{d}_{3}^{2} m\right)^{3} \geq \frac{3}{4} d_{2}^{9} \tilde{d}_{3}^{9} d_{3}^{6} m^{3} .
\end{aligned}
$$

In a similar fashion we get

$$
\left|\mathcal{K}_{3}\left(\mathcal{G}_{3}\left[\mathcal{G}_{3}\left(u, u^{\prime}\right)\right] \cap \mathcal{H}\left(u, u^{\prime}\right)\right)\right| \leq \frac{5}{4} d_{2}^{9} \tilde{d}_{3}^{9} d_{3}^{6} m^{3} .
$$

Since the pair $\left\{u, u^{\prime}\right\}$ is $\mathcal{G}_{2}$-good (cf. (i)), the $(3,2)$-cylinder $\mathcal{G}_{2}\left[\mathcal{G}_{2}\left(u, u^{\prime}\right)\right]$ is $\left(\varepsilon_{2}^{1 / 2}, d_{2}\right)$-regular. It follows from Corollary 4.6 that

$$
\left|\mathcal{K}_{3}\left(\mathcal{G}_{2}\left[\mathcal{G}_{2}\left(u, u^{\prime}\right)\right]\right)\right| \leq(5 / 4) d_{2}^{3}\left(d_{2}+\varepsilon_{2}^{1 / 2}\right)^{6} m^{3} \leq 2 d_{2}^{9} m^{3} .
$$

Since $4 \varepsilon_{3}^{1 / 16} \times 2 d_{2}^{9} \leq(3 / 4) d_{2}^{9} \tilde{d}_{3}^{9} d_{3}^{6}$ (because of $\varepsilon_{3} \ll d_{3}$ and $\varepsilon_{3} \ll \tilde{d}_{3}$ ), we obtain

$$
\left|\mathcal{K}_{3}\left(\mathcal{G}_{3}\left[\mathcal{G}_{3}\left(u, u^{\prime}\right)\right] \cap \mathcal{H}\left(u, u^{\prime}\right)\right)\right| \geq 4 \varepsilon_{3}^{1 / 16}\left|\mathcal{K}_{3}\left(\mathcal{G}_{2}\left[\mathcal{G}_{2}\left(u, u^{\prime}\right)\right]\right)\right| .
$$

We apply the $\left(4 \varepsilon_{3}^{1 / 16}, d_{3}, r^{\prime}\right)$-regularity of $\mathcal{H}\left[\mathcal{G}_{2}\left(u, u^{\prime}\right)\right]$ with respect to $\mathcal{G}_{2}\left[\mathcal{G}_{2}\left(u, u^{\prime}\right)\right]$ and obtain

$$
\begin{aligned}
&\left|\mathcal{H} \cap \mathcal{K}_{3}\left(\mathcal{H}\left(u, u^{\prime}\right)\left[\mathcal{G}_{3}\left(u, u^{\prime}\right)\right]\right)\right| \\
& \leq\left|\mathcal{H} \cap \mathcal{K}_{3}\left(\mathcal{G}_{3}\left[\mathcal{G}_{3}\left(u, u^{\prime}\right)\right] \cap \mathcal{H}\left(u, u^{\prime}\right)\right)\right| \\
& \leq\left(d_{3}+2 \varepsilon_{3}^{1 / 16}\right)\left|\mathcal{K}_{3}\left(\mathcal{G}_{3}\left[\mathcal{G}_{3}\left(u, u^{\prime}\right)\right] \cap \mathcal{H}\left(u, u^{\prime}\right)\right)\right| \\
& \stackrel{(\text { A.3) }}{\leq}\left(d_{3}+4 \varepsilon_{3}^{1 / 16}\right) \frac{5}{4} d_{2}^{9} \tilde{d}_{3}^{9} d_{3}^{6} m^{3} \leq \frac{3}{2} d_{2}^{9} \tilde{d}_{3}^{9} d_{3}^{7} m^{3} .
\end{aligned}
$$

Emory University, Atlanta, GA, 30322, USA

E-mail address: rodl@mathcs.emory.edu

University of Illinois at Urbana-Champaign, 1409 West Green Street, URBAna, IL, 61801, USA and Instituto de Matemática e Estatística, Universidade DE SÃo Paulo, Rua do Matão 1010, 05508-900 SÃo Paulo, Brazil

E-mail address: jozef@member.ams.org 